WEIGHT STIGMA AT A UNIVERSITY:

\title{
A STUDY OF POLICY, MEDIA, AND STUDENT EXPERIENCES WITH IMPLICATIONS FOR IMPROVING WEIGHT-INCLUSIVITY
}

A Dissertation
presented to
the Faculty of the Graduate School
at the University of Missouri-Columbia
In Partial Fulfillment
of the Requirements for the Degree
Doctor of Education
by
HILLARY L. ROBERTS
Dr. Cynthia MacGregor, Dissertation Supervisor
MAY 2021


(C) Copyright by Hillary L. Roberts 2021

All Rights Reserved 
The undersigned, appointed by the dean of the Graduate School, have examined the dissertation entitled

WEIGHT STIGMA AT A UNIVERSITY:

A STUDY OF POLICY, MEDIA, AND STUDENT EXPERIENCES WITH IMPLICATIONS FOR IMPROVING WEIGHT-INCLUSIVITY

presented by Hillary L. Roberts, a candidate for the degree of doctor of education, and hereby certify that, in their opinion, it is worthy of acceptance.

Dr. Cynthia MacGregor

Dr. Denise Baumann

Dr. Jeffrey Cornelius-White

Dr. Michael Hudson 


\section{DEDICATION}

To my husband, Brandon: Your encouragement and support mean the world to me. Thank you for always being there.

To my daughter, Clara: Your curiosity and love for learning are contagious. These past few months you have pretended to be working on your dissertation. Little do you know how much you inspire and motivate me to be the best person I can be.

To my daughter, Mara: I will meet you in a few weeks and look forward to learning from you just as I have from your sister. 


\section{ACKNOWLEDGEMENTS}

I would like to thank my dissertation advisor, Dr. Cynthia MacGregor. Thank you for the unconditional support and guidance you have provided me throughout the program and the dissertation process. Additionally, I would like to thank my dissertation committee: Dr. Denise Baumann, Dr. Jeffrey Cornelius-White, and Dr. Michael Hudson. Little did I know how lucky I was that each of you had different strengths in the research and writing process. Thank you for taking the time to guide me as a researcher and writer.

I would also like to thank my colleagues who helped give me the strength to keep going when the going was tough. Sarah Murray was always there with a coffee or special treat when I needed a pick-me-up before Wednesday night class. Thank you all!

Lastly, I would like to acknowledge the students in Cohort 11. We all learned so much together, from the complexity of the Wicked problem in Summer One at the University of Missouri-Columbia, to research design and analysis from our small group projects. I am thankful for the camaraderie, especially at the Missouri State University site. 


\section{TABLE OF CONTENTS}

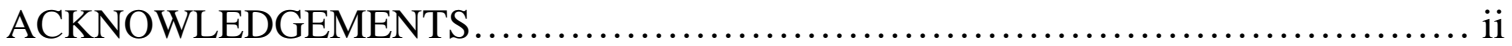

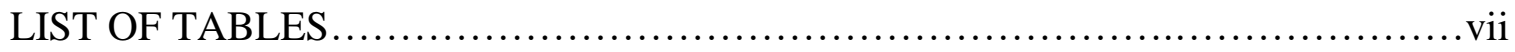

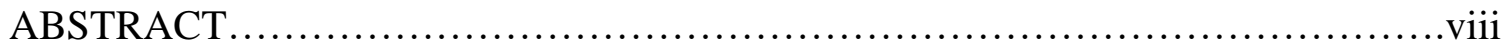

Section

1. INTRODUCTION TO DISSERTATION ..............................

Researcher Positionality Statement................................... 2

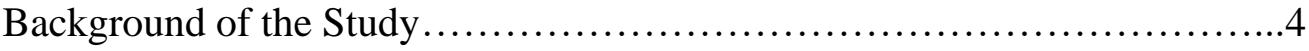

Statement of the Problem...............................................6

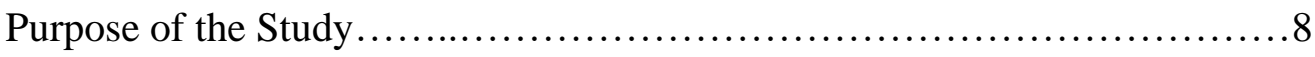

Research Questions..............................................9

Theoretical Framework........................................... 10

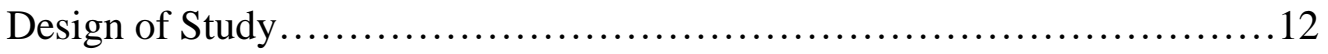

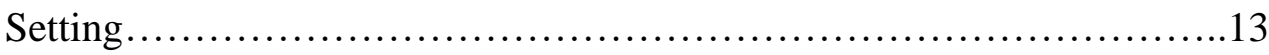

Participants................................................ 15

Data Collection Tools............................................17

Data Analysis.................................................20

Limitations, Assumptions, and Design Controls.......................23

Definitions of Key Terms.........................................24

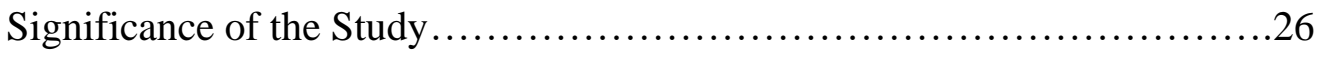

Summary ....................................................... 28

2. PRACTITIONER SETTING FOR THE STUDY .......................29

Introduction......................................................... 
History of Organization...................................................

Public Affairs Mission................................................... 30

Values..............................................................

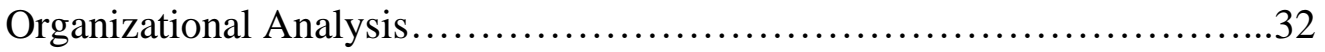

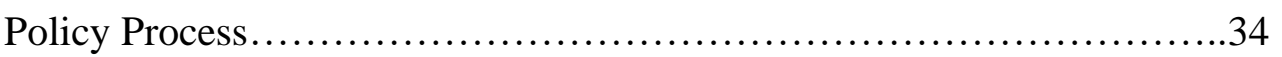

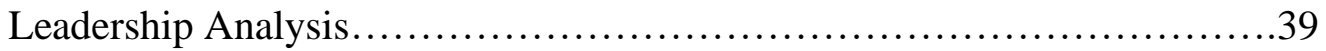

Implications for Research in the Practitioner Setting ......................42

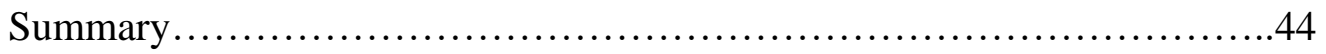

3. SCHOLARLY REVIEW FOR THE STUDY ...........................46

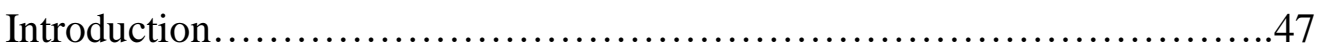

Health at Every Size Theoretical Model.................................48

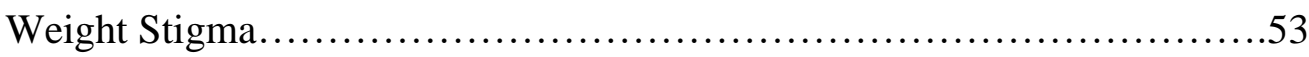

Higher Education.......................................................

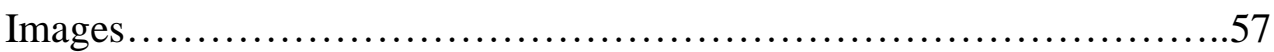

Summary of Weight Stigma Scholarly Review...........................59

Weight Discrimination and Policy.......................................60

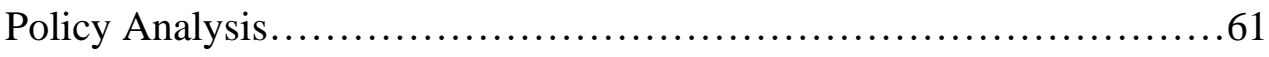

Summary of Weight Discrimination and Policy..........................64

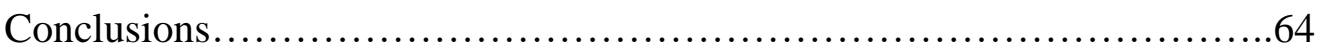

4. CONTRIBUTION TO PRACTICE ...................................66

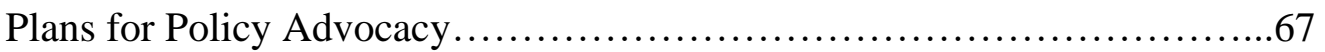

Executive Summary and Policy Brief...................................69 
5. CONTRIBUTION TO SCHOLARSHIP .............................. 74

Author's Note........................................................... 75

Abstract........................................................... 76

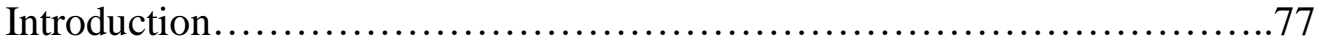

Method.......................................................... 80

Participants and Procedures........................................80

Measures........................................................... 81

Data Analytic Plan.............................................83

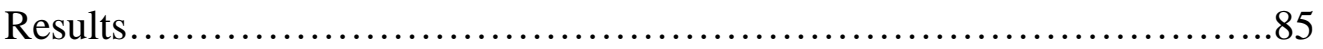

Student Experiences of University Body Messages....................85

University's Website Images.......................................88

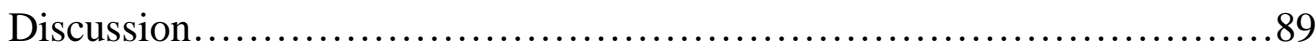

References.....................................................95

6. SCHOLARLY PRACTITIONER REFLECTION ....................... 100

Influence on Practice as an Educational Leader..........................101

Connectedness.................................................101

Resilience..................................................103

Influence of Practice as a Scholar...................................... 105

Conclusion....................................................107

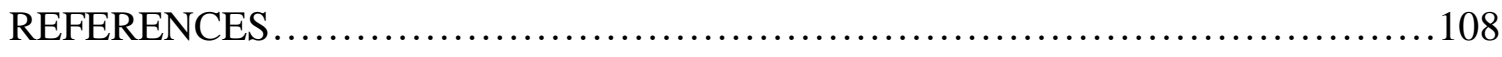

\section{APPENDICES}

A. Recruitment Post in Student Wellness Newsletter........................121

B. Research Participation Recruitment Email and Flyer................... 123 


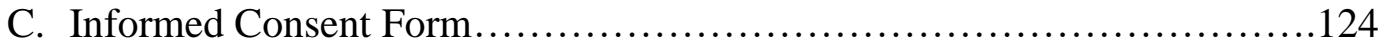

D. Screening Email Script............................................... 127

E. Sociodemographic Survey.......................................128

F. Photo-Elicitation Instructions....................................... 130

G. Photo-Elicitation Questionnaire..................................131

H. Photo-Elicitation Questionnaire Email................................132

I. Document Policy Analysis Protocol................................... 133

J. Messaging Analysis Protocol......................................134

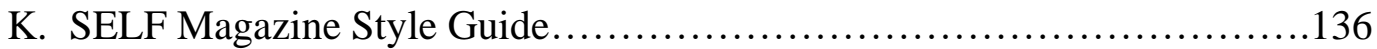

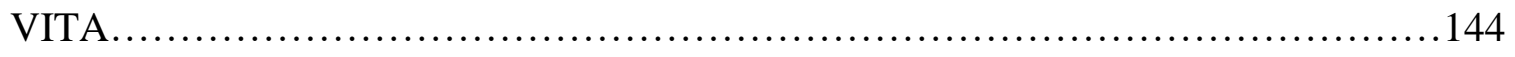




\section{LIST OF TABLES}

Table $\quad$ Page

1. Weight Inclusive Findings from Photo-Elicitation Questionnaires...............86

2. Weight Stigmatizing Findings from Photo-Elicitation Questionnaires...........87

3. Findings from Weight Inclusive Images of People Collected on University

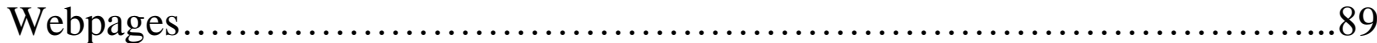




\title{
WEIGHT STIGMA AT A UNIVERSITY: \\ A STUDY OF POLICY, MEDIA, AND STUDENT EXPERIENCES WITH IMPLICATIONS FOR IMPROVING WEIGHT-INCLUSIVITY \\ Hillary L. Roberts \\ Dr. Cynthia MacGregor, Dissertation Supervisor
}

\begin{abstract}
Media images of and text concerning people who are overweight or obese frequently perpetuate weight stigma. Additionally, limited literature exists on policy and weight stigma. This qualitative study explored college student perspectives of body messaging at a public university, and investigated university policy and images of people used on university webpages for having weight inclusive or weight stigmatizing body messaging. Body messaging included images of people and text. The study engaged a sample of seven participants through photo-elicitation to collect body messages the university used in print materials and social media platforms. Participants then completed a questionnaire about each photo. Additionally, the researchers collected additional images of people from the university webpages. From the 27 participantgenerated photos, over a third did not have size diversity or were placed in nonprominent locations on campus. Almost three-fourths of the 65 images of people collected by the researchers from the university webpages, did not include size diversity. Based upon the research findings, recommendations are provided for university constituents on how to promote weight inclusive environments through policy, programs, and practice.
\end{abstract}

Keywords: images, media, messages, photo-elicitation, policy, weight stigma 
SECTION ONE:

INTRODUCTION TO DISSERTATION 


\section{Researcher Positionality Statement}

I am a small person. In grade school I was teased because of my body size and distinctively remember being called names such as shrimp. In addition, I entered puberty much later in my peers, not finishing developing and growing in stature until college. Regardless of growing up on a beef cattle farm where our meals primarily consisted of red meat, potatoes, and bread, I remained a small person.

I entered college as a first-generation college student having a love for the medical field and unsure of what health profession to pursue. That changed when I took an introductory nutrition course and found I loved learning about the gastrointestinal system. I declared the major of dietetics beginning my junior year and have now been a registered dietitian nutritionist for over 15 years. Unbeknownst to me at the time, I entered a profession where my body size continues to be judged. While I have experienced weight stigma, it does not compare to the weight stigma people who are overweight or obese face. I have what some call "thin privilege" and although people feel at liberty to comment on my weight, I do not have society telling me I am less of a person because of my weight.

In my career as a dietitian, I have provided nutrition counseling to numerous clients who had deep resentment of their body size. Although many of these clients were metabolically healthy, they were referred to me due to their body mass index. While a person's health cannot be defined by their body mass index, many clients believe this to be truth as it is what society tells them and reinforces. I now teach nutrition and dietetic students at a public university and use the Health at Every Size (HAES) paradigm as my guiding perspective. 
When thinking about a dissertation topic I knew I wanted a topic to help decrease weight stigma for people who are overweight or obese in addition to incorporating the HAES paradigm principles. Through reading the current literature I chose to focus on body messaging and the words and images of people used in this messaging. Although I am a small person and an outsider regarding body size, this research is close to my heart and it is my hope this study can make an impact in decreasing weight stigma within higher education. 


\section{Background of the Study}

Stigma toward people of different races, genders, and religions have been rejected by civilized society and these stigmas are challenged. Weight stigma on the other hand remains socially acceptable (Puhl \& Heuer, 2010). This is acceptable to such a great degree that health campaigns at times endorse using stigmatizing approaches toward people who are overweight or obese (Puhl, Luedicke, \& Peterson, 2013). Furthermore, the media perpetuates weight stigma by frequently depicting people who are overweight or obese in negative contexts (Heuer, McClure, \& Puhl, 2011).

It is important to note within the literature on weight stigma, the terms weight stigma and weight bias are used interchangeably. This aligns with concepts in Pescosolido and Martin's (2015) article, The Stigma Complex. In this article they discussed how within stigma research, terms targeting the same phenomena are often used interchangeably, and how stigma is now a "global referent category" (p. 5). According to Puhl, Moss-Racusin, Schwartz, and Brownell (2008), weight stigma or weight bias is the "negative weight-related attitudes and beliefs that are manifested by stereotypes, rejection and prejudice toward individuals because they are overweight or obese" (p. 347). In an editorial about weight stigma in the Lancet Public Health (2019), weight stigma and weight bias were defined as "the discrimination, stereotyping, and social exclusion based on a person's weight” (p. 168).

Within this study, the researcher will be using the term weight stigma. To avoid confusion, the term weight bias will not be used, except when referencing scholarly research that has used this term. The researcher proposes to use Pescosolido and Martin's (2015) definitions for the overarching concepts of stigma (i.e., stigma, prejudice, 
discrimination). However, since there is no defining distinction between weight stigma and weight bias in the literature, the researcher created and proposed definitions for these two terms to differentiate between the two (see Definition of Key Terms, p. 24).

While there is not a universal definition for weight stigma, the consequences of weight stigma have been well documented. These consequences are severe and include having a negative effect on the psychological and physical health of individuals who experience weight stigma (Gloor \& Puhl, 2016; Puhl \& Heuer, 2009). Examples of these negative effects are depression, lowered self-esteem, body image dissatisfaction, bingeeating behaviors, and reduced or avoidance of physical activity (Puhl \& Heuer, 2009). Additionally, negative stereotypes associated toward individuals who are overweight or obese are widely documented in research literature. These stereotypes contribute to weight stigma and include lazy, unintelligent, lack of self-discipline, and unmotivated (Puhl \& Brownell, 2001; Puhl \& Heuer, 2009).

According to Pescosolido and Martin (2015), a component of stigma is "experiencing status loss and discrimination" (p. 6), where the stigmatized have unjust treatment. For people who are overweight or obese, this is referred to as weight discrimination. Weight discrimination is the "unjust treatment of individuals because of their weight" (Ramos-Salas et al., 2017, p. 1323). The prevalence of self-reported perceived weight discrimination increased from $7.3 \%$ in $1995-1996$ to $12.2 \%$ in 2004 2006 (Andreyeva, Puhl, \& Brownell, 2008). This discrimination occurs across numerous settings including the workplace, higher education, healthcare facilities, and interpersonal relationships (Burmeister et al., 2016; Puhl \& Heuer, 2009; Puhl \& King, 2013). For example, weight discrimination has contributed to individuals not being promoted 
(Burmeister et al., 2016; Puhl \& Heuer, 2009), being disfavored in interviews for graduate school (Burmeister et al., 2016), and receiving negligent healthcare treatment (Burmeister et al., 2016).

Regardless of research documenting weight discrimination and the negative effects this discrimination has on the individuals who experience it, in the United States only the state of Michigan and six municipalities have legislation in place prohibiting weight discrimination (Pearl, Puhl, \& Dovidio, 2017). In addition, higher education institutions have policies related to diversity and inclusiveness, yet within these policies, weight discrimination is seldom addressed. The exception is higher education institutions located in Michigan, due to Michigan law prohibiting weight discrimination in education (Michigan Department of Civil Rights, 2019).

Stigmatizing beliefs and attitudes are maintained and reinforced with exposure to negative messages toward people who are overweight or obese. Within higher education, there is sparse research on how students experience weight stigma, especially through institutional messages. Cameron (2016), a critical obesity scholar, stated, "the importance of identifying strategies for reducing obesity stigma in higher education...cannot be understated, given the role higher education can play in forming (or changing) attitudes and beliefs about obesity, bodies, and health" (p. 120).

\section{Statement of the Problem}

Weight stigma is pervasive in North American society (Puhl \& Heuer, 2010; Ramos-Salas et al., 2017). Within the context of education, research has found young adults who are obese are less likely to attain a college degree (Fowler-Brown, Ngo, Phillips, \& Wee, 2010), especially females (Benson, von Hippel, Lynch, 2017; Crosnoe, 
2007). Crosnoe (2007) used data from the National Longitudinal Study of Adolescent Health and reported females who are obese are 50\% less likely to attend college than females who are not obese. It is speculated weight stigma contributes to these lower rates of achievement. Puhl and Brownell (2006) discovered 32\% of adult females $(n=2,449)$ had experienced weight stigma from a teacher or professor, with $21 \%$ experiencing this stigma from a teacher or professor more than once. Male students also experience weight stigma within education. In a sample size of 111 males, $27 \%$ had experienced weight stigma from an educator (Puhl \& Brownell, 2006).

Research on how students experience weight stigma at higher education institutions, especially through institutional messages, is limited. The only study found on this topic was by Stevens (2018). She found considerable amounts of weight stigma in her exploratory study of qualitative data collected from 14 students at two midwestern state universities. Stevens (2018) stated, "fat students are bombarded with antiobesity information around campus" (p. 136). The consistent rhetoric to lose weight contributes to individuals having lower self-esteem and poor body image (Stevens, 2018; Wellman, Araiza, Newell, \& McCoy, 2018). Although Stevens's study did not include document collection, her participants noted that documents on campus (e.g., campus newspaper, signs in bathroom stalls, materials at student health center) triggered feelings of weight stigma.

In the limited studies on the consequences of weight stigma within higher education, research has suggested weight stigmatizing experiences from college instructors may contribute to decreased academic self-efficacy in students (Aimé, Villatte, Cyr, \& Marcotte, 2017). Students who were overweight or obese had reduced 
self-efficacy and reduced class attendance than students who were not overweight or obese (Aimé et al., 2017).

Regarding policy, there is currently no national weight anti-discrimination legislation to protect individuals from this type of discrimination. Although there is public support for this legislation (Puhl, Suh, \& Li, 2016), only the state of Michigan and six municipalities in the United States have enacted law to prohibit weight discrimination (Pearl, Puhl, \& Dovidio, 2017). To reduce weight stigma, several scholars have proposed to implement weight-inclusive policies (Pearl, Puhl, \& Dovidio, 2017; Puhl, Suh, \& Li, 2016; O’Reilly \& Sixsmith, 2012; Ramos-Salas et al., 2017). These policies would not protect individuals from weight discrimination; however, the policies would contribute to promoting weight inclusive environments which helps to decrease weight stigma (O’Reilly \& Sixsmith, 2012).

There is sparse research on the sources contributing to weight stigma in higher education, in addition to higher education policies that promote weight inclusive environments. With $37 \%$ of college students being overweight or obese (American College Health Association, 2020), further research is needed to investigate how students experience weight stigma within higher education. Furthermore, research is needed to investigate weight stigmatizing and weight inclusive language in higher education policies.

\section{Purpose of the Study}

The purpose of this research study was twofold. First, the researcher investigated policies of a midwestern, master's comprehensive public university and images of people used on the university's website for weight inclusive and weight stigmatizing body 
messages. Body messages referred to images of people, in addition to written content about body shape, size, and health. The policy analysis provided knowledge on how policies contributed to, or detracted from, implementing a weight inclusive environment in the university setting. While the analysis of images of people used on the university's website provided knowledge on how images contributed to, or detracted from, providing a weight inclusive environment in the university setting.

In addition, through photo-elicitation and a questionnaire, the researcher explored college student experiences of body messaging at a midwestern, master's comprehensive public university, and whether the messages used weight inclusive text and images of people, or weight stigmatizing text and images of people. Weight stigma remains socially acceptable. The researcher hopes this study will increase awareness of weight stigma and contribute knowledge on how body messaging and policies of higher education institutions can be used to promote weight inclusive environments to aid in decreasing weight stigma for all students.

\section{Research Questions}

The research study was guided by four research questions:

1. What policies at a public university include weight inclusive body messages, if any?

2. What policies at a public university include weight stigmatizing body messages, if any?

3. What weight inclusive body messages do college students experience at a public university?

4. What weight stigmatizing body messages do college students experience at a public university? 


\section{Theoretical Framework}

The theoretical model used to help provide a structure for this study was the Health at Every Size (HAES®) paradigm (see Footnote 1). HAES is a weight inclusive versus weight normative approach to policy and individual decision making, grounded in a social justice framework (Association for Size Diversity and Health [ASDAH], 2018). The weight inclusive approach emphasizes "viewing health and well-being as multifaceted while directing efforts toward improving health access and reducing weight stigma" (Tylka et al., 2014, p. 1). In contrast, the weight normative approach emphasizes, "weight and weight loss when defining health and well-being" (Tylka et al., 2014, p. 1).

Focusing on how to improve healthy living versus focusing on weight is the HAES model's foremost foundation (ASDAH, 2018). The HAES principles, as established by the Association for Size Diversity and Health (2018) are (a) weight inclusivity, (b) health enhancement, (c) respectful care, (d) eating for well-being, and (e) life-enhancing movement.

The five HAES principles encompassed the spirit of this study and were used to help guide the research methods including data analysis. Definitions for these principles are:

1. Weight inclusivity: Accept and respect the inherent diversity of body shapes and sizes and reject the idealizing or pathologizing of specific weights.

2. Health enhancement: Support health policies that improve and equalize access to information and services, and personal practices that improve human well-

\footnotetext{
${ }^{1}$ Health at Every Size and HAES are registered trademarks of the Association for Size Diversity and Health and used with permission.
} 
being, including attention to individual physical, economic, social, spiritual, emotional, and other needs.

3. Respectful care: Acknowledge our biases, and work to end weight discrimination, weight stigma, and weight bias. Provide information and services from an understanding that socioeconomic status, race, gender, sexual orientation, age, and other identities impact weight stigma, and support environments that address these inequities.

4. Eating for well-being: Promote flexible, individualized eating based on hunger, satiety, nutritional needs, and pleasure, rather than any externally regulated eating plan focused on weight control.

5. Life-enhancing movement: Support physical activities that allow people of all sizes, abilities, and interests to engage in enjoyable movement, to the degree that they choose. (ASDAH, 2018)

Principle one, weight inclusivity, and principle three, respectful care, aligned with the study's research questions. Documenting and analyzing university body messages and policy for weight inclusive and weight stigmatizing discourse provided data on whether or not the university's messages and policies portrayed weight inclusivity. In addition, this study provided data to aid in illuminating whether or not the university had actions contributing to weight stigmatizing environments. The remaining three principles, health enhancement, eating for well-being, and life-enhancing movement, contributed to the development of the data analysis protocols.

There is conclusive research showing the benefits of weight-inclusive approaches such as the HAES paradigm (Bacon \& Aphramor, 2011; Mensinger, Tylka, \& Calamari, 
2018; Tylka et al., 2014). Weight inclusive approaches have shown to increase size acceptance and self-esteem for participants who were overweight or obese (Bacon \& Aphramor, 2011). In addition, these approaches aided in reducing healthcare professionals weight bias thus decreasing internalization of weight stigma by their clients (Mensinger et al., 2018; Tylka et al., 2014). Research about weight inclusive messaging and policies in higher education are limited. In this study the researcher used photoelicitation to understand college student experiences of weight stigma in addition to document collection, screening university body messages and policies for weight inclusive versus weight stigmatizing content.

\section{Design of the Study}

The researcher was interested in investigating what weight inclusive body messages and weight stigmatizing body messages, if any, were included in university policy and through images of people used on the university's website. Additionally, the researcher sought to understand college students' perceptions of whether or not body messages produced and used by a midwestern university portrayed weight inclusiveness. This study utilized two research methodologies: (a) qualitative content analysis (Kuckartz, 2014) for all four research questions, and (b) phenomenology (Creswell, 2013) for research questions three and four. In addition, the research was conducted in a "bounded system" (Merriam \& Tisdell, 2016, p. 37), classifying the research as a case study.

Qualitative content analysis (Kuckartz, 2014) was used to analyze university archival records (i.e., policies) and images of people used on the university website for weight inclusive and weight stigmatizing content. Additionally, the study used 
phenomenological research design as suggested by Creswell (2013), due to having sought the lived experiences of students. The phenomena explored was weight inclusivity and weight stigma. The objective was to understand how participants experienced weight inclusivity and weight stigma through university body messages, understanding the "essence" (Creswell, 2013, p. 79) of their experiences, and how "experiencing something is transformed into consciousness" (Merriam \& Tisdell, 2016, p. 26).

These experiences were sought using photo-elicitation and a photo-elicitation questionnaire. The term, photo-elicitation, was named by John Collier in 1957 (Harper, 2002). Collier and Collier (1986) stated, "photographs can be communication bridges between strangers that can become pathways into unfamiliar, unforeseen environments and subjects" (p. 99). By having student participants take photos, this became a studentcentered versus researcher-centered approach of gaining student insights into body messaging at a university. Results from the qualitative content analysis were then converged with the results from the phenomenological analysis. This convergence of results increased the rigor, or trustworthiness, of the study (Ryan, Coughlan, \& Cronin, 2007; Tracy, 2010).

\section{Setting}

This study was conducted at a midwestern, master's comprehensive public university. While this study site is a public university system composed of four campuses, only one campus was used for this study. In 2020, this university had 24,163 students enrolled on its primary campus, with self-reported gender being $60 \%$ female and 40\% male (MSU Office of Institutional Research, 2020a). Of these students, 82.8\% reported they had in-state residency, and $63.1 \%$ were 21 years old or younger (MSU 
Office of Institutional Research, 2020a). In addition, student self-reported ethnicities were, 79.7\% White, 3.5\% African American, 4.2\% Hispanic, 2.0\% Asian, 0.4\% American Indian/Alaska Native, $0.1 \%$ Native Hawaiian/Pacific Islander, and the remaining $10.2 \%$ reported either two or more races, all races and ethnicities, or no race provided MSU Office of Institutional Research, 2020a).

In 1995, the state legislature approved designating the study site with a mission in public affairs (MSU, 2018a). Within this mission are tenants referred to as the three pillars of public affairs: (a) ethical leadership, (b) community engagement, and (c) cultural competence (MSU, 2018a). The vision of the university is to "be the university of choice to develop successful students who excel academically and in ethical leadership, cultural competence, and community engagement” (MSU, 2018b, Vision section, para. 2).

Values of the university include inclusiveness, promoting an environment of respect for all individuals, and continuous improvement in programs and policies (MSU, 2018b). The university categorizes inclusiveness with being fair, equitable, and valuing social justice. These items are defined as "the recognition that each person possesses not just one, but multiple identities; and the celebration of the similarities, as well as the differences, of our civility, trust and collaboration" (MSU, 2018b, Values section, para. $3)$.

In addition, this university has a Division for Diversity and Inclusion (DDI). DDI's four areas of emphasis are (a) access, success, and equity; (b) learning and development; (c) campus climate; and (d) institutional leadership and commitment (MSU, 2021a). The vision of DDI includes seeking to "build a community that values 
the inclusion of diversity in accordance with the Long-Range Plan, our annual Action Plan(s), and the Diversity \& Inclusion Implementation Strategy" (MSU, 2021a, Diversity and Inclusion Vision section, para. 1). The Diversity and Inclusion Implementation Strategy was created in January of 2018. This strategy provided a list of items for university administrators, faculty, and staff to use to help guide the development of “particular programs, initiatives, goals, and action items if and when strengths, weaknesses, opportunities and threats are analyzed and resources are made available" (MSU, 2021b Diversity and Inclusion Strategy, para. 2).

\section{Participants}

Various participant recruitment strategies were attempted. The researcher sought to follow purposeful sampling to gain rich data from a small sample size (Creswell, 2014; Gray, 2018; Merriam \& Tisdell, 2016). The type of purposeful sampling strategy she used was criterion sampling (Gray, 2018). One criterion was that participants were university students, with students defined as anyone currently enrolled in any universitylevel course who is 18 years of age or older. In addition, criterion included participants to self-report their body size status as overweight or obese.

Due to coronavirus disease 2019 (COVID-19), the researcher was not permitted to recruit participants in the classroom. Instead, participants were recruited via a recruitment post published in the September 2020 university student wellness online newsletter (see Appendix A), electronic advertisements posted by political science and biomedical science course instructors willing to assist with recruitment (see Appendix B), and flyers posted in an academic building (see Appendix B). Within the electronic advertisements a recruitment video was also included in addition to a link to the online 
consent form (see Appendix C). Electronic recruitment advertisements were sent from political science course instructors to over 100 students and from biomedical science course instructors to over 1,100 students via the Blackboard learning management system. The university student wellness online newsletter was sent via email to all 24,163 students enrolled at the university.

Students interested in participating reviewed and completed the online consent form prior to participation. The consent form was provided through Qualtrics, an online survey software, and provided to the students either via the written recruitment tools or by the researcher who emailed the students a link to this form (see Appendix D). After agreeing to participate in the study via the consent form, students began the researchercreated sociodemographic survey (see Appendix E) also within Qualtrics. Respondents reported their gender, age in years, race, body size with which they identified with, and academic college their declared major was within. Respondents also provided their university email address within the sociodemographic survey so the researcher could contact the students if they met the participant eligibility criterion. For students who met the eligibility criterion (i.e., self-identified body size of overweight or obese), the researcher emailed each prospective participant individually to schedule a virtual meeting. In these meetings, the researcher reviewed the completed informed consent form and provided photo-elicitation instructions (see Appendix F).

A total of 17 students completed the consent form and then the sociodemographic survey. After excluding those who self-identified as a person with normal weight or underweight body size, the number of participants included was eight; however only seven of the eight participants who met the study criterion participated in photo- 
elicitation $(n=7)$. The study received institutional review board approval by the institution before participant recruitment. Participants were assured confidentiality, that their participation was voluntary, and informed they could withdraw from the research at any time.

\section{Data Collection Tools}

Data was collected in five ways: (a) sociodemographic questionnaire, (b) body messages via photo-elicitation, (c) photo-elicitation questionnaire, (d) document collection of images of people from university's website, and (e) archival records of university documents.

Archival records included all university policies found in the study site's online policy library, the university's 2016-2021 long-range plan, and the university's diversity and inclusion implementation strategy. University policies provided data to answer research questions one and two. The university's 2016-2021 long-range plan, diversity and inclusion implementation strategy, in addition to body messages via photo-elicitation, the photo-elicitation questionnaire, and document collection of images of people from university's website all provided data to answer research questions three and four. Multiple data collection methods contributed to data triangulation and increased internal validity of the study (Creswell, 2014; Merriam \& Tisdell, 2016; Yin, 2014).

Sociodemographic questionnaire. Participants responded to a researchercreated questionnaire assessing their sociodemographics. Respondents reported their gender, age in years, race, body size with which they identified with, and academic college their declared major was within. 
Body messages via photo-elicitation. Photo-elicitation was requested of the participants. Merriam and Tisdell (2016) referred to this data collection technique as photovoice. Participants were asked to take five or more photographs over a two-week period, capturing examples of weight inclusive and weight stigmatizing university body messaging. This aligned with Bates, McCann, Kaye, and Taylor's (2017) researcher's guide for photo-elicitation. Data collection occurred asynchronously, from October 6, 2020 to November 15, 2020. Body messaging was defined as images of people and written content found on the study site campus or on the study site's social media platforms, and the messaging had to be distributed by the study site entity. For example, university banners highlighting students' accomplishments, university department marketing brochures, university social media sites, and university student organization materials met the messaging definition, while an advertisement for a job off-campus did not. Additionally, messaging in the dining centers did not qualify as those were managed by a contracted agency.

Photo-elicitation questionnaire. This researcher-created questionnaire (see Appendix G) was developed in Qualtrics, an online survey software. Participants stated what experiences, i.e., feelings or thoughts, the photo elicited in them, in addition to whether they found the body message to be weight inclusive or weight stigmatizing, why they chose to take the photo, and the location of the photographed item.

Participants used their own electronic device (e.g., smartphone, tablet) to take a photo and then they emailed the photo to the researcher who uploaded the photo to a secure online server. Participants included one of the following words, picture, photo, or images, in the subject line of the email. The researcher composed a rule in Microsoft 
Outlook for Outlook to send an automated message (see Appendix H) every time an email was received containing one of those words in the subject line. Thus, each time a photo was emailed to the researcher, the participant received an automated email providing the Qualtrics's survey link to the photo-elicitation questionnaire about the photographed item. This questionnaire asked participants to state what experiences, i.e., feelings or thoughts, the photo elicits in them, in addition to whether they found the body message to be weight inclusive or weight stigmatizing, why they chose to take the photo, and the location of the photographed item. The questionnaire took an estimated six minutes to complete. At the end of the study, all participant emails sent to the researcher containing the photos were deleted, and the photos are now only accessible through the secure online server.

Document collection online. Following traditional qualitative research methods, the researcher was an instrument for data collection (Merriam \& Tisdell, 2016). The researcher collected visual documents of images of people displayed on the university's website. Screenshots of images of people were captured on the study site's homepage, in addition to screenshots of images that were one-click away from the homepage. The exception were the university's Public Affairs Mission and Diversity webpages. All images within and one-click away from these webpages were collected. Furthermore, the content on the study sites homepage changed periodically due to the page including social media feeds. Due to this, images on the home page were collected daily for a twoweek period; from September 1, 2020 through September 15, 2020.

Archival records. The researcher collected archival records found on the university's website. Archival records included all university policies found in the study 
site's online policy library, the university's 2016-2021 long-range plan, and the university's diversity and inclusion implementation strategy. Records were collected if the documents included any of the following search terms: (a) weight, (b) obese or obesity, (c) overweight, (d) discriminate or discrimination, (e) inclusion or inclusive, (f) bias, (g) health or healthy, and (h) body mass index or BMI. An additional criterion for weight was records were only collected if weight was used as a noun in the context of body size versus as a verb referring to the value of an item.

\section{Data Analysis}

Qualitative content analysis. Archival documents and images of people collected from the university's webpages were analyzed via qualitative content analysis (Kuckartz, 2014). This type of analysis is also known as directed content analysis (Hsieh \& Shannon, 2005) and is recommended when the researcher desires to "validate or extend conceptually a theoretical framework or theory" (p. 1281). The content analysis followed Elo and Kyngäs's (2007) deductive approach. A priori codes were developed and integrated into the document policy analysis protocol (see Appendix I) and messaging analysis coding protocol (see Appendix J). These a priori codes were developed using language from: (a) the five principles of the HAES paradigm (ASDAH, 2018); (b) tenets of the weight-centered health paradigm outlined by O'Hara and Taylor (2018); (c) values and principles of the HAES paradigm outlined by O'Hara and Taylor (2018); (d) coding protocol of how people who are overweight or obese are portrayed in online news report developed by Heuer, McClure, and Puhl (2011); and (e) SELF magazine's style guidelines (Kylstra, 2018) for words and imagery related to weight (see Appendix K). Archival documents and images of people collected from the university 
webpages were reviewed and coded into the identified category the content corresponded with in the document policy analysis protocol or message analysis coding protocol.

Constant comparative analysis. Constant comparative analysis (Krueger \& Casey, 2015; Merriam \& Tisdell, 2016) was used to analyze the completed photoelicitation questionnaires. This analysis aligned with Creswell's (2016) eight steps for coding text:

(a) set up written content, transcripts, and questionnaires for easy coding; (b) read each text; (c) code each text; (d) list all codes; (e) group codes to remove redundancy and overlap; (f) compose a summary paragraph of the themes; $(\mathrm{g})$ create a concept map using the themes; and (h) develop a narrative linking together all themes. (p. 158)

Completed photo-elicitation questionnaires were coded using an inductive process, with in vivo coding being used, thus using the participants' words from the data to name codes (Creswell, 2016). To increase verifiability, the researcher conducted an audit trail to record this process. Merriam and Tisdell (2016) refer to an audit trail as a detailed account of how the study was conducted. The researcher kept a log of these processes, so another individual could follow the audit trail in the log to "authenticate the findings" (Merriam \& Tisdell, 2016, p. 252). In addition, this log aided the researcher when discussing findings of the research.

In the log the researcher included each collected photo, recording which participant took the photo or if the researcher took the photo, and the date each photo was captured. In addition, the photo-elicitation questionnaire completed by participants on each photo was included. All data was de-identified. Data was referred to as participant 
$(\mathrm{P})$, researcher $(\mathrm{R})$, photo $(\mathrm{PH})$, and questionnaire $(\mathrm{Q})$, and recorded as such. For example, in the audit trial log, participant one was P1 and the fifth photo they took was P1-PH5. When referring to the completed questionnaire for this photo, it was recorded as P1-PH5-Q5.

Reflexivity. To further increase the study's validity, the researcher engaged in epoche as described by Merriam and Tisdell (2016). Epoche is a process where the researcher increases awareness of and then sets aside their biases and judgements to get at the essence of what the participants are experiencing (Merriam \& Tisdell, 2016). The researcher is a registered dietitian nutritionist $(\mathrm{RDN})$ with training in nutrition and dietetics. While she self-identifies as a small person and was an outsider with her participants' body sizes, in her career as an RDN she developed deep empathy for people in larger bodies when she provided nutrition counseling to numerous clients who had deep resentment of their body size. She believes a person's health cannot be defined by their body mass index, although many people believe this to be truth as it is what modern-day society tells them and reinforces. The author is now an assistant professor at a public university and uses the HAES paradigm as her guiding perspective when instructing students. In this study, the researcher composed a researcher positionality statement where she discussed her experiences of weight stigma.

In addition, the researcher used bracketing of her thoughts and feelings as she conducted the data analysis. Merriam and Tisdell (2016) refer to bracketing as part of epoche in which the researcher temporarily sets aside biases so they can "examine consciousness itself" (p. 27). Through writing the researcher positionality statement and using bracketing, the researcher increased awareness of her biases about weight stigma. 
She then set those biases aside to do her best effort to analyze the data from an objective point of view.

\section{Limitations, Assumptions, and Design Controls}

Several limitations of this study should be noted. The first is participants selfidentified their body size as overweight or obese. The researcher chose not to use selfreported or researcher-measured height and weight to calculate a participant's body mass index (BMI) to place them in a BMI weight category. This was deliberate due to an individual's BMI not accurately reflecting how one self-identifies regarding body size. Furthermore, studies have found mixed findings regarding reliability of self-perceived weight status (Chang \& Christakis, 2003; Han et al., 2019; Pursey, Burrows, Stanwell, \& Collins, 2014). However, an accurate weight status was not essential to gain reliable data in this study. Instead, how the participant perceived themselves when compared to images viewed on campus or images on a university social media platform was an essential component of the study. It is assumed participants who self-identified as overweight or obese were more sensitive to weight stigmatizing and weight inclusive messaging, regardless of their BMI. Additionally, weight stigma also exists for people who are underweight (Puhl, Himmelstein, \& Quinn, 2018). With this study not including participants who self-identify as an underweight body size, the data is not generalizable to this population.

Another limitation included the small sample size, further decreasing the generalizability of the study. Due to COVID-19, the researcher was not permitted to recruit in person. Furthermore, students experienced more stressors due to COVID-19 in the recruitment period and it is assumed students did not have the time to commit to 
volunteer to be a participant. An additional limitation was the researcher found limited criteria to use to determine if written content and images of people were weight-inclusive, aligning with the HAES paradigm, versus weight-stigmatizing, aligning with the weightcentered health paradigm. Therefore, the researcher developed her own criteria for this, incorporating tenants from the HAES paradigm, the weight-centered health paradigms, coding criteria used in a content analysis study by Heuer, McClure, and Puhl (2011), in addition to style guidelines published by SELF magazine. These items were used by the researcher to develop the message analysis and document policy analysis protocols. These protocols were not tested for reliability and validity.

\section{Definition of Key Terms}

While weight stigma and weight bias are used interchangeably in the literature, the researcher has chosen to use the term weight stigma in her research. This decision was made after a review of the current published literature regarding obesity. In 2019, The Lancet had an initiative on nutrition and obesity, with a call for papers to help "eradicate weight stigma" (p. 168). Additionally, with the research on images used by the media of people who are overweight or obese, this literature primarily uses the terms weight stigma, obesity stigma, and stigmatizing images (Heuer, McClure, \& Puhl, 2011; Young, Subramanian, \& Hinnant, 2016).

Pescosolido and Martin (2015) provide definitions for the following terms used in this study.

Stigma. Stigma is defined as "a deeply discrediting attribute or mark of shame in which one's social identity is devalued" (p. 34). 
Stigmatization. Stigmatization is "a social process embedded in social relationships that devalues through conferring labels and stereotypes" (p. 34).

Stereotypes. Stereotypes are defined as "negative beliefs and attitudes assigned to labeled social entities" (p. 34).

Discrimination. Discrimination is referred to as "behaviors that act to endorse and reinforce stereotypes, and disadvantage those labeled" (p. 34).

In addition, according to Pescosolido and Martin (2015) stigma and stigmatization require four components: (a) distinguishing and labeling differences, (b) associating human differences with negative attributions or stereotypes, (c) separating "us" from "them," and (d) experiencing status loss and discrimination (p. 6).

Due to there not being a universal definition for weight stigma, the researcher proposes the following definition.

Weight stigma. Weight stigma is the devaluing of social identity due to an individual's body size.

People are stigmatized by others, whether this be anticipated stigma, perceived stigma, or received stigma (Pescosolido \& Martin, 2015). For example, anticipated weight stigma would be when an individual chooses not to receive medical treatment due to the expectation they will be devalued by a healthcare provider. Received weight stigma would be when an individual is denied medical treatment due to their body size, thus experiencing the devalue of their social identity.

Bias on the other hand refers to "an inclination of temperament or outlook" (Merriam-Webster, 2020) and is synonymous with prejudice. In the example above, the healthcare provider would be weight biased, while the client would experience weight 
stigma. Pescosolido and Martin (2015) used the term prejudice instead of bias. They define prejudice as the "endorsement of negative beliefs and attitudes in stereotypes" ( $p$. 34). This aligns with Puhl, Moss-Racusin, Schwartz, and Brownell's (2008) definition of weight bias, the "negative weight-related attitudes and beliefs that are manifested by stereotypes, rejection and prejudice toward individuals because they are overweight or obese" (p. 347). The exception is that these authors stated this is the definition for weight bias and weight stigma.

To further distinguish between weight stigma and weight bias, the researcher proposes the following definition for weight bias.

Weight bias. Weight bias is the negative weight-related attitudes and beliefs that are manifested by stereotypes toward individuals because of the individual's body size. This definition encompasses all body sizes, as weight bias can be displayed toward people of larger and smaller sizes.

Additionally, weight bias can lead to weight discrimination. Ramos-Salas, et al. (2017) define weight discrimination as the "unjust treatment of individuals because of their weight" (p. 1323).

\section{Significance of the Study}

This study contributes knowledge on whether students perceived body messages at a midwestern, master's comprehensive public university to be weight inclusive or weight stigmatizing, and the positive or negative effects of these messages. The researcher hopes this study will be used by the study site's stakeholders to consistently implement weight inclusive body messages, policies, and programs. This may contribute to increasing self-esteem and positive body image of students (Puhl \& Heuer, 2009). 
Findings from this present study may help guide higher education institutions in creating policies regarding inclusive images of people of different sizes. Additionally, increasing awareness of this modality of weight stigma for college students is needed. To increase this awareness, scholars have recommended strategies to include weight-neutral, HAES language (O'Reilly \& Sixsmith, 2012).

Additionally, to support health promotion policy efforts, health promotion campaigns may be implemented. Previous studies on health promotion campaigns showed many of these campaigns to increase weight bias, having unintended weight stigmatizing effects (Pearl, Dovidio, \& Puhl, 2015; Puhl, Luedicke, \& Peterson, 2013; Puhl, Peterson, \& Luedicke, 2013). It is essential health promotion campaigns use unbiased language such as that supported by HAES.

It is the researcher's hope this study will inform higher education institution constituents of how university body messaging can be used to promote weight inclusive environments. This will aid in decreasing weight stigma, possibly increasing recruitment, retention, and graduation for students who are overweight or obese. Lastly, the significance of the study includes increasing awareness of weight stigma as a social justice issue as described by Nutter et al. (2016), to promote a more inclusive learning environment. With limited literature existing on weight inclusive and weight stigmatizing body messages at higher education institutions, this study contributes knowledge and awareness of how university messaging affects the college student experience. 


\section{Summary}

Weight stigma is pervasive in North American society (Puhl \& Heuer, 2010; Ramos-Salas et al., 2017), yet there is limited research on how students who are overweight or obese experience weight stigma at higher education institutions through institutional messages. In addition, higher education institutions have policies related to diversity and inclusiveness, yet within these policies, weight discrimination is rarely addressed. This study contributes knowledge on what weight inclusive and weight stigmatizing body messages college students experience at a public university. This study also examined university policy, initiatives, and images of people used on the university's website for weight inclusive and weight stigmatizing body messages.

By using the HAES paradigm (ASDAH, 2018) as the guiding theoretical framework, this study was driven through a social justice lens. University body messages were analyzed for weight inclusive and weight stigmatizing content, and college students' perspectives of how text and images of people in these messages affected them were gained. Additionally, university policies, initiatives, and images of people used on the university website were analyzed for weight inclusive and weight stigmatizing content. Data from this study may lead to consistent implementation of weight inclusive body messages at the university, adoption of weight inclusive university policies and programs, and increased awareness of weight stigma as a social justice issue. This may aid in decreasing weight stigma, possibly increasing recruitment, retention, and graduation for students who are overweight or obese. 
SECTION TWO:

PRACTITIONER SETTING FOR THE STUDY 


\section{Introduction}

This section begins with a brief history of the study site, including discussion of the site's public affairs mission and values. An organizational analysis is then provided where the process of how policies are approved is described. The section concludes with a leadership analysis followed by an examination of implications for research in the practitioner setting, and a concluding summary.

\section{History of Organization}

In 1905, the study site was founded as the Fourth District Normal School, where the organization served to guide students in teacher preparation. This university has continued to evolve since then, now providing undergraduate, graduate, and doctoral programs. In 2020, over 24,000 students were registered within its four campuses (MSU Office of Institutional Research, 2020a).

\section{Public Affairs Mission}

In 1995, the state legislature approved designating the site with a mission in public affairs (MSU, 2018a). Within this mission are tenants referred to as the three pillars of public affairs: (a) ethical leadership, (b) community engagement, and (c) cultural competence (MSU, 2018a). The public affairs pillars are incorporated into the vision of the study site to "be the university of choice to develop successful students who excel academically and in ethical leadership, cultural competence, and community engagement" (MSU, 2018b, Vision section, para. 2). The three pillars of public affairs are described below.

This midwestern, master's comprehensive university strives to develop students to be ethical citizen scholars who demonstrate leadership (ethical leadership), in addition to 
increasing student knowledge and respect for individuals of varying backgrounds (cultural competence) and recognizing the value of sharing knowledge to their communities (community engagement). The community engagement pillar also emphasizes students recognize the value of "scientific principles in the generation of sound public policy" (MSU, 2018b, Community Engagement section, para. 8). These pillars are the driving force behind many campus events including the annual public affairs and collaborative diversity conferences, and the hunger banquet. In addition, the pillars are woven into the university's academic curriculum and assessed by items on the university undergraduate exit survey.

\section{Values}

Values of the study site include inclusiveness, promoting an environment of respect for all individuals, and continuous improvement in programs and policies (MSU, 2018b). This university categorizes inclusiveness with being fair, equitable, and valuing social justice. These items are defined as "the recognition that each person possesses not just one, but multiple identities; and the celebration of the similarities, as well as the differences, of our civility, trust and collaboration" (MSU, 2018b, Values section, para. 3).

The values of diversity and inclusion are represented together as a key area in the study site's 2016-2021 Long-Range Plan. Diversity and inclusion university goals in the long-range plan include the following (a) "support initiatives to encourage discussion of, and appreciation for, differences"; and (b) "implement effective training and/or professional development to increase cultural consciousness/competence in diversity and 
inclusiveness for students, faculty, and staff" (MSU, 2016a, University goals section). Strategies to address the diversity and inclusion goals include:

(a) "embrace diversity in all its forms to be true to the university's values and achieve the university's goals; (b) embrace a system-wide approach to create an environment where all groups and individuals can thrive and achieve academic and personal success; and (c) lead by example in promoting inclusiveness within the community, region, and state" (MSU, 2016a, Strategic priorities section).

\section{Organizational Analysis}

The midwestern, master's comprehensive public university is an organization with over 3,700 employees (MSU, 2019a). To support this size of a bureaucracy, the organizational structure has several components. These components align with Mintzberg's (1979/2005) five basic parts of organizational structure. At the top is the strategic apex. Employees found in this component Mintzberg (1979/2005) refers to as "top-level managers" (p. 223). Their responsibility is to carry out the organization mission in addition to serving "the needs to those people who control or otherwise have power over the organization" (Mintzberg, 1979/2005, p. 223). The Board of Governors are at the highest level of the study site's strategic apex, followed by the president and director of internal audit and risk management. Positions serving the needs of the president and who also report to the president and are included in the strategic apex are the general counsel, the director of institutional equity and compliance, the provost, the director of athletics, the assistant to the president and chief diversity officer, and the vice president for student affairs. 
Mintzberg (1979/2005) refers to employees in the middle line as "middle-line managers" (p. 224). At the study site, middle line managers have numerous titles. These include associate provosts, vice presidents, or directors and these positions report to toplevel managers in the strategic apex. For example, the deputy provost, associate provost of access and outreach, and associate provost of student development and public affairs all report to the provost. Additionally, looking specifically at the associate provost of student development and public affairs there are several directors who report to that position who are also part of the middle line. This includes the director of student learning services, director of learning commons, and director of community-engaged learning.

Furthermore, college deans and department heads compose the middle line in Mintzberg's (1979/2005) organizational structure. Department heads report to the deans in the strategic apex, while the deans' direct line of authority is the provost also in the strategic apex. In addition, positions reporting to the vice president for student affairs in the strategic apex include the assistant vice-president dean of students, associate vicepresident of enrollment management, director of residence life, and director of the student union.

The bottom of Mintzberg's (1979/2005) model is the operating core. The operating core is who does "the basic work related directly to the production of products and services" (Mintzberg, 1979/2005, p. 223). This is composed of faculty who strive to meet the study site's mission by producing students to be global citizen scholars. The line of authority for faculty are department heads. 
The strategic apex, middle line, and operating core need additional help to support the operations at the study site. Mintzberg (1979/2005) refers to these entities as the technostructure and support staff. The technostructure is one step removed from the organization's workflow. Responsibilities in these positions do not include teaching, but instead assist faculty and students in the teaching and learning process. At the study site, this includes computer technicians, budget analysts, staff at the faculty teaching and learning center, and the center for learning and writing. The responsibility of support staff is to facilitate needs of the organization (Mintzberg, 1979/2005). Administrative assistants, groundskeepers, custodians, and dining center staff are examples of support staff at the study site. All five parts of the organizational structure cannot exist independently and require one another to meet the mission and goals of the university.

\section{Policy Process}

The midwestern, master's comprehensive public university as an organization aligns with the bureaucratic model (Manning, 2013). This university has a hierarchical structure whereas those with the most power are at the top and decision making is a top down dissemination (Manning, 2013). In addition, there are operating procedures published as organizational policies. These policies are located online and referred to as the policy library (MSU, 2019b).

Policies are grouped into either the governing category or the operating category. Governing policies includes procedures regarding Board of Governors' functioning and governance of all constituents including students. While governing policies refer to policies established by the Board of Governors, operating policies are established by the 
university president. An operating policy “interprets and implements Governing Policies" (MSU, 2019b, Policy Library section, para. 2).

Governing and operating policies are indexed within 12 categories including administrative, academic, student, human resource, and health and wellness policies. Governing policies include but are not limited to governing policies for faculty, staff, and students. The overarching governing policy for faculty is G3.03, the faculty handbook. This governing document outlines many of the operating policies for the university's faculty. For staff, the overarching governing policy is G7.02, the employee handbook for administrative, professional, and support staff employees. Like the faculty handbook, the employee handbook outlines many operating policies, but for the study site's staff and not faculty. Lastly governing policy, G5.01, the code of student rights and responsibilities, outlines many of the operating policies for students. Additional governing policies include the facility rental policy, information assurance policy, and university fleet vehicle policy.

The process of adopting or revising a policy at the study site follows the bureaucratic communication pattern discussed by Manning (2013). Line of authority is followed, requests must be written requests, and all procedures are to be documented through a "paper trail" (Manning, 2013, p. 118). According to the study site's Policy Library website (2019), policy adoption or revision procedure begins with the proponent of a new policy or policy revision sending the policy to the appropriate vice presidentlevel administrator for consideration. This administrator then forwards the proposal to the president or administrative council (AC) for review. AC at the study site consists of 17 members including the president, the provost, one academic college dean, one 
member of general counsel, the chief diversity officer, and the vice president for student affairs. If and when the proposal is approved by the $\mathrm{AC}$ or president, the policy is forwarded to the office of general counsel for inclusion into the policy library (MSU, 2019b).

Although the procedure above is the current published process for policy adoption and revision, the study site now has an additional method for policy review (S. Res. 7, 2017). In 2017, an ad hoc Faculty Senate committee was formed to investigate the policy library. This committee was formed to determine the role faculty had in policy development and consideration of new policies in this policy library, in addition to what role faculty should have in the process.

It was concluded that faculty involvement is "inconsistent and potentially inadequate" (MSU Faculty Senate Agenda, 2017, p. 55). The recommendation by this ad hoc committee was to form a standing committee on policy review. Membership on the committee was to include faculty and a student representative to increase faculty and student participation in policy adoption and revision. This recommendation aligns with the American Association of University Professors' (AAUP) Statement on Government of Colleges and Universities (AAUP, 1966). In this statement, shared governance is advocated for, where administrators, faculty, staff, and students all have "appropriately shared responsibility and cooperative action among the components of the academic institution" (AAUP, 1966, Statement on Government of Colleges and Universities, para. $1)$.

The Committee on Policy Review was established at the start of the 2017-2018 academic year (S. Res. 7, 2017). Membership consisted of three faculty members, one of 
whom was committee chair, in addition to the chair of faculty senate, chair-elect of faculty senate, and chair of the faculty handbook revision committee. The director of human resources, a representative from the registrar's office, and a representative from the Student Government Association were also included as committee members, although these were ex officio members, not having voting privileges. Lastly, members from the strategic apex included the provost and the chief general counsel who were also ex officio members.

This committee has several purposes with one being to meet twice a month to “discuss pending policies under consideration by university leadership" (S. Res. 7, 2017, Purpose section, para. 1). Another purpose is to develop and deliver reports to Faculty Senate regarding pending operating policies being considered by university leadership. The Committee on Policy Review provides an additional method for faculty to have new policies reviewed and considered for adoption. Furthermore, existing policies warranting review and pending policy changes to an existing policy can be brought by faculty to this committee for review.

In policy analysis, Bardach and Patashnik (2016) recommended analysts follow the Eightfold Path method. In the first seven steps of this method, an individual (a) defines what the problem is, (b) assembles evidence to justify the problem and assess the policy context, (c) constructs alternative policy options to address the problem, (d) selects criteria to project outcomes of the policy options, (e) projects outcomes based on chosen criteria, (f) confronts trade-offs of each policy option, and (g) selects the policy option or options to propose to key constituents (Bardach \& Patashnik, 2016). At the study site, faculty have the right to complete these seven steps. The faculty member who becomes 
the policy advocate would then complete the eighth and final step of Bardach and Patashnik's (2016) Eightfold Path, telling the story, to the Committee on Policy Review members.

The faculty policy advocate would need to prepare an executive summary and policy brief of the proposed policy options to send to the Committee Chair. The policy options may include proposing a new policy, a revision to an existing policy, or consideration of a pending policy change to an existing policy. In these documents it is recommended to not use quantitative data alone, but to also "put a human face on the problem" (Bardach \& Patashnik, 2016, p. 74). Additionally, policymaking is not a "oneshot affair" (Bardach \& Patashnik, 2016, p. 41), and thus the policy advocate needs to invest time gaining support from key constituents for the proposed options. These constituents at the study site would include members of the Committee on Policy Review.

Once a policy is adopted, it is indexed in the policy library by Web and New Media Services. Each indexed policy identifies the line of authority. This includes the administrator and office responsible for the policy and the contact person within that office. For example, in the prohibition of discrimination and harassment policy, operating policy $1.02-8$, the line of authority is the vice president for diversity and inclusion found within the office for institutional equity and compliance. The director of institutional equity and compliance is the contact person within the office.

The study site is a bureaucratic institution whose structure aligns with Mintzberg's (1979/2005) five basic parts of an organization. The president and Board of Governors in the strategic apex have the most power, followed by department heads and deans in the middle line, and then down to faculty in the operating core. In the past, the 
power to adopt or revise policies primarily was given to administrators in the strategic apex and middle line. Recently though, the study site created the Committee on Policy Review. Membership on this committee includes faculty and a student representative, therefore increasing shared governance on policymaking as advocated by the AAUP. Faculty can provide this committee with policy analysis documents of new policies for consideration, revisions to an existing policy, or a pending policy change to an existing policy.

\section{Leadership Analysis}

The study site's vision is to "be the university of choice to develop successful students who excel academically and in ethical leadership, cultural competence, and community engagement" (MSU, 2018b, Vision section, para. 2). To be the university of choice, university leaders have implemented adaptive leadership principles. According to Northouse (2016), adaptive leadership refers to how leaders prepare and motivate their followers to adapt to change within the greater social system. Higher education institutions have experienced changes in the demographics of the students they serve in addition to increased external pressures on accountability for student outcomes. To align with the study site's vision, their leaders have used adaptive leadership to adapt to these changes.

Heifetz (1994) and his colleagues developed the adaptive leadership framework published in the book, Leadership Without Easy Answers. Heifetz identified several characteristics of this leadership approach which help explain the spirit of it. One of these characteristics is mobilizing, motivating, organizing, orienting, and focusing the attention of others. Through this process Heifetz stated adaptive leaders themselves may 
have a progression in their values, in addition to engaging others in "adjusting their values, changing perspectives, and developing new habits of behavior" (p. 276). Adaptive leadership involves followers at all levels, from the strategic apex to the operating core, encouraging each member of the organization to provide input to solve complex problems.

Additionally, adaptive leadership characteristics include incorporating four different perspectives. There are the systems, biological, services, and psychotherapy perspectives (Northouse, 2016). Taking a systems perspective acknowledges problems are complex, dynamic, and part of an interactive environment. The biological perspective acknowledges people are equipped with the ability to adapt to internal and external environments and can thrive in changing environments. Incorporating a service orientation refers to how a leader uses their authority. An adaptive leader uses their status "to serve" (Northouse, 2016, p. 260) followers; focusing on how leaders can support followers to adapt to changing environments. Lastly, the psychotherapy perspective is the perspective that people need to be in supportive environments to adapt to change.

State appropriations to fund higher education have been decreasing since the 1990’s (Loss \& McGuinn, 2016), increasing the necessity for a steady to increased student enrollment to fund universities. Fall 2019 total student enrollment at the study site declined $1.03 \%$ from fall 2018 (MSU, 2019c). University leaders embraced the systems perspective of adaptive leadership (Northouse, 2016) to address this problem. They assembled, motivated, and focused the attention of faculty and staff by forming strategic enrollment (SEM) councils. SEM councils were comprised of university faculty 
and staff and were charged with developing goals and action plans regarding recruitment, retention, delivery of academic programs, and marketing (MSU Board of Governors, 2019).

Furthermore, the study site had a $13.2 \%$ decline in nonresident alien or International students from 2014 to 2018 (MSU Office of Institutional Research, 2020b). The study site's leaders' actions in how to respond to this situation aligned with adaptive leadership's systems, biological, services, and psychotherapy perspectives. Instead of solely determining this decrease was due to external policy, study site leaders chose to have an international committee comprised of faculty and staff explore, develop, and implement strategies to increase enrollment for this student population (Clif's Notes, 2019, October 15). International enrollment increased significantly in fall of 2019, almost eliminating the $13.2 \%$ loss.

Regarding diversity in the student population, the study site's student enrollment from 2014 to 2018 had a $10.5 \%$ increase in Hispanic/Latino students, $22.1 \%$ increase in Asian students, $10.7 \%$ increase in African American students, and a 38.3\% increase in students who self-report as two or more races (MSU Office of Institutional Research, 2020). Additionally, 34\% of the study site's undergraduates are first-generation college students (MSU, 2019d). With a change in student demographics, this university adapted by implementing additional student initiatives and student support services. These changes align with adaptive leadership's systems, biological, services, and psychotherapy perspectives. New programming included initiatives through multicultural services located within the Division of Student Affairs and first-generation student support found in the Center for Academic Success and Transition. 
Additionally, to aid in fostering inclusive learning environments for students of underrepresented groups, a program called Shattering the Silences was initiated in 2013 (MSU, 2019e). These sessions were designed to promote dialogue versus debate regarding diversity. Students, faculty, and staff were all invited to join. Past sessions discussed topics about diversity including LGBTQ+, African diaspora, Black Lives Matter, and people with disabilities. There have not been any sessions addressing weight stigma.

State appropriations are partly determined for each institution by outcomes on the institution's performance indicators (Loss \& McGuinn, 2016). Indicators include student retention, and graduation and job placement rates. The student initiatives and student support services discussed above are provided to aid in student success and thus student retention and graduation rates. For this study site to be the university of choice, its leaders have used adaptive leadership principles to adapt to changing environments. Adapting to change will aid the university in achieving this vision, in addition to meeting their performance indicators and thus increasing state appropriations.

\section{Implications for Research in the Practitioner Setting}

While weight stigma is pervasive in North American society (Puhl \& Heuer, 2010; Ramos-Salas et al., 2017), little research exists on the sources (e.g., messaging) contributing to weight stigma in higher education. Furthermore, there is scarce research on analyzing higher education policies that promote weight inclusive environments. With the American College Health Association (2020) reporting 37\% of college students are overweight or obese, the implications for weight inclusive body messaging and policy analysis research are numerous. 
The study site values include inclusiveness and promoting an environment of respect for all individuals. This research could benefit the university by increasing awareness of and providing knowledge on whether or not body messaging at this university promotes weight inclusivity; do students in larger bodies experience weight stigma from university messaging? In addition, this research will provide knowledge regarding whether policies or images of people used on the university's website promote weight inclusiveness. The university has student initiatives, student support services, and professional development opportunities to aid in building inclusive learning environments for but not limited to people of color, people who identify as non-binary or ungendered, and people with disabilities. If policies and services regarding weight inclusiveness do not exist, this research could provide recommendations to the study site's administrators on how to include these.

Research results and recommendations in the form of an executive summary and policy brief could be provided to university constituents, including the Committee on Policy Review. The researcher could request being a guest at one of this committee's monthly meetings to share the implications and recommendations from this study. This would provide the researcher with a seat at the table, where she could have influence due to being able to be an active participant at the meeting as discussed by Cervero and Wilson (2006). With the provost and chief general counsel being members of the Committee on Policy Review, this could also put her recommendations into the hands of administrators in the university's strategic apex.

Although there is limited research on weight stigma experienced by college students, the existing research does indicate college students experience this stigma (Puhl 
\& Brownell, 2006; Trainer, Brewis, Williams, \& Rosales-Chavez, 2015). Research has suggested the effects from weight stigmatizing experiences are detrimental to academic self-efficacy (Aimé et al., 2017) and self-esteem (Stevens, 2018) of students.

Additionally, research has found young adults who are obese are less likely to attain a college degree (Fowler-Brown et al., 2010), especially females (Benson, von Hippel, \& Lynch, 2017; Crosnoe, 2007). Therefore, an additional benefit to the study site could be using the findings from this study to adapt images of people and written text on campus to be weight inclusive which may have a positive effect on students' academic selfefficacy and student recruitment.

\section{Summary}

The study site is a midwestern, master's comprehensive university with a mission in public affairs. This bureaucratic institution values inclusiveness, promoting an environment of respect for all individuals, and continuous improvement in programs and policies (MSU, 2018b). To aid in improving policy and aligning with adaptive leadership principles, the site created the Committee on Policy Review. This committee provides a method for faculty to have new policies reviewed and considered for adoption, in addition to challenging existing policies to be considered for review.

Thirty-seven percent of college students are overweight or obese (The American College Health Association, 2020) and weight stigma continues to increase (Andreyeva, Puhl, \& Brownell, 2008). Young adults who are obese are less likely to attain a college degree (Fowler-Brown et al., 2010), especially females (Benson, von Hippel, \& Lynch, 2017; Crosnoe, 2007), and research suggests the effects from weight stigmatizing experiences are detrimental to academic self-efficacy (Aimé et al., 2017) and self-esteem 
(Stevens, 2018). Furthermore, stigmatizing beliefs and attitudes are maintained and reinforced with exposure to negative messages toward people of larger size. This research could benefit the study site by increasing awareness of and providing knowledge on whether or not body messaging at the site promotes weight inclusivity. In addition, this research could provide knowledge regarding if policies exist to promote weight inclusiveness. 
SECTION THREE:

SCHOLARLY REVIEW FOR THE STUDY 


\section{Introduction}

Weight stigma has been described as one of the last socially acceptable forms of discrimination (Puhl \& Brownell, 2001; Vartanian, Pinkus, \& Smyth, 2014), with the prevalence of self-reported perceived weight discrimination increasing from $7.3 \%$ in 1995-1996 to $12.2 \%$ in 2004-2006 (Andreyeva, Puhl, \& Brownell, 2008). Many higher education institutions have policies related to diversity and inclusiveness, yet within these policies, weight discrimination is rarely addressed. In addition, there is limited research on how university body messaging promotes or impedes a weight inclusive environment.

The researcher, therefore, investigated how weight inclusive and weight stigmatizing body messages were included in university policy and through images of people used on the university's website, if at all. In addition, the researcher explored college student perspectives of body messaging at a public university, and whether the messages used weight inclusive text and images of people, or weight stigmatizing text and images of people. It is the researcher's hope this study will increase awareness of weight stigma and contribute knowledge toward how the messaging of higher education institutions can be used to promote weight inclusive environments. Promoting weight inclusive environments would then aid in decreasing weight stigma for students who are overweight or obese.

This scholarly review begins with a review of the literature on a weight inclusive theoretical model. A synthesis and critique of the literature on weight stigma within higher education, and weight stigma and imaging are then provided. This is followed by a summary of the literature on weight discrimination and policy. The paper concludes with a summary of the review. 


\section{Health at Every Size ${ }^{\circledR}$ Theoretical Model}

The theoretical model for this study is the Health at Every Size (HAES $\left.{ }^{\circledR}\right)$ paradigm. (Health at Every Size and HAES are registered trademarks of the Association for Size Diversity and Health and used with permission.) The HAES model is a weight inclusive versus weight normative approach to policy and individual decision making, grounded in a social justice framework (Association for Size Diversity and Health [ASDAH], 2018). The weight inclusive approach emphasizes "viewing health and wellbeing as multifaceted while directing efforts toward improving health access and reducing weight stigma" (Tylka et al., 2015, p. 1). In contrast, the weight normative approach emphasizes "weight and weight loss when defining health and well-being" (Tylka et al., 2015, p. 1).

In 1997, Jon Robison published an article on a paradigm for health educators to use when educating about weight management. He referred to this paradigm as the "alternative paradigm" (p. 29) for weight management. It included the principle of selfacceptance whereas educators where to "provide affirmation and reinforcement of human worth irrespective of differences in physical size and shape" (p. 31).

At the beginning of the 21st century this alternative paradigm was referred to as the Health at Any Size paradigm (Berg, 1999; Miller \& Jacob, 2001). In 2003, the Association for Size Diversity and Health (ASDAH) was formed, and the creators of this organization changed the paradigm's name from Health at Any Size to Health at Every Size (ASDAH, 2018). In addition, ASDAH established the original HAES principles (ASDAH, 2018). Focusing on how to improve healthy living instead of focusing on weight is the HAES model's foremost foundation (ASDAH, 2018). ASDAH revised 
these principles in 2013 and these remain as the guiding principles (ASDAH, 2018). The HAES principles are:

1. Weight inclusivity: Accept and respect the inherent diversity of body shapes and sizes and reject the idealizing or pathologizing of specific weights.

2. Health enhancement: Support health policies that improve and equalize access to information and services, and personal practices that improve human wellbeing, including attention to individual physical, economic, social, spiritual, emotional, and other needs.

3. Respectful care: Acknowledge our biases, and work to end weight discrimination, weight stigma, and weight bias. Provide information and services from an understanding that socioeconomic status, race, gender, sexual orientation, age, and other identities impact weight stigma, and support environments that address these inequities.

4. Eating for well-being: Promote flexible, individualized eating based on hunger, satiety, nutritional needs, and pleasure, rather than any externally regulated eating plan focused on weight control.

5. Life-enhancing movement: Support physical activities that allow people of all sizes, abilities, and interests to engage in enjoyable movement, to the degree that they choose. (ASDAH, 2018)

In contrast, the weight normative approach aligns with the weight-centered health paradigm (O’Hara \& Gregg, 2006; O’Reilly \& Sixsmith, 2012), also known as the weight-focused paradigm (Bacon \& Aphramor, 2011). Principles of this paradigm emphasize that an individual's health status can be estimated by their body mass index 
(BMI), excess weight increases morbidity and mortality risk, and an outcome of losing weight is improved health (O'Hara \& Gregg, 2006). These principles have been refuted in the literature.

In Bacon and Aphramor's (2011) review of the weight-centered health paradigm's principles compared to the HAES paradigm's principles, they found most studies on BMI and mortality to show that people who are overweight (BMI range of $25-29.9 \mathrm{~kg} / \mathrm{m}^{2}$ ) or moderately obese (BMI range of $30-34.9 \mathrm{~kg} / \mathrm{m}^{2}$ ) have the same or longer life spans than people in the normal weight BMI category $\left(18.6-24.9 \mathrm{~kg} / \mathrm{m}^{2}\right)$. Janssen and Mark (2007) found comparable results in their review. They conducted a meta-analysis, combining data from 32 articles to examine the relationship between BMI and mortality in individuals 65 years of age or older. These articles provided 28 subgroups that the researchers categorized by BMI specification.

In 13 of the 28 subgroups, mortality risk was lower for participants in the overweight BMI category than in the normal weight BMI category, while in 11 of the 28 subgroups this was reversed (Janssen \& Mark, 2007). In those 11 subgroups, mortality risk was lower for participants in the normal BMI category than those in the overweight category. Furthermore, in four of the subgroups lowest mortality risk presented for participants in the obese BMI range. Janssen and Mark's meta-analysis suggests that the overweight BMI range is not associated with a decreased life span in older adults. In addition, the obese BMI range was associated with only a slight increase in mortality risk of about $10 \%$ in older adults.

Contradicting the weight-centered paradigm principle that losing weight results in better health, data suggests weight loss can increase mortality risk. Ingram and 
Mussolino (2010) conducted a longitudinal study using National Health and Nutrition Examination Survey (NHANES) data. The sample was 6,117 multi-ethnic adults, aged 50 years or older. The relationship between weight loss, BMI, and mortality was investigated. Baseline data was collected from 1988 to 1994, and mortality follow-up was conducted through 2000. Results showed weight loss of $15 \%$ or more of baseline body weight to be associated with an increased risk of mortality from all causes for adults who were overweight.

Regarding the HAES paradigm, Bacon and Aphramor (2011) reviewed six randomized controlled trials (RCTs) comparing this paradigm to the weight-centered health paradigm, for size acceptance changes of the participants. In the four studies that measured participants' psychosocial outcomes, 100\% of the studies reported positive statistically significant results regarding mood, self-esteem, and body image for those in the HAES groups. In only one of those four studies did the participants in the weightcentered group have improvement in psychosocial outcomes. In addition, three studies measuring physiological indices resulted in improvements in blood pressure, serum cholesterol levels, and health behaviors such as physical activity for participants in the HAES groups. While in the weight-centered groups, in only one study did participants have improvements in the physiological outcomes.

Tylka et al. (2014) conducted a review on weight-inclusive versus weightnormative approaches in health care and public health settings. Results showed weightnormative approaches to increase healthcare professionals' weight bias in addition to increasing the internalization of weight stigma by their clients. For example, when healthcare professionals blame the individual when weight loss is not achieved, this 
increases weight stigma for the client. When this stigma is internalized and clients blame themselves for not being able to lose weight, clients are at a higher risk of developing negative psychological effects such as depression (Tylka et al., 2014). When this stigma is perceived as weight discrimination, individuals who experience it are 2.41 times more likely to develop more than three psychiatric diagnoses (e.g., mood disorder, anxiety disorder, substance disorder) than individuals who do not perceive weight discrimination (Hatzenbuehler, Keyes, \& Hasin, 2009).

Mensinger, Tylka, and Calamari (2018) found comparable results. A crosssectional survey was distributed and included questions about stigmatizing situations, weight bias internalization, body-related shame and guilt, and healthcare stress. The survey was completed by 313 female participants of varying body sizes with BMIs categorizing participants in all weight categories from underweight to obese. Results showed that participants with higher BMIs experienced greater amounts of weight stigma which then created a cascade of events. Higher weight stigma contributed to greater feelings of body-related guilt and body-related shame, which was associated with greater healthcare-related stress and then greater avoidance of healthcare. The authors recommended a weight inclusive approach for the healthcare setting.

While all five of the HAES principles encompassed the spirit of this study, the weight inclusivity and respectful care principles of the HAES model were used to help guide the research methods. These principles were chosen due to aligning with the study's research questions. Documenting and analyzing university body messages and policy for weight inclusive and weight stigma discourse provided data on whether or not university messages and policies portrayed weight inclusivity. In addition, this study 
provided data which aided in illuminating whether or not the university had actions contributing to weight stigmatizing environments that perpetuated this stigma and weight discrimination. Having knowledge on how students who are overweight or obese experience weight stigma within higher education could provide implications for what the university can do better to provide weight inclusive environments.

\section{Weight Stigma}

Extending from Pescosolido and Martin's (2015) definition of stigma, the researcher proposes the definition of weight stigma as the devaluing of social identity due to an individual's body size. The consequences of weight stigma are severe, including having a negative effect on the psychological and physical health of individuals who experience it (Gloor \& Puhl, 2016; Puhl \& Heuer, 2009). Examples of these negative effects are depression, lowered self-esteem, body image dissatisfaction, binge-eating behaviors, and reduced or avoidance of physical activity (Puhl \& Heuer, 2009). In the following section, the literature on weight stigma and higher education is reviewed, followed by a review of the literature on weight stigma and images.

\section{Higher Education}

The American College Health Association (2020) reported 37\% of college students have overweight or obesity. Research has found young adults who are obese are less likely to attain a college degree (Fowler-Brown et al., 2010), and it is speculated weight stigma contributes to this. While weight stigma is pervasive in North American society (Puhl \& Heuer, 2010; Ramos-Salas et al., 2017), little research exists on the extent, source, and modality that contributes to weight stigma on college campuses. Vartanian, Pinkus, and Smyth (2014) conducted a phenomenological study in Sydney, 
Australia about weight stigma. Their participants were not college students; the mean age of the 46 participants was 28.4 years $(\mathrm{SD}=21.16)$ and $64.4 \%$ were single. Participants experienced an average of 11.12 episodes of weight stigma over the twoweek period, with a range of 1 to 49 episodes over the time period. The most frequently reported sources of weight stigma were strangers (18.4\%), partners (16.2\%), friends $(15.8 \%)$, parents $(10.8 \%)$, and media $(10.6 \%)$. These sources of weight stigma compare with Puhl et al.’s (2008) results of weight stigma prevalence sources being friends $(15.8 \%)$, parents $(12.7 \%)$, strangers $(9.8 \%)$, and partners $(9.5 \%)$. In addition, the most common location of weight stigma episodes was at home (Puhl, Moss-Racusin, Schwartz, \& Brownell, 2008; Vartanian et al., 2014). The most frequently (76.6\%) reported expression of weight stigma was verbal (Puhl et al., 2008).

Stevens (2018) interviewed 14 students at two midwestern state universities on their experiences of weight stigma and discrimination in the university setting. Although Stevens (2018) reported her participants had experienced weight stigma in dormitories and family homes, she found the primary locations of experiencing this stigma were in classrooms, dining centers, the campus recreation center, and social drinking places. To manage this stigma, participants attempted to avoid these spaces. Stevens (2018) stated, "fat students are bombarded with antiobesity information around campus" (p. 136). The consistent rhetoric to lose weight contributes to individuals having lower self-esteem and poor body image (Stevens, 2018; Wellman et al., 2018). Although Stevens's study did not include document collection, her participants noted that documents on campus (e.g., campus newspaper, signs in bathroom stalls, materials at student health center) triggered feelings of weight stigma. 
Terminology. Trainer et al., (2015) conducted research on college students' terminology when describing body shapes and sizes. A random sample of 202 students were asked to provide adjectives to describe different shapes of male and female silhouettes. Twenty-one adjectives were identified and integrated into a survey. The survey was taken by 264 participants, purposefully selected at different geographic regions on a college campus. This survey evaluated to what extent students were likely to use each adjective to describe someone else's weight, and which adjectives they would prefer someone to use when speaking with them about their weight. Additionally, 15 students were interviewed to further explore size terminology usage.

Survey results indicated the terms "fat" and "obese" were not preferred language, and "overweight" and "heavy" were preferred (Trainer et al., 2015). Nevertheless, in interviews, the word fat was reported to be used extensively. Trainer et al.'s (2015) recommended interventions included providing anti-weight bias programming for college students, peer-support groups on body image and health promotion in residence halls, and strategically posting informational posters around the college campus about how to decrease weight stigma. Anti-weight bias and weight-inclusive approaches have shown to be effective in reducing weight bias and weight stigma on college campuses (Humphrey, Clifford, \& Neyman-Morris, 2015; Rote, Love, Lakatos, \& Hewitt, 2018; Webb, Fiery, \& Jafari, 2016).

Gender. Trainer et al.'s, (2015) results also indicated college females who were overweight or obese were more likely to be described negatively and experience weight stigma than males. Experiencing weight stigma by one's family has also shown to occur more for females than males $(N=4,990 ; 50 \%$ female; Sutin \& Terracciano, 2017). 
Furthermore, in Crosnoe's (2007) study using data from the National Longitudinal Study of Adolescent Health ( $n=4,865$ females; $n=4,055$ males), he found females who were obese to be $50 \%$ less likely to attend college than females who were not obese.

Differing from these studies, Puhl and Brownell (2006) found that males who were overweight or obese were more likely to experience weight stigma within education than females who were overweight or obese. Participants $(N=222 ; 50 \%$ male, 50\% female) were recruited from members in a national weight loss support group organization. Twenty-seven percent of males had experienced weight stigma from a teacher or professor, and $18 \%$ of females had experienced this source of stigma. Using the same recruitment tool and having only female participants, Puhl and Brownell repeated the study and discovered that $32 \%$ of adult females $(N=2,449)$ had experienced weight stigma from a teacher or professor, with $21 \%$ experiencing this stigma from a teacher or professor more than once.

Grade point average. Weight stigma from instructors may contribute to decreased academic self-efficacy in college students (Aimé, Villatte, Cyr, \& Marcotte, 2017). A study about BMI and grade point average (GPA) in higher education suggested these variables are inversely related (Aimé et al., 2017) although there are many confounding variables such as socioeconomic status, financial support, and parental support. Aimé et al.'s, (2017) research on BMI and GPA controlled for father's education level and financial support from parents. Coefficients used were academic self-efficacy and class attendance. Participants were 298 college females located at a university in Quebec, Canada. 
BMI was determined to be a significant predictor of academic self-efficacy $(\beta=$ $-.089 ; t=-2.306 ; p=.021)$ and class attendance $(\beta=-.027 ; t=-2.640 ; p=.008)$. Additionally, class attendance did not predict GPA. Participants with BMI classifications of overweight or obese had decreased self-efficacy and reduced class attendance than participants with BMIs in the normal weight category. Furthermore, academic selfefficacy significantly predicted GPA $(\beta=.037 ; t=2.442 ; p=.015)$. It was concluded if students who are overweight or obese experience weight stigma or weight discrimination then academic self-efficacy may decrease.

Odlaug et al. (2015) conducted a study to determine if there were relationships between weight and college student's academic achievement and psychiatric disorders. Results were stratified by sex. Participants who self-reported as overweight or obese $(n=$ 492) reported significantly lower GPAs (males, $p=.010$; females, $p<.001$ ) and significantly higher rates of major depressive disorder (males, $p<.001$; females, $p=$ .005) than participants who self-reported as normal weight $(n=1,273)$. This study did not control for any confounding variables (e.g., socioeconomic status) therefore weakening the relationship between BMI and GPA.

\section{Images}

Images are powerful in persuasion. Regardless of the text associated with a message, the message can be viewed as stigmatizing if the image accompanying the text depicts the stigmatized group in a negative manner (Puhl, Luedicke, \& Heuer, 2013). The literature on images of people who are overweight or have obesity has shown many of these images to be negative, perpetuating weight stigma. 
Heuer, McClure, and Puhl (2011) conducted a content analysis of the types of images that five major online news websites choose to use to accompany stories about obesity. For two weeks articles about obesity were collected. Due to the high number of these articles on each website (e.g., 78,200 articles from one new website), the first 500 articles were selected and then included in analysis if the articles about obesity included an image of a person. A total of 441 people in the images were analyzed.

Heuer et al. (2011) developed their own coding tool. Negative characteristics of images included people who were headless, shown from the side or rear angle, and wearing inappropriate fitting clothing. Positive characteristics of images included people who were wearing professional clothes, shown exercising, and portrayed as an expert or advocate. In addition, the researchers developed six criteria to determine if an image was weight stigmatizing (e.g., disproportionately emphasized an overweight/obese person's abdomen or lower body). If an image met one or more of these criteria, it was determined to be a stigmatizing image.

They found $72 \%$ of images to be weight stigmatizing, with $52 \%$ of images of people who are overweight or have obesity to be shown by only their abdomens or only revealing their lower bodies (Heuer et al., 2011). When comparing images of overweight/obese persons ( $\mathrm{N}=287)$ to images of non-overweight images $(\mathrm{N}=119)$, the percentage of overweight/obese persons shown by negative characteristics was higher for each one (e.g., $59 \%$ of overweight/ obese persons were shown "headless," versus $6 \%$ of non-overweight persons). When analyzing for positive characteristics, the percentage of overweight/obese persons being shown positively was always lower than the non- 
overweight persons (e.g., 11\% of overweight/obese persons were wearing professional clothing versus $50 \%$ of non-overweight persons).

Puhl, Luedicke, and Heuer (2013) also conducted research on images used to accompany news articles, finding comparable results. They randomly assigned participants $(\mathrm{N}=1,251)$ to one of three online experimental surveys. Participant's perceptions and reactions to positive (e.g., in kitchen cutting produce) and negative (e.g., sitting on couch eating junk food) images of adults with obesity, with varying gender and race, were assessed via survey questions. Questions included, "This photo makes me dislike obese people," and "When I see photos like this in the news, it makes me angry because they are insulting to obese people" (Puhl et al., 2013, p. 811). Images included a person with obesity who was either a Caucasian female, an African American female, or a Caucasian male. Each image was paired with the same news article about the prevalence of obesity.

Puhl et al. (2013) found participants who viewed negative, weight stigmatizing images of a person with obesity had higher ratings of laziness and dislike toward people with obesity. Participants who viewed positive, non-stereotypical images of a person with obesity had more positive attitudes toward people who are obese. The findings were consistent across gender, while the female African American elicited higher dislike from participants than the Caucasian female. Findings remained consistent when controlling for participants' income, education, race, gender, and BMI.

\section{Summary of Weight Stigma Scholarly Review}

Although there is limited research on weight stigma experienced by college students, the existing research does indicate college students, males and females alike, 
experience this stigma (Puhl \& Brownell, 2006; Trainer et al., 2015). The effects from weight stigmatizing experiences are detrimental to academic self-efficacy (Aimé et al., 2017), self-esteem (Stevens, 2018), and body image (Stevens, 2018). Furthermore, images can unintentionally perpetuate weight stigma. The majority of images of people who are overweight or obese used by online news stories show the person in a negative, stereotypical behavior (Heuer, McClure, \& Puhl, 2011). Additionally, when someone reads articles accompanied by stereotypical images, the images increase feelings of dislike toward people with obesity within the reader (Puhl, Luedicke, \& Heuer, 2013). The researcher was not able to locate any literature regarding images of people and weight stigma at higher education institutions.

\section{Weight Discrimination and Policy}

Dutton et al. (2014) defined weight discrimination as a "person's experience of being treated poorly by others because of his/her weight" (p. 2). In adults, weight discrimination is the fourth most frequent type of reported discrimination after gender, race, and age (Puhl, Andreyeva, \& Brownell, 2008). This type of discrimination occurs across numerous settings including the workplace, higher education, healthcare facilities, and interpersonal relationships (Burmeister et al., 2016; Puhl \& Heuer, 2009; Puhl \& King, 2013). Weight discrimination has contributed to individuals not being promoted (Burmeister et al., 2016; Puhl \& Heuer, 2009), being disfavored in interviews for graduate school (Burmeister et al., 2016), and receiving negligent healthcare treatment (Burmeister et al., 2016).

Regardless of research documenting weight discrimination and the negative effects weight discrimination has on the individuals who experience it, in the United 
States only the state of Michigan and six municipalities have legislation in place prohibiting weight discrimination (Pearl, Puhl, \& Dovidio, 2017). Michigan's legislature enacted this law in 1976 (Michigan Department of Civil Rights, 2019), and since then, no state or national weight anti-discrimination legislation has been passed. Just recently though, the Massachusetts legislatures introduced a bill to prohibit weight discrimination (Puhl, 2019).

There is public support for this legislation (Puhl, Suh, \& Li, 2016). From 2011 to 2015, public support for weight anti-discrimination laws increased from $72.2 \%$ to $80.9 \%$, respectively (Puhl et al., 2016). Research has also shown there are positive psychological effects for individuals with overweight and obesity when weight anti-discrimination legislation is in place (Pearl, Puhl, \& Dovidio, 2017). Although policymaker's advocacy efforts for weight anti-discrimination legislation have not become national law, scholars continue to advocate for policy change.

\section{Policy Analysis}

To reduce weight stigma, several scholars have proposed to implement weightinclusive policies (Pearl, Puhl, \& Dovidio, 2017; Puhl, Suh, \& Li, 2016; O’Reilly \& Sixsmith, 2012; Ramos-Salas et al., 2017). O’Reilly and Sixsmith (2012) conducted discourse and policy analyses on health promotion policies. In the discourse analysis, documents analyzed used the principles of the weight-centered paradigm versus the weight inclusive paradigm with the goal of decreasing rates of obesity. Inflammatory words about obesity were found. These words included "combat," "crisis," and "alarming." In addition, O'Reilly and Sixsmith discovered the majority of claims about weight were either from studies with weak methodology or cited as a statement from a 
health expert. One example provided was using the World Health Organization (WHO) as a reference. The researchers discovered that since WHO is a known health entity, public health officials view their findings as fact, despite many of WHO's "claims are not based on strong scientific evidence" (O’Reilly \& Sixsmith, 2012, p. 101).

From this discourse analysis, a policy analysis was then conducted (O'Reilly \& Sixsmith, 2012). The researchers' policy analysis method aligned with Bardach and Patashnik's (2016) eightfold path for policy analysis. Initially, four policy options were proposed. To inform and provide evidence for the final policy recommendations, 10 participants were interviewed. These participants were chosen due to being stakeholders with knowledge about weight, health promotion policies, and weight stigma.

Additionally, the researchers used the criterion of efficacy, equity, cost, public and political buy-in, and implementation coordination to analyze the proposed policies. The policy recommendations that emerged, in rank-ordering were: (a) create and utilize interventions and programs in weight-neutral, HAES language; (b) provide anti-weight bias training; and (c) develop research data collection, analysis, and evaluation guidelines that use variables beyond just weight, thus reducing dependence on and promotion of the weight-centered health paradigm (O’Reilly \& Sixsmith, 2012).

Utilizing HAES, weight-neutral language in health promotion policies would cost little to nothing to implement, and was viewed as politically feasible and accepted by the community (O’Reilly \& Sixsmith, 2012). To support health promotion policy efforts, health promotion campaigns may be implemented. Previous studies on health promotion campaigns showed many of these campaigns to increase weight bias and have unintended 
weight stigmatizing effects (Pearl, Dovidio, \& Puhl, 2015; Puhl, Luedicke, \& Peterson, 2013; Puhl, Peterson, \& Luedicke, 2013).

A randomized controlled trial (RCT) on public health campaigns found the weight stigmatizing campaigns to be rated as least motivating in addition to lowering participants' self-efficacy to improve health than the non-stigmatizing campaigns (Puhl, Luedicke, \& Peterson, 2013). Participants $(N=1,085)$ were randomly assigned to one of 10 weight stigmatizing campaigns or one of 10 neutral, non-stigmatizing campaigns. Examples of stigmatizing campaign messages were, (a) Don't Drink Yourself Fat, (b) Warning: Chubby Kids, and (c) Thighs on Cheese (Puhl et al., 2013). Examples of nonstigmatizing campaign messages were, (a) Find Healthier Drinks, (b) Raise a Healthy Child, and (c) Three Veggies Thursday (Puhl et al., 2013).

Implementing anti-weight bias training was also determined to be feasible due to research studies showing its effectiveness (McVey, Gusella, Tweed, \& Ferrari, 2009; McVey et al., 2013), having established curriculum available (McVey et al., 2009; O’Reilly \& Sixsmith, 2012), and being accepted politically (O'Reilly \& Sixsmith, 2012). McVey et al., 2009, implemented a web-based anti-weight bias training called The Student Body: Promoting Health at Any Size, to 78 elementary school teachers and 89 public health practitioners. In this RCT, $74.6 \%$ of participants in the intervention group reported the training improved their own body acceptance and 94.6\% reported they would make changes in their school environment based on knowledge learned. On the measurement of efficacy to fight weight bias, the teacher intervention group showed no significant change $(p<.76)$ while the public health practitioner intervention group had a significant positive change $(p<.001)$. 


\section{Summary of Weight Discrimination and Policy}

Although there is public support for weight anti-discrimination policy (Puhl, Suh, \& Li, 2016), only the state of Michigan and six municipalities have laws in place prohibiting weight discrimination (Pearl, Puhl, \& Dovidio, 2017). Scholars advocating for weight anti-discrimination policies have recommended strategies to include weightneutral, HAES language (O’Reilly \& Sixsmith, 2012). A policy analysis concluded implementing this type of language would be cost-effective and politically feasible (O’Reilly \& Sixsmith, 2012). With the prevalence of weight stigma increasing (Andreyeva et al., 2008) and weight discrimination being the fourth most frequently reported type of discrimination (Puhl, Andreyeva, \& Brownell, 2008), more research is warranted to assist in understanding the sources of weight stigma to aid in creating weight inclusive environments in addition to guiding policymakers in this process.

\section{Conclusions}

The HAES paradigm was created as a substitute paradigm to the weight-centered approach used to aid in helping individuals manage their weight (Robison, 1997). There is evidence that the HAES paradigm increases psychosocial and physiological outcomes for people who are overweight or obese (Bacon \& Aphramor, 2011), and in the past six years, this paradigm has embraced a social justice role (ASDAH, 2018). This role includes having individuals and organizations work to eliminate weight bias, weight stigma, and weight discrimination, in addition to accepting and respecting the differences in all people's body shapes and sizes (ASDAH, 2018).

Although there is limited research on weight stigma experienced by college students, the existing research does indicate college students experience this stigma (Puhl 
\& Brownell, 2006; Trainer et al., 2015). The effects from weight stigmatizing experiences are detrimental to academic self-efficacy (Aimé et al., 2017), self-esteem (Stevens, 2018), and body image (Stevens, 2018). Additionally, images can unintentionally perpetuate weight stigma. The majority of images of people who are overweight or obese used by online news stories show the person in a negative, stereotypical behavior (Heuer, McClure, \& Puhl, 2011). Furthermore, when someone reads these articles that are accompanied by stereotypical images, the images increase feelings of dislike toward people with obesity (Puhl, Luedicke, \& Heuer, 2013).

According to Pescosolido and Martin's (2015) definition of stigma, a component of weight stigma is weight discrimination. Although there is public support for weight anti-discrimination policy (Puhl, Suh, \& Li, 2016), only the state of Michigan and six municipalities have laws in place prohibiting weight discrimination (Pearl, Puhl, \& Dovidio, 2017).

Scholars advocating for weight anti-discrimination policies have recommended strategies to include weight-neutral, HAES language (O’Reilly \& Sixsmith, 2012). A policy analysis concluded implementing this type of language would be cost effective and politically feasible (O’Reilly \& Sixsmith, 2012). With the prevalence of weight stigma increasing (Andreyeva et al., 2008) and weight discrimination being the fourth most frequently reported type of discrimination (Puhl, Andreyeva, \& Brownell, 2008), more research is warranted to assist in understanding the sources of weight stigma to aid in creating weight inclusive environments in addition to guiding policymakers in this process. 
SECTION FOUR:

CONTRIBUTION TO PRACTICE 


\section{Plans for Policy Advocacy}

Results and recommendations from this study will be provided to the study site's Committee on Policy Review in the form of an executive summary and policy brief. Membership consists of three faculty members, one whom is committee chair, in addition to the Chair of Faculty Senate, Chair-Elect of Faculty Senate, and Chair of the Faculty Handbook Revision Committee. The director of human resources, a representative from the registrar's office, and a representative from the Student Government Association are also included as committee members. Lastly, members from the strategic apex include the provost and the chief general counsel.

The researcher will be the policy advocate for any new policies or existing policy revisions proposed, and will invest time in gaining support from key constituents for the policy as recommended by Bardach and Patashnik (2016). These constituents include members of the Committee on Policy Review. In addition, the researcher will request being a guest at one of this committee's monthly meetings to share the implications and recommendations from the study. This provides her with a seat at the table, where she can have influence due to being able to be an active participant at the meeting as discussed by Cervero and Wilson (2006). With the provost and chief general counsel being members of the Committee on Policy Review, this would also put the recommendations into the hands of administrators in the study site's strategic apex.

Other university entities that may benefit from having the study's results and recommendations are the Division of Diversity and Inclusion (DDI) and Office for Institutional Equity and Compliance. The chief diversity officer directs the DDI. This division focuses on developing an inclusive campus environment where all students, 
staff, and faculty feel supported. To aid in increasing inclusiveness, one of DDI's roles is to enhance and increase the integration of policy and practices to university stakeholders (MSU, 2021a).

The director of institutional equity and compliance oversees the Office for Institutional Equity and Compliance. This office has a role to ensure the university is compliant with federal, state, and institutional policy to promote a non-discriminatory and harassment-free environment (MSU, 2020). One responsibility includes making policy recommendations. The DDI and Office for Institutional Equity and Compliance may find the results of this study useful. These units could use adaptive leadership principles to implement policy, programs, and practices to increase weight inclusivity at the study site. 


\title{
Becoming "the University of Choice" for Students of All Sizes: Implementing Weight Inclusive Strategies to Promote an Environment of Respect and Enhance Recruitment
}

\author{
Policy Brief for Missouri State University Constituents: Spring 2021 \\ Hillary Roberts, Assistant Professor
}

\begin{abstract}
Viewing Weight Stigma as a Social Justice Issue

Stigma toward people of different races, genders, and religions have been rejected by civilized society. While these stigmas are challenged, weight stigma remains socially acceptable (Puhl \& Heuer, 2010). This policy brief is based on research conducted to examine weight stigma at Missouri State University in Fall 2020. My study included:
\end{abstract}

(1) Analysis of images of people and text used in MSU print materials, social media sites, and webpages. Images were collected by students who self-identified as overweight or obese, and by the researcher.

(2) Analysis of MSU's Policy Library, 2016-2021 Long-Range Plan, and Diversity \& Inclusion Implementation Strategy for weight inclusive and weight stigmatizing body messages.

The American College Health Association (2020) reports $37 \%$ of college students are overweight or obese. Research has found young adults who are obese are less likely to attain a college degree (Fowler-Brown et al., 2010), especially females (Crosnoe, 2007). It is speculated weight stigma contributes to decreased college enrollment and retention. Stigmatizing beliefs and attitudes are maintained and reinforced with exposure to negative messages toward people of larger size. This policy brief will conclude with recommendations for implementation at Missouri State, to extend inclusivity to people of various body sizes.

\section{Students of Larger Size Feel Excluded and Hidden}

Missouri State's vision is to "be the university of choice to develop successful students who excel academically and in ethical leadership, cultural competence, and community engagement" (MSU, 2018). Additionally, MSU values inclusiveness, promoting an environment of respect, and continuous improvement in programs and policies (MSU, 2018). In an analysis of body messages (i.e., images of people and text) captured by MSU student participants, all who self-identified as people of larger size, $41 \%$ of messages were considered weight stigmatizing. These messages either did not include size diversity or were noted as weight stigmatizing because of being placed in a non-prominent place on campus. Additionally, $55 \%$ of the weight stigmatizing messages were located on a MSU Instagram or Twitter feed.

Student participants completed a questionnaire on each photo they took of a 
weight inclusive or weight stigmatizing body message on campus.

"All bodies are good bodies, but we have a stark majority of straight sized and able bodied people constantly being showcased here (Instagram) and that does not exemplify the qualities MSU says they hold" -MSU Student Participant

Here are the findings from the questionnaires student participants completed on each photo they took.

\begin{tabular}{|c|c|}
\hline & $\begin{array}{l}\text { Themes Identified from Students' } \\
\text { Questionnaire Comments }\end{array}$ \\
\hline \multirow{4}{*}{ 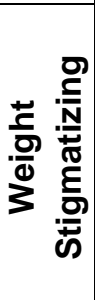 } & Not inclusive of different body sizes \\
\hline & Advertising uses smaller people \\
\hline & Negativity \\
\hline & $\begin{array}{l}\text { Poster placement: Posters hidden } \\
\text { based on body size }\end{array}$ \\
\hline \multirow{4}{*}{ 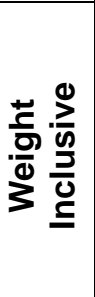 } & Different body sizes \\
\hline & Joy and positivity \\
\hline & $\begin{array}{l}\text { Happy at MSU no matter what } \\
\text { size you are }\end{array}$ \\
\hline & Realistic students \\
\hline
\end{tabular}

"I believe this girl's achievement [was] hidden based on her body."

- MSU Student Participant

Research has suggested the effects from weight stigmatizing experiences are detrimental to academic self-efficacy (Aimé et al., 2017) and self-esteem (Stevens, 2018) of students.

\section{Weight Inclusive Language is Non-Existent in MSU's Policy Library}

While Missouri State has a non-

discrimination policy statement and policies related to diversity and inclusiveness (i.e. employee and student disability accommodation policies, accessibility policy), within these policies, weight discrimination and body size are not addressed. Additionally, body size inclusion and diversity are absent from the 2016-2021 long-range plan and the diversity and inclusion implementation strategy. It is important to note, this is not the exception. In the United States only the state of Michigan and six municipalities have legislation in place prohibiting weight discrimination (Pearl, Puhl, \& Dovidio, 2017). Therefore, non-discrimination policies in higher education institutions in Michigan include not discriminating due to weight (University of Michigan, 2021), but outside of Michigan, higher education policies prohibiting weight discrimination are rare.

Additionally, MSU has faculty and staff diversity composition initiatives. Candidates for these initiatives must have a commitment to diversity. Within the qualifications for these initiatives, diversity related to race, ethnicity, sexual orientation, plus several more categories are listed, but body size diversity is not included.

Lastly, MSU has a performance evaluation appeal policy where employees who believe they have been discriminated against can take action. Discrimination based on body size is not included.

Why does weight stigma matter? Within the context of education, research has found: 
- $32 \%$ of females $(n=2,449)$ experienced weight stigma from a professor (Puhl \& Brownell, 2006).

- $27 \%$ of males $(n=111)$ experienced weight stigma from an educator (Puhl \& Brownell, 2006).

- Weight stigma from college instructors may contribute to decreased academic self-efficacy and decreased GPA in students (Aimé, Villatte, Cyr, \& Marcotte, 2017).

- Participants who self-reported as overweight or obese $(n=492)$ reported significantly lower GPAs and significantly higher rates of major depressive disorder than participants who self-reported as normal weight ( $n=$ 1,273) (Odlaug et al, 2015).

- When weight stigma was perceived as weight discrimination, individuals who experienced it were 2.41 times more likely to develop more than three psychiatric diagnoses (e.g., anxiety disorder, substance disorder) than individuals who did not perceive weight discrimination (Hatzenbuehler, Keyes, \& Hasin, 2009).

\section{Proposed Strategies to Increase Weight Inclusivity at MSU}

Based upon data from my research, the

following strategies are proposed to help

decrease weight stigma and weight

discrimination at Missouri State:

- Adapt imaging in MSU print materials, social media platforms, and webpages to be weight inclusive.

- Add body size to MSU's nondiscrimination policy statement.

- Add discrimination based on body size to the performance evaluation appeal policy.

- Increase use of weight-inclusive language in health promotion policies (O'Reilly \& Sixsmith, 2012).

- Implement anti-weight bias or size diversity trainings for faculty and staff (O'Reilly \& Sixsmith, 2012).
- Implement weight-inclusive health promotion campaigns. It is important to note many health promotion campaigns have increased weight bias and had unintended weight stigmatizing effects (Pearl, Dovidio, \& Puhl, 2015; Puhl, Luedicke, \& Peterson, 2013; Puhl, Peterson, \& Luedicke, 2013).

\section{Policy Recommendation}

$\checkmark$ Adapt imaging in MSU print materials, social media platforms, and webpages to be weight inclusive by adopting a Diversity and Inclusion Image Policy.

\section{Program Recommendation}

$\checkmark \quad$ Include size diversity learning opportunities within the Division for Diversity and Inclusion.

\section{Practice Recommendation}

$\checkmark$ Implement weight-inclusive health promotion campaigns.

\section{How to Achieve Recommendations}

The recommendations presented here have minimal if any planning and implementation costs, in addition to being politically feasible.

Creation of a Diversity and Inclusion Image Policy would provide guidelines for best practices on selecting images of people for MSU print materials, social media platforms, and webpages. Best practices would address not only size diversity but also racial, gender, age, and ability diversity. Regarding size diversity, SELF Magazine's Style Guidelines (Kylstra, 2018) could help guide the criterion.

Implement faculty, staff, and student size diversity training, where the Diversity and Inclusion Image Policy would be discussed. Increasing awareness of the lack of size 
diversity in MSU images would hopefully result in an increased use of images of students of all sizes. This could help with recruitment of students of larger size and promote a respectful environment.

Additionally, within this training, how to use weight-inclusive language would be shared. Furthermore, hanging banners that include images of students who are overweight or obese in prominent locations would be addressed. It is vital students do not feel achievements of students of size are intentionally being hidden because of their body size.

MSU students who are overweight or obese noted feeling excluded and hidden by the non-weight inclusive images they saw on campus and on MSU Instagram and Twitter feeds. MSU can do better! Weight-inclusive health promotion campaigns could be developed and implemented by graduate nutrition and dietetic students as part of their coursework. These campaigns could be marketed through the Student Wellness unit of Magers Health and Wellness Center.

With the prevalence of weight stigma increasing (Andreyeva, Puhl, \& Brownell, 2008) and weight discrimination being the fourth most frequently reported type of discrimination (Puhl, Andreyeva, Brownell, 2008), MSU can make a difference in providing weight inclusive environments for all students by implementing these policy, program, and practice recommendations.

\section{References:}

- $\quad$ Aimé, A., Villatte, A., Cyr, C., \& Marcotte, D. (2017). Can weight predict academic performance in college students? An analysis of college women's self-efficacy, absenteeism, and depressive symptoms as mediators. Journal of American College Health, 65(3), 168-176. doi:10.1080/07448481.2016.1266639

- American College Health Association. (2020). National College Health Assessment III: Reference Group Executive Summary Spring 2020. Silver Spring, MD: American College Health Association.

- $\quad$ Andreyeva, T., Puhl, R. M., \& Brownell, K. D. (2008). Changes in perceived weight discrimination among Americans, 1995-1996 through 2004-2006. Obesity, 16, 1129-1134. doi:10.1038/oby.2008.35

- $\quad$ Benson, R., von Hippel, P. T., \& Lynch, J. L. (2017). Does more education cause lower $\mathrm{BMI}$, or do lower-BMI individuals become more educated? Evidence from the National Longitudinal Survey of Youth 1979. Social Science \& Medicine, 211, 370-377.

- $\quad$ Crosnoe, R. (2007). Gender, obesity, and education. Sociology of Education, 80(3), 241-260.

- Fowler-Brown, A. G., Ngo, L. H., Phillips, R. S., \& Wee, C. C. (2010). Adolescent obesity and future college degree attainment. Obesity, 18(6), 1235-1241.

- Hatzenbuehler, M. L., Keyes, K. M., \& Hasin, D. S. (2009). Associations between perceived weight discrimination and the prevalence of psychiatric disorders in the general population. Obesity, 17(11), 20332039.

- Kylstra, C. (2018, June 25). How should a health brand talk about weight? An evolving style guide. SELF. Retrieved from: https://www.self.com/story/self-weighthealth-style-guide

- $\quad$ Missouri State University. (2018, June 18). About Missouri State mission, vision and values. Retrieved from https://www.missouristate.edu/about/mission -statement.htm

- $\quad$ Odlaug, B. L., Lust, K., Wimmelmann, C. L., Chamberlain, S. R., Mortensen, E. L., Derbyshire, K., Christenson, G., \& Grant, J. E. (2015). Prevalence and correlates of being overweight or obese in college. Psychiatry Research, 227(1), 58-64.

- O'Reilly, C., \& Sixsmith, J. (2012). From theory to policy: Reducing harms associated with the weight-centered health paradigm. Fat Studies, 1(1), 97-113.

- Pearl, R. L., Dovidio, J. F., \& Puhl, R. M. (2015). Visual portrayals of obesity in health media: Promoting exercise without 
perpetuating weight bias. Health Education Research, 30(4), 580-590.

- $\quad$ Pearl, R. L., Puhl, R. M., \& Dovidio, J. F. (2017). Can legislation prohibiting weight discrimination improve psychological wellbeing? A preliminary investigation. Analyses of Social Issue and Public Policy, 17(1), 84104.

- $\quad$ Puhl, R. M., \& Brownell, K. D. (2006). Confronting and coping with weight stigma: An investigation of overweight and obese adults. Obesity, 14(10), 1802-1815.

- Puhl, R. M., \& Heuer, C. A. (2010). Obesity stigma: Important considerations for public health. American Journal of Public Health, 100, 1019-1028.

- $\quad$ Puhl, R. M., Andreyeva, T., \& Brownell, K. D. (2008). Perceptions of weight discrimination: Prevalence and comparison to race and gender discrimination in America. International Journal of Obesity, 32, 9921000. doi:10.2105/AJPH.2009.159491

- Puhl, R., Luedicke, J., \& Peterson, J. L. (2013). Public reactions to obesity-related health campaigns: A randomized controlled trial. American Journal of Preventive Medicine, 45(1), 36-48.

- $\quad$ Puhl, R., Peterson, J. L., \& Luedicke, J. (2013). Fighting obesity or obese persons? Public perceptions of obesity-related health messages. International Journal of Obesity, 37(6), 774-782.

- $\quad$ Stevens, C. (2018). Fat on campus: Fat college students and hyper(in)visible stigma. Sociological Focus, 51(2), 130-149. doi:10.1080/00380237.2017.1368839

- University of Michigan. (2021). University of Michigan nondiscrimination policy. Retrieved from

https://oscr.umich.edu/NondiscriminationPoli cy

This policy brief is based on the research, "Weight Stigma at a University: A Study of Policy, Media, and Student Experiences with Implications for Improving Weight-Inclusivity." Complete study can be requested from HRoberts@missouristate.edu. 
SECTION FIVE:

CONTRIBUTION TO SCHOLARSHIP 


\begin{abstract}
Author's Note
The researcher plans to submit this article to Stigma and Health, an American Psychological Association journal. Articles are not to exceed 20 pages with tables and references being outside of this page limit (American Psychological Association, 2021). Qualitative studies are encouraged, and manuscripts should use APA Style, 7th edition. Additionally, the author chose to use the phrase, people with overweight or obesity, in this article. While that phrase is not used in other sections of the dissertation, it was used here due to it being the preferred language of the Stigma and Health journal.
\end{abstract}




\begin{abstract}
Media images of and text concerning people with overweight and obesity frequently perpetuate weight stigma. This qualitative study explored college student perspectives of body messaging at a public university, and investigated images of people used on university webpages for being weight inclusive or weight stigmatizing body messaging. Body messaging included images of people and text found within university print materials, social media platforms, and webpages. The study engaged a sample of seven participants through photo-elicitation to collect body messages the university used in print materials and social media platforms. Participants then completed a questionnaire about each photo. Additionally, the researchers collected additional images of people from the university webpages. From the 27 participant-generated photos, over a third did not have size diversity or were placed in non-prominent locations on campus.

Participants reported feeling excluded and hidden by the non-weight inclusive messages. Of the 65 images of people collected by the researchers from the university webpages, almost three-fourths did not include size diversity. This study provided evidence that body messaging can contribute to weight stigma and gives suggestions for increasing size diversity of images of people used in university media. Recommendations are provided for university constituents on how to promote weight inclusive environments through policy and programs.
\end{abstract}

Keywords: images, media, messages, photo-elicitation, weight stigma 


\section{Weight Stigma in University Media:}

\section{Analysis of Body Messages from Images and Student Experiences}

Images are powerful in persuasion and are prolific in modern civilization. This bombardment includes images of people on multiple mediums including social media, print and broadcast media, and websites. Data on images of people with overweight or obesity has shown many of these images to be negative, perpetuating weight stigma (Heuer, McClure, \& Puhl, 2011; Puhl, Luedicke, \& Heuer, 2013). Additionally, previous studies on health promotion campaigns showed many of these campaigns to increase weight bias, having unintended weight stigmatizing effects (Pearl, Dovidio, \& Puhl, 2015; Puhl, Luedicke, \& Peterson, 2013; Puhl, Peterson, \& Luedicke, 2013.)

Furthermore, Stevens (2018) found documents on a college campus contributed to feelings of weight stigma. While data exists on images of people who have overweight or obesity used by online news stories (Heuer, McClure, \& Puhl, 2011), there is no data regarding images of people used in higher education institutions and how students may experience weight stigma from these images. Additionally, limited data exists on how written documents contribute to weight stigma at higher education institutions.

Stevens (2018) found considerable amounts of weight stigma in her exploratory study of qualitative data collected from 14 students at two midwestern state universities. She stated, "fat students are bombarded with antiobesity information around campus" (p. 136). The consistent rhetoric to lose weight contributes to individuals having lower selfesteem and poor body image (Stevens, 2018; Wellman, Araiza, Newell, \& McCoy, 2018). Although Stevens's study did not include document collection, her participants noted that documents on campus (e.g., campus newspaper, signs in bathroom stalls, 
materials at student health center) triggered feelings of weight stigma. Thorel et al. (2020) investigated the extent of blame, distrust, and desire for social distance amongst college and other adult participants in response to vignette targets comparing obesity, eating disorders, major depression, and controls. Obesity and binge eating targets solicited the most blame, held the most responsible, for their situations.

Within the context of education, Puhl and Brownell (2006) discovered 32\% of adult females $(n=2,449)$ experienced weight stigma from a teacher or professor, with $21 \%$ experiencing this stigma from a teacher or professor more than once. Male students also experience weight stigma within education. In a sample size of 111 males, $27 \%$ had experienced weight stigma from an educator (Puhl \& Brownell, 2006). Additionally, researchers using data from the National Longitudinal Study of Adolescent Health reported females with obesity were $50 \%$ less likely to attend college than females who were not obese (Crosnoe, 2007). Benson, von Hippel, and Lynch (2017) found similar findings using data from the National Longitudinal Survey of Youth 1979. Females with overweight or obesity were less likely to attain a college degree than females with normal weight status (Benson, von Hippel, \& Lynch, 2017). Fowler-Brown, Ngo, Phillips, \& Wee (2010) also found adolescents with obesity were less likely to attain a college degree than adolescents with normal weight status (10\% vs. $24 \%)$. The mechanism underlying these associations is not known. It is speculated though that weight stigma contributes to these lower rates of achievement (Fowler-Brown et al., 2010).

The researchers define weight stigma as the devaluing of social identify due to an individual's body size. This stigma is pervasive (Puhl \& Heuer, 2010; Ramos-Salas et al., 2017), and the consequences of weight stigma are severe, including having a negative 
effect on the psychological and physical health of individuals who experience it (Gloor \& Puhl, 2016; Puhl \& Heuer, 2009). In the limited studies on the consequences of weight stigma within higher education, data suggests weight stigmatizing experiences from college instructors may contribute to decreased academic achievement and self-efficacy in students (Aimé, Villatte, Cyr, \& Marcotte, 2017). Crandall (1995) followed by Incollingo Rodriguez et al. (2019) found weight stigma undermined academic achievement, including with less financial support. Twenty-five years apart these studies found body mass index to be lower in first year college students than a non-college representative sample. Additionally, thinner students received more parental financial support, suggesting lifelong consequences may be related to weight stigma in college aged persons. Students with overweight or obesity had reduced self-efficacy and reduced class attendance than students who did not have overweight or obesity (Aimé et al., 2017). Furthermore, academic self-efficacy significantly predicted grade point average. It was concluded if students with overweight or obesity experienced weight stigma or weight discrimination then academic self-efficacy may decrease.

Weight stigma is widespread in North American society (Puhl \& Heuer, 2010; Ramos-Salas et al., 2017), yet little research exists on the sources and modality leading to weight stigma on college campuses. With $37 \%$ of college students having overweight or obesity (American College Health Association, 2020), research is needed to investigate how these students experience weight stigma within higher education. Therefore, the purpose of this current study was twofold. First, through photo-elicitation and questionnaires on their experiences of the photos, the researchers explored college student perspectives of images and text used in body messaging at a midwestern, master's 
comprehensive public university. Researchers sought student perspectives and feelings of how they found the body messages to be weight stigmatizing or weight inclusive. Secondly, the researchers investigated images of people used on the university's webpages for weight inclusive and weight stigmatizing images of people. This content analysis provided information on how images of people contribute to, or detract from, providing a weight inclusive environment in a university setting.

\section{Method}

\section{Participants and Procedures}

Participants consisted of students enrolled in any university-level course at a midwestern, master's comprehensive public university, who were 18 years of age or older and self-identified as a person with overweight or obesity. Individuals were excluded from participating if they self-reported as a person with normal weight or underweight body status. Participants were recruited via the university student wellness online newsletter, electronic advertisements posted by political science and biomedical science course instructors willing to assist with recruitment, and flyers posted in an academic building. Students interested in participating reviewed and completed an online consent form prior to participation. The study received institutional review board approval by the university before participant recruitment.

A total of 17 students completed the consent form and then the sociodemographic survey. After excluding those who self-identified as a person with normal weight or underweight, the number of participants included was eight; however, only seven of the eight participants who met the study criterion, participated in photo-elicitation $(n=7)$. The average age of these seven participants was $20.7(S D=1.70)$ with $57.1 \%$ identifying 
as male and $42.9 \%$ identifying as female. The majority of participants identified as White (71.4\%), whereas $14.3 \%$ identified as Black and $14.3 \%$ identified as Latino/a. Additionally, $71.4 \%$ of participants identified as overweight, and $29.6 \%$ identified as obese. Participants declared academic majors were from three of the university's seven different academic colleges, including arts and letters, health and human services, and natural and applied sciences.

\section{Measures}

Sociodemographic information. Participants responded to a questionnaire requesting their sociodemographic information. Respondents reported their gender, age in years, race, body size with which they identified, and academic college of their declared major.

Photo-elicitation and experiences. Photo-elicitation and experiences of the photos via questionnaire were requested of the participants. Merriam and Tisdell (2016) referred to this data collection technique as photovoice.

Photo-elicitation. Participants were asked to take five or more photographs over a two-week period, capturing examples of weight inclusive and weight stigmatizing university body messaging. This aligned with the researcher's guide for photo-elicitation from Bates, McCann, Kaye, and Taylor (2017). Data collection occurred asynchronously, from October 6, 2020, to November 15, 2020. Body messaging was defined as images of people and text found on the university's website or social media platforms. The messaging had to be distributed by a university entity. For example, university banners highlighting students' accomplishments, university department marketing brochures, university social media sites, and university student organization 
materials met the body messaging definition, while an advertisement for a job off-campus did not. Similarly, body messaging in the dining centers did not qualify as those were managed by a contracted agency, not the university directly.

Participants used their own electronic device (i.e., smartphone, tablet) to take a photo and then emailed the photo to the researcher who then uploaded the photo to a secure online server. Every time a photo was emailed to the researcher, the participant received an email message providing the online survey link to the questionnaire about each photographed item.

Photo-elicitation questionnaire. This questionnaire was developed in Qualtrics, an online survey software. Participants stated what experiences, i.e., feelings or thoughts, the photo elicited in them; whether they found the message to be weight inclusive or weight stigmatizing; why they chose to take the photo; and the location of the photographed item.

Document collection online. Following traditional qualitative research methods, the researchers were instruments for data collection (Merriam \& Tisdell, 2016). The lead researcher collected visual documents of images of people displayed on the university's webpages. Screenshots of images were captured on the university's homepage, in addition to screenshots of images that were one-click away from the homepage, and screenshots of images on the university diversity webpage. Furthermore, content on the university's homepage changes periodically. Due to this, images on the homepage were collected daily for a two-week period; from September 1, 2020, through September 15, 2020. 


\section{Data Analytic Plan}

Constant comparative analysis. Constant comparative analysis (Krueger \& Casey, 2015; Merriam \& Tisdell, 2016) was used to analyze the completed photoelicitation questionnaires. Completed photo-elicitation questionnaires were coded using an inductive process with in vivo coding (Creswell, 2016) being implemented. To increase verifiability, the researcher conducted an audit trail (Merriam \& Tisdell, 2016) to record this process. In the log the researcher included each collected photo, recording which participant took the photo, and the date each photo was captured. In addition, the photo-elicitation questionnaire completed by participants on each photo was included. All data was de-identified. Data was referred to as participant (P), photo (PH), and questionnaire (Q), and recorded as such. For example, in the audit trial log, participant one was P1 and the fifth photo they took was P1-PH5. When referring to the completed questionnaire for this photo, it was recorded as P1-PH5-Q5.

Qualitative content analysis. Images of people collected from the university's webpages were analyzed via qualitative content analysis (Kuckartz, 2014). The content analysis followed Elo and Kyngäs's (2008) deductive approach. A priori codes were developed and integrated into the body messaging analysis coding protocol. These a priori codes were developed using language from: (a) the five principles of the Health at Every Size (HAES $®)$ paradigm (ASDAH, 2018; Health at Every Size and HAES are registered trademarks of the Association for Size Diversity and Health and used with permission); (b) tenets of the weight-centered health paradigm outlined by O'Hara and Taylor (2018); (c) values and principles of the HAES paradigm outlined by O'Hara and Taylor (2018); (d) coding protocol of how people who are overweight or obese are portrayed in online news report developed by Heuer, McClure, and Puhl (2011); and (e) 
SELF magazine's style guidelines (Kylstra, 2018) for words and imagery related to weight. The protocol included the weight inclusive a priori codes of: (a) portrays person who is overweight or obese wearing professional clothes; (b) portrays person who is overweight or obese as an expert or advocate, and (c) portrays person who is overweight or obese as a full person. The protocol included the weight stigmatizing a priori codes of: (a) portrays person who is overweight or obese with inappropriately fitting clothes, (b) person who is overweight or obese is not shown as a full person, and (c) portrays person who is overweight or obese eating or drinking unhealthy food or drink. Images were reviewed by the lead researcher and coded into the identified category the content corresponded within the body messaging analysis coding protocol.

Lead researcher's positionality. The researcher is a dissertation student and registered dietitian nutritionist (RDN) with training in nutrition and dietetics. While she self-identifies as a small person and was an outsider with her participants' body sizes, in her career as an RDN she has developed deep empathy with people in larger bodies when she provided nutrition counseling to numerous clients who had deep resentment of their body size. She believes a person's health cannot be defined by their body mass index, although many people believe this to be truth as it is what modern-day society tells them and reinforces (O'Reilly \& Sixsmith, 2012). The researcher is now an assistant professor at a public university and uses the HAES paradigm as a guiding perspective when instructing students. The researcher coded all data, and continuously used reflexivity (Merriam \& Tisdell, 2016) throughout the coding process. 


\section{Results}

\section{Student Experiences of University Body Messages}

Total number of body message photos collected by participants was 28 , each accompanied by a questionnaire articulating the students' experiences. One photo and associated questionnaire were excluded due to the questionnaire data not aligning with the photo. Of the 27 photos analyzed, 25 of these were of images of people, and two were of text. The two photos of text were of the same sign advertising a body positivity discussion. Participants identified the majority of body messages (59.3\%) as weight inclusive with $40.7 \%$ identified as weight stigmatizing. The weight stigmatizing messages either did not include size diversity or the messages were noted as weight stigmatizing because of placement on campus (e.g., located on the side of a nonprominent building instead of the front of a prominent building). Additionally, $55 \%$ of the weight stigmatizing messages were located on the university's Instagram or Twitter feeds. One participant commented, “All bodies are good bodies, but we have a stark majority of straight sized and able bodied people constantly being showcased here [Instagram] and that does not exemplify the qualities [the university] says they hold." Participants categorized $59.3 \%$ of the body messages as weight inclusive. Of these images, $68.8 \%$ were on the university's social media sites with $31.2 \%$ found on the university campus. Table 1 provides the weight inclusive themes identified from the constant comparative analysis of the open-ended responses participants completed in the photo-elicitation questionnaires. Themes are listed in order from highest frequency to lowest frequency. The most frequent identified weight inclusive theme was different body sizes. Participants' responses included words such as "supporting all body types," 
and "[image] involves all shapes and sizes of weight." Those responses were paired with feelings of joy, positivity, comfortableness, and warmness. The joy/positivity theme was affective in that these feelings were internalized by the participants, whereas the happy at [university] no matter what size you are theme was cognitive. Happiness at the university was what was comprehended by the participant when they viewed the body message, not what was felt by the participant.

Table 1

Weight Inclusive Findings from Photo-Elicitation Questionnaires

\begin{tabular}{|c|c|}
\hline Weight Inclusive Theme & Questionnaire Responses \\
\hline Different body sizes & $\begin{array}{l}\text { "Supporting all body types.” (ID\# P1-PH1-Q1) } \\
\text { "There were women of different shapes and } \\
\text { sizes.” (ID \#P2-PH1-Q1) } \\
\text { “...different body shapes in this photo." (ID\# } \\
\text { P7-PH2-Q2) }\end{array}$ \\
\hline Joy/positivity & $\begin{array}{l}\text { “Positive feelings.” (ID\# P1-PH1-Q1) } \\
\text { “This photo brings me joy.” (ID\# P3-PH4-Q4) }\end{array}$ \\
\hline $\begin{array}{l}\text { Happy at [university] no matter what } \\
\text { size you are }\end{array}$ & $\begin{array}{l}\text { “...everyone can have fun at [university] no } \\
\text { matter what size.” (ID\# P3-PH2-Q2) } \\
\text { “...everyone is happy no matter what they look } \\
\text { like.” (ID\# P5-PH2-Q2) }\end{array}$ \\
\hline Realistic students & $\begin{array}{l}\text { "...someone you would realistically see around } \\
\text { campus." (ID\# P1-PH4-Q4) }\end{array}$ \\
\hline
\end{tabular}


Table 2 provides the weight stigmatizing themes identified from the constant comparative analysis of the photo-elicitation questionnaires' open-ended responses. Themes are listed from highest frequency participant responses to lowest frequency, with the first two themes having the same amount of frequency. These two most frequent identified themes were not inclusive of different body sizes and advertising uses smaller people. Participants' responses discussed non-inclusiveness of body types by using words such as, "not having diverse body shapes and sizes," "lack of diversity in body types," and "only shows skinny people." The responses were combined with feelings of unhappiness and negativity by the participant. Additionally, participants' responses pertaining to advertising as being biased included comments discussing campus advertising as including only smaller people to make items look more appealing, in addition to coupling being smaller with "better looking." This advertising referred to obtaining scholarships, jobs, apparel being sold on campus, entrance into degree programs such as the fine arts, and choosing an apartment.

Table 2

Weight Stigmatizing Findings from Photo-Elicitation Questionnaires

\begin{tabular}{ll}
\multicolumn{1}{c}{ Weight Stigmatizing Theme } & \multicolumn{1}{c}{ Questionnaire Responses } \\
\hline Not inclusive of different body & “...only skinny people are showing school \\
sizes & spirit.” (ID\# P3-PH5-Q5) \\
& “...lack of diversity in body types demonstrates \\
& how campus culture is by design not inclusive \\
& and thoughtlessly reinforced and celebrated.” \\
& (ID\# P8-PH1-Q1)
\end{tabular}




\begin{tabular}{ll}
\hline Advertising uses smaller people & “...a lot of their (university Instagram page) \\
& advertising is blatantly bias [sic] towards better \\
& looking and slimmer people.” (ID\# P5-PH5-Q5) \\
& "I've seen a lot of things on campus that \\
& advertise things with smaller people including \\
& this picture.” (ID\# P1-PH5-Q5) \\
& "Negative feelings...” (ID\# P1-PH3-Q3) \\
Negativity & "Unhappy” (ID\# P5-PH1-Q1) \\
Poster placement: Posters hidden & hidden believe this girl’s achievement were [sic] \\
based on body size & hidden her body.” (ID\# P5-PH3-Q3)
\end{tabular}

\section{University's Website Images}

Table 3 provides data on criterion the images of people met to be coded as weight inclusive.

Homepage. Of the 10 images of people collected from the university's homepage, only one was coded as weight inclusive, i.e., including a person with overweight or obesity. While $90 \%$ of the images did not include size diversity, the images did not portray anyone with overweight or obesity in a stigmatizing manner. The university's homepage was not modified during the document collection period.

One-click away from homepage. There were 43 images of people collected one-click away from the university homepage. Aligning with the image analysis on the homepage, while the majority $(76.7 \%)$ did not include size diversity, no images portrayed 
anyone with overweight or obesity in a stigmatizing manner. Ten of the images $(23.3 \%)$ were coded weight inclusive.

Diversity webpage. There were 12 images of people collected on the university's diversity webpage. Of these images, seven (58.3\%) were coded weight inclusive. None of the images were weight stigmatizing.

Table 3

Findings from Weight Inclusive Images of People Collected on University Webpages

\begin{tabular}{lcccccc}
\hline $\begin{array}{l}\text { Webpage } \\
\text { location }\end{array}$ & $\begin{array}{c}\text { Total } \\
\text { number } \\
\text { of images }\end{array}$ & $\begin{array}{c}\text { Weight } \\
\text { inclusive } \\
\text { images }\end{array}$ & $\begin{array}{c}\text { Person is } \\
\text { wearing } \\
\text { profession } \\
\text {-al clothes }\end{array}$ & $\begin{array}{c}\text { Person is } \\
\text { portrayed } \\
\text { as a full } \\
\text { person }\end{array}$ & $\begin{array}{c}\text { Person is } \\
\text { engaged } \\
\text { in } \\
\text { physical } \\
\text { activity }\end{array}$ & $\begin{array}{c}\text { Person is } \\
\text { portrayed } \\
\text { as an } \\
\text { expert }\end{array}$ \\
\hline $\begin{array}{l}\text { Homepage } \\
\begin{array}{l}\text { One-click } \\
\text { away from } \\
\text { homepage }\end{array}\end{array}$ & 10 & 1 & 1 & 1 & 0 & 0 \\
$\begin{array}{l}\text { Diversity } \\
\text { webpage }\end{array}$ & 12 & 10 & 6 & 7 & 2 & 0 \\
\hline Total & $\mathbf{6 5}$ & $\mathbf{1 8}$ & $\mathbf{1 0}$ & $\mathbf{1 4}$ & $\mathbf{2}$ & $\mathbf{1}$ \\
\hline
\end{tabular}

Note. Person refers to a person with overweight or obesity.

\section{Discussion}

From the university campus and the university's social media pages, participants collected 27 body messages, 25 images of people, and two of text. The majority (59.3\%) of those messages were categorized as weight inclusive. The strongest weight inclusive theme associated with the body messages were different body sizes. Feelings and thoughts associated with the weight inclusive messages were joy and positivity. Alternatively, $40.7 \%$ of body messages were identified to be weight stigmatizing. Strongest weight stigmatizing themes were being non-inclusive of different body sizes 
and advertising using smaller people. Negative thoughts and feelings were associated with these messages. These preliminary findings contribute to filling a gap in the literature on what weight inclusive and weight stigmatizing body messages college students with overweight or obesity experience on a university campus.

Data analysis of the 65 images of people collected from the university webpages showed almost three-fourths not portraying size diversity with just over one-fourth being weight inclusive. This data collection method did reach saturation. The university did not have any weight stigmatizing images that negatively portrayed people with overweight or obesity, yet at the same time the majority of images of people were not size diverse. These findings are in contrast to a study of images of people used in online news articles about obesity (Heuer, McClure, \& Puhl, 2011). Heuer et al. (2011) found $72 \%$ of the 441 images of people analyzed to be weight stigmatizing (e.g., disproportionately emphasizing the abdomen or lower body of a person with overweight or obesity). Despite this study's findings not discovering any images of people with overweight or obesity being negatively portrayed, it is speculated prospective and current students with overweight or obesity may choose not to attend a university where they do not feel represented by the images they see on the university webpages.

In summary, in this sample of college students with overweight and obesity, of the photographs they took of body messages found on a university campus and associated social media sites, more than half were categorized as weight inclusive, while more than a third of the messages were categorized as weight stigmatizing. Body messages were weight stigmatizing due to the messages not having size diversity or due to being placed in a non-prominent location on campus, not due to a person with overweight or obesity 
shown in a weight stigmatizing manner (e.g., disproportionately emphasizing the abdomen or lower body, or being shown with inappropriately fitting clothes). Of these weight stigmatizing body messages, a little more than half were located on the university's Instagram or Twitter feeds. A surprising finding from this study was how the location of messages was perceived as weight stigmatizing. To the researchers' knowledge, this has not been documented in the literature. Students felt excluded and hidden by these non-weight inclusive messages, whereas the weight inclusive messages led students to feel joyful, happy, and included.

Ninety percent of young adults (18-29 years old) use at least one social media site, with 79\% using Facebook, 67\% using Instagram, and 38\% using Twitter (Pew Research Center, 2019). It is speculated using images of people which do not include size diversity in university messaging, including social media sites, may contribute to decreased recruitment of adolescents with overweight or obesity. Additionally, this contributes to increased perceived weight stigma by students with overweight or obesity. Future studies on how images of people may contribute to student recruitment and retention are warranted.

In addition, future studies on body messaging text are warranted. While participants in this study could take photos of images of people and body messaging text, only two photos of the same text were collected. Previous studies have shown positive psychosocial outcomes, e.g., self-esteem and body image, when weight-neutral, HAES® language is used (Bacon \& Aphramor, 2011). Conducting a study about student experiences of weight stigmatizing and weight inclusive text would provide new knowledge on how body messaging text affects the college student experience. 
While study strengths included a racially diverse sample of females and males with overweight or obesity, this study is limited by having participants self-identify as overweight or obesity. Studies have found mixed data regarding reliability of selfperceived weight status (Chang \& Christakis, 2003; Han et al., 2019; Pursey, Burrows, Stanwell, \& Collins, 2014). However, an accurate weight status was not essential to gain reliable data in this study. Instead, how participants perceived themselves when compared to images viewed on campus or on a university social media platform was an essential component and met. Additionally, weight stigma also exists for people who are underweight (Puhl, Himmelstein, \& Quinn, 2018). With this study not including participants who self-identify as underweight, the data is not generalizable to this population.

Another limitation was the photo-elicitation of body messages and experiences of the photos via questionnaire did not reach saturation. Thus, the findings from the student experiences of body messages through photo-elicitation can only be interpreted as preliminary. The sample size of seven participants was partly due to coronavirus disease 2019 (COVID-19) where the researcher was not permitted to recruit in person.

Furthermore, students experienced more stressors due to COVID-19 in the recruitment period, and it is assumed students did not have the time to commit to volunteer to be a participant. Lastly, the researcher found limited criteria to use to categorize images of people and text as weight-inclusive or weight-stigmatizing. Therefore, the researcher developed her own protocol for the analysis of images of people and text. This protocol has not been tested for reliability and validity. 
Findings from this present study may help guide higher education institutions in creating policies regarding inclusive images of people of different sizes. Research has suggested the effects from weight stigmatizing experiences may be detrimental to academic achievement as concerns the self-efficacy (Aimé et al., 2017), self-esteem (Stevens, 2018), and financial support (Crandall, 1995; Incollingo Rodriguez et al., 2019) associated with achievement. This study's results included participants feeling excluded and hidden by non-weight inclusive body messages. Higher education constituents are encouraged to consider adopting an image policy, providing guidelines for best practices on selecting images of people for university webpages, social media platforms, and print materials. It is recommended for best practices to address size diversity in addition to racial, gender, age, and ability diversity.

Additionally, increasing awareness of non-inclusive body messages contributing to weight stigma for college students is needed. To increase this awareness, scholars have recommended strategies to include weight-neutral, HAES® language (O'Reilly \& Sixsmith, 2012). A policy analysis concluded implementing this type of language would be cost-effective and politically feasible (O'Reilly \& Sixsmith, 2012). Higher education constituents are encouraged to consider implementing weight-neutral language in health promotion policies in addition to providing body size diversity workshops for faculty, staff, and students. In these workshops, unbiased language e.g., HAES® language, could be shared and practiced. Also, the importance of including images of people of all shapes and sizes on university webpages, social media platforms, and print materials should be discussed, as well as location of images used on campus. 
Furthermore, to support health promotion policy efforts, weight-inclusive health promotion campaigns may be implemented. Previous studies on health promotion campaigns showed many of these campaigns to increase weight bias, having unintended weight stigmatizing effects (Pearl, Dovidio, \& Puhl, 2015; Puhl, Luedicke, \& Peterson, 2013; Puhl, Peterson, \& Luedicke, 2013). It is essential health promotion campaigns use unbiased language such as that supported by HAES®.

In conclusion, researchers are encouraged to continue investigating sources and modalities of weight stigma for college students and how to reduce these through policies and programs. This study informs higher education institution constituents of how university body messaging can be used to promote weight inclusive environments. Constituents are encouraged to consider one or more of the following strategies: (a) adopt a diversity and inclusion image policy, (b) implement weight-neutral language in health promotion policies, (c) provide body size diversity workshops, and (d) implement weight-inclusive health promotion campaigns. These strategies will aid in decreasing weight stigma, possibly increasing recruitment, retention, and graduation for students with overweight or obesity. 


\section{References}

Aimé, A., Villatte, A., Cyr, C., \& Marcotte, D. (2017). Can weight predict academic performance in college students? An analysis of college women's self-efficacy, absenteeism, and depressive symptoms as mediators. Journal of American College Health, 65(3), 168-176. doi:10.1080/07448481.2016.1266639

American College Health Association. (2020). National College Health Assessment III: Reference Group Executive Summary Spring 2020. Silver Spring, MD: American College Health Association.

Association for Size Diversity and Health. (ASDAH). (2018). HAES principles. Retrieved from https://www.sizediversityandhealth.org/content.asp?id=76

Bacon, L., \& Aphramor, L. (2011). Weight science: Evaluating the evidence for a paradigm shift. Nutrition Journal, 10(9) 1-13. doi:10.1186/1475-2891-10-9

Bates, E. A., McCann, J. J., Kaye, L. K., \& Taylor, J. C. (2017). “Beyond words”: A researcher's guide to using photo elicitation in psychology. Qualitative Research in Psychology, 14(4), 459-481.

Benson, R., von Hippel, P. T., \& Lynch, J. L. (2017). Does more education cause lower BMI, or do lower-BMI individuals become more educated? Evidence from the National Longitudinal Survey of Youth 1979. Social Science \& Medicine, 211, 370-377.

Chang, V. W. \& Christakis, N. A. (2003). Self-perception of weight appropriateness in the United States. American Journal of Preventive Medicine, 24(4), 332-339. doi: 10.1016/S0749-3797(03)00020-5

Crandall, C. S. (1995). Do parents discriminate against their heavyweight daughters? 
Personality and Social Psychology Bulletin, 21, 724-735.

doi:10.1177/0146167295217007

Creswell, J. W. (2016). 30 essential skills for the qualitative researcher. Los Angeles, CA: Sage.

Crosnoe, R. (2007). Gender, obesity, and education. Sociology of Education, 80(3), 241260.

Elo, S., \& Kyngäs, H. (2008). The qualitative content analysis process. Journal of Advanced Nursing, 62(1), 107-115. doi:10.1111/j.1365-2648.2007.04569.x

Fowler-Brown, A. G., Ngo, L. H., Phillips, R. S., \& Wee, C. C. (2010). Adolescent obesity and future college degree attainment. Obesity, 18(6), 1235-1241.

Gloor, J. L., \& Puhl, R. M. (2016). Empathy and perspective-taking: Examination and comparison of strategies to reduce weight stigma. Stigma and Health, 1(4), 269279. doi: $10.1037 / \mathrm{sah} 0000030$

Han, L., Duan, S., Qi, L. You, D., Zeng, F., Feng, X., \& Astell-Burt, T. (2019). Trends in self-perceived weight status, weight loss attempts, and weight loss strategies among adults in the United States, 1999-2016. JAMA Network Open, 2(11), 1-18. doi: 10.1001/jamanetworkopen.2019.15219

Heuer, C. A., McClure, K. J., \& Puhl, R. M. (2011). Obesity stigma in online news: A visual content analysis. Journal of Health Communication, 16, 976-987.

Incollingo Rodriguez, A. C., White, M. L, Standen, E. C., Mann, T., Wells, C. R., \& Tomiyama, A.J. (2019). Body mass index and educational inequality: An update of Crandall (1995). Stigma and Health, 4(3), 357-363.

Krueger, R. A., \& Casey, M. A. (2015). Focus groups: A practical guide for applied 
research (5th ed.). Los Angeles, CA: Sage.

Kuckartz, U. (2014). Qualitative text analysis: A guide to methods, practice \& using software. Los Angeles, CA: Sage.

Kylstra, C. (2018, June 25). How should a health brand talk about weight? An evolving style guide. SELF. Retrieved from: https://www.self.com/story/self-weighthealth-style-guide

Merriam, S. B., \& Tisdell, E. J. (2016). Qualitative research: A guide to design and implementation (4th ed.). San Francisco, CA: Jossey-Bass.

O'Hara, L., \& Taylor, J. (2018). What's wrong with the 'war on obesity?' A narrative review of the weight-centered health paradigm and development of the $3 \mathrm{C}$ framework to build critical competency for a paradigm shift. SAGE Open, 8(2), 128.

O’Reilly, C., \& Sixsmith, J. (2012). From theory to policy: Reducing harms associated with the weight-centered health paradigm. Fat Studies, 1(1), 97-113.

Pearl, R. L., Dovidio, J. F., \& Puhl, R. M. (2015). Visual portrayals of obesity in health media: Promoting exercise without perpetuating weight bias. Health Education Research, 30(4), 580-590.

Pearl, R. L., Puhl, R. M., \& Dovidio, J. F. (2017). Can legislation prohibiting weight discrimination improve psychological well-being? A preliminary investigation. Analyses of Social Issue and Public Policy, 17(1), 84-104.

Pew Research Center. (2019, June 12). Social media fact sheet. Retrieved from https://www.pewresearch.org/internet/fact-sheet/social-media/

Puhl, R. M., \& Brownell, K. D. (2006). Confronting and coping with weight stigma: An 
investigation of overweight and obese adults. Obesity, 14(10), 1802-1815.

Puhl, R. M., \& Heuer, C. A. (2009). The stigma of obesity: A review and update. Obesity, $17(5), 941-964$.

Puhl, R. M., \& Heuer, C. A. (2010). Obesity stigma: Important considerations for public health. American Journal of Public Health, 100, 1019-1028. doi:10.2105/AJPH.2009.159491

Puhl, R. M., Himmelstein, M. S. \& Quinn, D. M. (2018). Internalzing weight stigma: Prevalence and sociodemographic considerations in US adults. Obesity, 26(1), 167-75.

Puhl, R. M., Luedicke, J., \& Heuer, C. A. (2013). The stigmatizing effect of visual media portrayals of obese persons on public attitudes: Does race or gender matter? Journal of Health Communication, 18, 805-826.

Puhl, R., Luedicke, J., \& Peterson, J. L. (2013). Public reactions to obesity-related health campaigns: A randomized controlled trial. American Journal of Preventive Medicine, 45(1), 36-48

Puhl, R., Peterson, J. L., \& Luedicke, J. (2013). Fighting obesity or obese persons? Public perceptions of obesity-related health messages. International Journal of Obesity, 37(6), 774-782.

Pursey, K., Burrows, T. L, Stanwell, P., \& Collins, C. E. (2014). How accurate is webbased self-reported height, weight, and body mass index in young adults? Journal of Medical Internet Research, 16(1), 1-9. doi: 10.2196/jmir.2909

O’Reilly, C., \& Sixsmith, J. (2012). From theory to policy: Reducing harms associated with the weight-centered health paradigm. Fat Studies, 1(1), 97-113. 
Ramos-Salas, X., Alberga, A. S., Cameron, E., Estey, L., Forhan, M., Kirk, S. F. L., Russell-Mayhew, S., \& Sharma, A. M. (2017). Addressing weight bias and discrimination: Moving beyond raising awarenss to creating change. Obesity Reviews, 18, 1323-1335.

Stevens, C. (2018). Fat on campus: Fat college students and hyper(in)visible stigma. Sociological Focus, 51(2), 130-149. doi:10.1080/00380237.2017.1368839

Thorel, N., Thorel, E., Tuschen-Caffier, B. (2020). Differential stigmatization in the context of eating disorders: Less blame might come at the price of greater social rejection. Stigma and Health. Advance online publication. doi: $10.1037 / \mathrm{sah} 0000274$.

Wellman, J. D., Araiza, A. M., Newell, E. E., \& McCoy, S. K. (2018). Weight stigma facilitates unhealthy eating and weight gain via fear of fat. Stigma and Health, 3(3), 186-194. doi:10.1037/sah0000088 
SECTION SIX:

SCHOLARLY PRACTITIONER REFLECTION 


\section{Scholarly Practitioner Reflection}

The University of Missouri Statewide Cooperative Doctorate of Education (EdD) Program in Educational Leadership has been one of the most difficult yet most transformative experiences of my life. Upon completing all program requirements, except for the dissertation, I was fortunate to meet the qualifications to apply for a tenuretrack position at a four-year public university. I was offered and accepted this position, thus beginning a new journey where I have begun implementing knowledge gained from this EdD program. The dissertation process has increased not only my research knowledge and skills, but also leadership traits such as connectedness and resiliency. This reflection discusses how the dissertation process has influenced my practice as an educational leader and as a scholar, concluding with a summary of the reflection.

\section{Influence on Practice as an Educational Leader}

The dissertation influenced my practice as an educational leader in two distinct ways: increased connectedness and increased resiliency. Both items align with characteristics of an authentic leader (Northouse, 2016), a type of leadership practice I strive to continue to develop. The dissertation process helped me to recognize ways to increase connectedness with others in addition to strategies to increase resiliency.

\section{Connectedness}

While there is not an official definition for authentic leadership (Northouse, 2016), authentic leadership characteristics include compassion, consistency, passion, and connection with others (George, Sims, McLean, \& Mayer, 2007). Connection with others is the characteristic I strive to make a consciousness effort to enhance. As a natural introvert, connection with others is more likely to occur for me when there is a 
problem to be solved. Within the dissertation process, this connection came from having a dissertation committee and problem-solving with colleagues on participant recruitment strategies. I found myself isolating in my office to think, read, and write, and had to make a conscious effort to discuss my research with others. Moving forward, I know connectedness is essential to building relationships and being a positive leader. In my current role, there are multiple opportunities to connect with students and colleagues regularly, such as committee membership and attending student and faculty gatherings. Moving forward, I will implement what I have learned in the dissertation process, taking time to meet and discuss items with others.

Committee membership. In the beginning of the dissertation, I did not understand the roles and responsibilities of the dissertation committee. I now understand these roles and responsibilities, and appreciate each committee members' input and assistance throughout this journey. As an educational leader, I will not hesitate to form committees and teams to work together to solve problems in addition to assisting students.

In my role as program director for the Master of Science in Nutrition and Dietetics program, I assign research advisors for each graduate student. After seeing the benefits of a research committee, this past semester, instead of assigning one advisor per student, I decided to assign two advisors per student for a couple of the projects. I am coadvisor on both projects. This was done purposefully. One reason was for me to establish strong working relationships and thus enhance connectedness with colleagues. The second reason was for everyone on each research committee to learn from one another regarding research design and identifying problems of practice. Lastly, after 
seeing the benefits of having a committee review and provide feedback on my

dissertation, these research committees will now have a more robust peer-review process.

Problem-solving. The dissertation also influenced my practice as an educational leader by reminding me to draw on the strengths of my colleagues in times of hardship. The outcome of my initial participant recruitment strategy was zero participant volunteers. I then contacted other university faculty and staff to inquire if they would distribute the recruitment flyer to students they had connections to, but received few affirmative responses. The recruitment strategies that worked were recommended by colleagues. Thinking back to that process, I now wonder why I waited so long to ask for guidance from colleagues. I attribute this to my introverted nature and coronavirus disease 2019 (COVID-19), as I chose to isolate in my office, thus having less conversation with others. I learned from the dissertation to nurture relationships, thus increasing connectedness with colleagues, in addition to not hesitating to ask for help from others.

\section{Resilience}

In addition to George et al.'s (2007) authentic leadership characteristics, there are positive psychological attributes which contribute to authentic leadership (Northouse, 2016). These are confidence, hope, optimism, and resilience (Northouse, 2016). Of these four factors, the dissertation process influenced my confidence and resilience, with resiliency aligning more with my practice as an educational leader and confidence aligning more with my practice as a scholar. The areas in which I had to maintain resiliency were regarding COVID-19, IRB approval, and participant recruitment. 
With COVID-19 causing the study site to move to remote learning, I had to pause my research as I needed participants to be on campus to collect data. During those four months, I chose to take a break from research, and focus on work and family. After that break, I emerged refreshed, focused, and determined. I have no doubt there will be other challenges such as this in the future, in which I need to maintain a positive mindset and find how to restructure my thinking, making the best of an unpleasant situation.

Additionally, IRB approval was a learning process where I asked many questions and learned from others who were more experienced than myself in this process. While the process was complicated and took longer than anticipated, I learned a lot and have since mentored others in the process. I must remember to remain resilient in setbacks. I need to learn from those situations, viewing setbacks as learning opportunities not only for myself but also so I am able to help others, so they do not repeat the same mistakes I did. I want to put into practice what I have learned about Nonaka's (1994) modes of knowledge creation and help make my knowledge accessible to others. I am learning to do this through conversation, in addition to identifying and using technology such as Microsoft Teams to share knowledge with colleagues and students.

Participant recruitment was another area I practiced resiliency. After many failed attempts to recruit participants, I used my resources to ask for assistance. This included colleagues, staff, and even the students themselves. The long-term relationships I had built helped me in this process and helped me maintain resiliency. While I had several setbacks in the dissertation process, I now feel more confident in facing challenges as an educational leader. 


\section{Influence on Practice as a Scholar}

The dissertation process influenced me as a scholar by increasing my knowledge, confidence, and resilience in the research process. The increased confidence has led me to initiate creating a faculty research team in addition to beginning to establish my research areas of focus. By creating the research team, I am contributing to building a learning culture (Gill, 2010) around scholarship within the nutrition and dietetics program at a four-year public university.

The nutrition and dietetics program at this university has many strengths, but one weakness is scarcity of scholarly productivity by faculty. This is partly due to lack of mentorship. Now that I am in the final stages of the dissertation process, my knowledge

of the research process and confidence as a researcher have increased significantly. From learning how to identify a relevant problem of practice, to designing the study, and then collecting and analyzing the data, confidence in all areas has increased for me. As both a scholar and educational leader, I decided to take the leap and build a faculty research team with two other colleagues in the nutrition and dietetics program.

A goal of mine since completing oral comprehensive exams with this EdD program, was to begin to establish a learning culture as described by Gill (2010) within the nutrition and dietetics program. Beginning a research team is one step in creating this culture. Gill (2010) states, "well-functioning teams have members who learn together and learn how to learn together" (p. 76). Although all three of the members in this research team are novice researchers, we openly shared our research strengths and weaknesses from the beginning. We then assisted one another throughout the research process based upon that knowledge. In our initial meeting, we individually shared our 
vision, team goals, and personal goals for the research team as encouraged by Gill (2010) and Levi (2017), and then came to an agreement on our team vision and goals for the academic year. We also discussed group norms such as encouraging all team members to openly share their thoughts (Levi, 2017). Additionally, roles and responsibilities for each task were discussed and delegated according to one another's strengths and personal goals.

This contributed to creating trust within our team and thus led to building a safe and open environment for all team members to share ideas (Levi, 2017). Within four months, the three of us wrote and submitted a grant, applied to present at a national conference, and completed an IRB application to begin a research study in February 2021. Without having gone through the dissertation process, this research team would not exist. I now have the knowledge and confidence to continue working on research projects and look forward to future scholarly endeavors.

I also learned from the dissertation you cannot rush the writing process and instead need to be patient and enjoy the process. There were times where I had writer's block, and wondered where and how the words would come. I learned to stop, take a walk, and think, or move to a different task and then come back to the dissertation on a different day. As we learned from this EdD program, you need to know four primary items to complete a dissertation. These are knowing how to think, read, write, and talk. Many times, I needed to spend more time thinking and reading to overcome the writer's block. Other times I needed to discuss my thoughts with others. This I will carry with me as I continue to strengthen my research skills and begin to make contributions to scholarship as an assistant professor. 
Lastly, the dissertation had an impact on how I value scholarship in addition to how I contribute to conversations regarding scholarship. After reading a plethora of articles about my research topic and having experienced the amount of focus and determination required to see a research project through to the end, I have a deep appreciation for scholars and scholarly work. Additionally, I am now motivated to contribute to this work and am starting to internalize the identity of scholar. Finally, the dissertation increased my confidence in research. I am beginning to speak like a researcher and contribute to conversation about research with colleagues and students. Recently, I volunteered to co-chair an interprofessional education writing group. I hope to continue to expand my network of researchers, finding ways to collaborate with researchers across disciplines.

\section{Conclusion}

The dissertation process has positively influenced my practice as an educational leader and as a scholar. While my research knowledge and skills have increased, so have the leadership attributes of resilience, connection, and team building. I appreciate all I have learned from this EdD program, including the dissertation process, and look forward to continuing my research journey. 


\section{References}

Aimé, A., Villatte, A., Cyr, C., \& Marcotte, D. (2017). Can weight predict academic performance in college students? An analysis of college women's self-efficacy, absenteeism, and depressive symptoms as mediators. Journal of American College Health, 65(3), 168-176. doi:10.1080/07448481.2016.1266639

American Association of University Professors, (AAUP). (1966). Statement on government of colleges and universities. Retrieved from https://www.aaup.org/report/statement-government-colleges-and-universities

American College Health Association. (2020). National College Health Assessment III: Reference Group Executive Summary Spring 2020. Silver Spring, MD: American College Health Association.

American Psychological Association. (2021). Stigma and Health. Retrieved from https://www.apa.org/pubs/journals/sah?tab=4

Andreyeva, T., Puhl, R. M., \& Brownell, K. D. (2008). Changes in perceived weight discrimination among Americans, 1995-1996 through 2004-2006. Obesity, 16, 1129-1134. doi:10.1038/oby.2008.35

Association for Size Diversity and Health, (ASDAH). (2018). HAES principles. Retrieved from https://www.sizediversityandhealth.org/content.asp?id=76

Bacon, L., \& Aphramor, L. (2011). Weight science: Evaluating the evidence for a paradigm shift. Nutrition Journal, 10(9) 1-13. doi:10.1186/1475-2891-10-9

Bardach, E., \& Patashnik, E. M. (2016). A practical guide for policy analysis: The eightfold path to more effective problem solving (5th ed.). Los Angeles, CA: Sage.

Bates, E. A., McCann, J. J., Kaye, L. K., \& Taylor, J. C. (2017). "Beyond words": A 
researcher's guide to using photo elicitation in psychology. Qualitative Research in Psychology, 14(4), 459-481.

Benson, R., von Hippel, P. T., \& Lynch, J. L. (2017). Does more education cause lower BMI, or do lower-BMI individuals become more educated? Evidence from the National Longitudinal Survey of Youth 1979. Social Science \& Medicine, 211, 370-377.

Berg, F. M. (1999). Integrated approach: Health at any size. Healthy Weight Journal, 13(5), 74.

Burmeister, J. M., Taylor, M. B., Rossi, J., Kiefner-Burmeister, A., Borushok, J., \& Carels, R. A. (2016). Reducing obesity stigma via a brief documentary film: A randomized trial. Stigma and Health, 2(1), 43-52.

Cameron, E. (2016). Challenging "Size Matters" messages: An exploration of the experiences of critical obesity scholars in higher education. Canadian Journal of Higher Education, 46(2), 111-126.

Cervero, R. M., \& Wilson, A. L. (2006). Working the planning table: Negotiating democratically for adult, continuing, and workplace education. San Francisco, CA: Jossey-Bass.

Chang, V. W. \& Christakis, N. A. (2003). Self-perception of weight appropriateness in the United States. American Journal of Preventive Medicine, 24(4), 332-339. doi: 10.1016/S0749-3797(03)00020-5

Clif's Notes. (2019, October 15). The success in our international enrollment is a model for what we must do at MSU [Web log post]. Retrieved from https://blogs.missouristate.edu/president/2019/10/15/clifs-notes-for-oct-15-2019/ 
Collier, J., Jr., \& Collier, M. (1986). Visual anthropology: Photography as a research method (3rd ed.). Albuquerque, NM: University of New Mexico Press.

Crandall, C. S. (1995). Do parents discriminate against their heavyweight daughters? Personality and Social Psychology Bulletin, 21, 724-735. doi:10.1177/0146167295217007

Creswell, J. W. (2013). Qualitative inquiry \& research design: Choosing among five approaches (3rd ed.). Los Angeles, CA: Sage.

Creswell, J. W. (2014). Research design: Qualitative, quantitative, and mixed method approaches (4th ed.). Los Angeles, CA: Sage.

Creswell, J. W. (2016). 30 essential skills for the qualitative researcher. Los Angeles, CA: Sage.

Crosnoe, R. (2007). Gender, obesity, and education. Sociology of Education, 80(3), 241260.

Dutton, G. R., Lewis, T. T., Durant, N., Halanych, J., Kiefe, C. I., Sidney, S., Kim, Y., \& Lewis, C. E. (2014). Perceived weight discrimination in the CARDIA study: Differences by race, sex, and weight status. Obesity, 22(2), 530-536.

Elo, S., \& Kyngäs, H. (2008). The qualitative content analysis process. Journal of Advanced Nursing, 62(1), 107-115. doi:10.1111/j.1365-2648.2007.04569.x

Fowler-Brown, A. G., Ngo, L. H., Phillips, R. S., \& Wee, C. C. (2010). Adolescent obesity and future college degree attainment. Obesity, 18(6), 1235-1241.

George, B., Sims, P., Mclean, A. N., \& Mayer, D. (2007). Discovering your authentic leadership. Harvard Business Review, 85(2), 129-138.

Gill, S. J. (2010). Developing a learning culture in nonprofit organizations. Thousand 
Oaks, CA: Sage.

Gloor, J. L., \& Puhl, R. M. (2016). Empathy and perspective-taking: Examination and comparison of strategies to reduce weight stigma. Stigma and Health, 1(4), 269279. doi: $10.1037 / \mathrm{sah} 0000030$

Gray, D. E. (2018). Doing research in the real world (4th ed.). Los Angeles, CA: Sage.

Han, L., Duan, S., Qi, L. You, D., Zeng, F., Feng, X., \& Astell-Burt, T. (2019). Trends in self-perceived weight status, weight loss attempts, and weight loss strategies among adults in the United States, 1999-2016. JAMA Network Open, 2(11), e1915219. doi: 10.1001/jamanetworkopen.2019.15219

Harper, D. (2002). Talking about pictures: A case for photo elicitation. Visual Studies, 17(1), 13-26. doi:10.1080/14725860220137345

Hatzenbuehler, M. L., Keyes, K. M., \& Hasin, D. S. (2009). Associations between perceived weight discrimination and the prevalence of psychiatric disorders in the general population. Obesity, 17(11), 2033-2039.

Heifetz, R. A. (1994). Leadership without easy answers. Cambridge, MA: Harvard University Press.

Heuer, C. A., McClure, K. J., \& Puhl, R. M. (2011). Obesity stigma in online news: A visual content analysis. Journal of Health Communication, 16, 976-987.

Hsieh, H. F., \& Shannon, S. E. (2005). Three approaches to qualitative content analysis. Qualitative Health Research, 15(9), 1277-1288.

Humphrey, L., Clifford, D., \& Neyman Morris, M. (2015). Health at Every Size college course reduces dieting behaviors and improves intuitive eating, body esteem, and anti-gay attitudes. Journal of Nutrition Education and Behavior, 47(4), 354-360. 
Incollingo Rodriguez, A. C., White, M. L, Standen, E. C., Mann, T., Wells, C. R., \& Tomiyama, A.J. (2019). Body mass index and educational inequality: An update of Crandall (1995). Stigma and Health, 4(3), 357-363.

Ingram, D. D., \& Mussolino, M. E. (2010). Weight loss from maximum body weight and mortality: The Third National Health and Nutrition Examination Survey Linked Mortality File. International Journal of Obesity, 34(6), 1044-1050.

Janssen, I., \& Mark, A. E. (2006). Elevated body mass index and mortality risk in the elderly. Obesity Reviews, 8, 41-59.

Krueger, R. A., \& Casey, M. A. (2015). Focus groups: A practical guide for applied research (5th ed.). Los Angeles, CA: Sage.

Kuckartz, U. (2014). Qualitative text analysis: A guide to methods, practice \& using software. Los Angeles, CA: Sage.

Kylstra, C. (2018, June 25). How should a health brand talk about weight? An evolving style guide. SELF. Retrieved from: https://www.self.com/story/self-weighthealth-style-guide

Lancet Public Health Editorial. (2019, April). Addressing weight stigma. The Lancet, 4, e168.

Levi, D. (2017). Group dynamics for teams (5th ed.). Los Angeles, CA: Sage.

Loss, C. P., \& McGuinn, P. J. (2016). The convergence of K-12 and higher education: Policies and programs in a changing era. Cambridge, MA: Harvard Education Press.

Manning, K. (2013). Organizational theory in higher education. New York, NY: Routledge. 
McVey G. L., Gusella J., Tweed S., \& Ferrari M. (2009). A controlled evaluation of Web-based training for teachers and public health practitioners on the prevention of eating disorders. Eating Disorders, 17(1), 1-26.

McVey, G. L., Walker, K. S., Beyers, J., Harrison, H. L., Simkins, S. W., \& RussellMayhew, S. (2013). Integrating weight bias awareness and mental health promotion into obesity prevention delivery: A public health pilot study. Preventing Chronic Disease, 10, 1-7. doi:10.5888/pcd10.120185

Mensinger, J. L., Tylka, T. L., \& Calamari, M. E. (2018). Mechanisms underlying weight status and healthcare avoidance in women: A study of weight stigma, bodyrelated shame and guilt, and healthcare stress. Body Image, 25, 139147. doi:10.1016/j.bodyim.2018.03.001

Merriam, S. B., \& Tisdell, E. J. (2016). Qualitative research: A guide to design and implementation (4th ed.). San Francisco, CA: Jossey-Bass.

Merriam-Webster Dictionary. (2020). Bias. Retrieved from https://www.merriamwebster.com/dictionary/bias

Michigan Department of Civil Rights. (2019). Civil rights guaranteed by law. Retrieved from https://www.michigan.gov/mdcr/0,4613,7-138-4954_4997-16288--,00.html

Miller, W. C., \& Jacob, A. V. (2001). The health at any size paradigm for obesity treatment: The scientific evidence. Obesity Reviews, 2(1), 37-45.

Mintzberg, H. (2005). The five basic parts of the organization. In J. M. Shafritz, J. S. Ott, \& Y. S. Jang (Eds.), Classics of organization theory (6th ed., pp. 219-230). Belmont, CA: Wadsworth. (Reprinted from The structure of organization: A synthesis of research, pp. 18-34, 1979, Upper Saddle River, NJ: Prentice Hall) 
Missouri State Office of Institutional Research. (2020a). Bear Stats. Retrieved from https://www.missouristate.edu/oir/Bear-Stats.htm

Missouri State Office of Institutional Research. (2020b). Bear Fact Book:

Springfield campus 2018-2019. Retrieved from

https://www.missouristate.edu/Assets/oirfactbook/Factbook-FINAL-18-19.pdf

Missouri State University. (2016a). 2016-21 long-range plan: Diversity and inclusion.

Retrived from https://www.missouristate.edu/longrangeplan/diversityinclusion.htm

Missouri State University. (2016b.) Non-discrimination policy statement. Retrieved from https://www.missouristate.edu/policy/G1_05_NonDiscriminationPolicy.htm

Missouri State University. (2018a). What is public affairs. Retrieved from https://publicaffairs.missouristate.edu/About.htm

Missouri State University. (2018b). About Missouri State mission, vision and values. Retrieved from https://www.missouristate.edu/about/missionstatement.htm

Missouri State University. (2019a). About Missouri State. Retrieved from https://www.missouristate.edu/about/

Missouri State University. (2019b). Policy Library. Retrieved from https://www.missouristate.edu/policy/

Missouri State University. (2019c). Key performance indicators. Retrieved from https://mis.missouristate.edu/KeyPerformanceIndicators/kpi2016/index Missouri State University. (2019d). First-generation student support. Retrieved from https://www.missouristate.edu/firstgeneration/ 
Missouri State University. (2019e). Diversity and inclusion shattering the silence series.

Retrieved from https://diversity.missouristate.edu/shattering-the-silence.htm Missouri State University. (2020). Office for insitutional equity and compliance.

Retrieved from https://www.missouristate.edu/equity/

Missouri State University. (2021a). Diversity and inclusion vision and mission. Retrieved from https://diversity.missouristate.edu/Vision.htm

Missouri State University. (2021b). Diversity and inclusion implementation strategy. Retrieved from https://diversity.missouristate.edu/diversity-and-inclusion.htm Missouri State University Board of Governors. (2019, Oct.). Strategic Enrollment Management Update. Retrieved from https://www.missouristate.edu/Assets/enrollmentmanagement/BOG-SEMUpdate-2019-10-25.pdf

Missouri State University Faculty Senate Agenda. (2017, Mar.). Policy library report. Retrieved from https://www.missouristate.edu/assets/facultysenate/ FS_March_2017_Agenda_Attachments_v2.pdf

Nonaka, I. (1994). A dynamic theory of organizational knowledge creation. Organization Science, 5(1), 14-37.

Northouse, P. G. (2016). Leadership: Theory and practice (7th ed.). Los Angeles, CA: Sage.

Nutter, S., Russel-Mayhew, S., Alberga, A. S., Arthur, N., Kassen, A., Lund, D. E., Sesma-Vazquez, M., \& Williams, E. (2016). Positioning of weight bias: Moving towards a social justice issue. Journal of Obesity, 2016, 1-10. doi:10.1155/2016/3753650 
Odlaug, B. L., Lust, K., Wimmelmann, C. L., Chamberlain, S. R., Mortensen, E. L., Derbyshire, K., Christenson, G., \& Grant, J. E. (2015). Prevalence and correlates of being overweight or obese in college. Psychiatry Research, 227(1), 58-64.

O’Hara, L., \& Gregg, J. (2006). The war on obesity: A social determinant of health. Health Promotion Journal of Australia, 17(3), 260-263.

O’Hara, L., Tahboub-Schulte, S., \& Thomas, J. (2016). Weight-related teasing and internalized weight stigma predict abnormal eating attitudes and behaviours in Emirati female university students. Appetite, 102, 44-50. doi:10.1016/j.appet.2016.01.019

O’Hara, L., \& Taylor, J. (2018). What's wrong with the 'war on obesity?' A narrative review of the weight-centered health paradigm and development of the $3 \mathrm{C}$ framework to build critical competency for a paradigm shift. SAGE Open, 8(2), 128.

O’Reilly, C., \& Sixsmith, J. (2012). From theory to policy: Reducing harms associated with the weight-centered health paradigm. Fat Studies, 1(1), 97-113.

Pearl, R. L., Dovidio, J. F., \& Puhl, R. M. (2015). Visual portrayals of obesity in health media: Promoting exercise without perpetuating weight bias. Health Education Research, 30(4), 580-590.

Pearl, R. L., Puhl, R. M., \& Dovidio, J. F. (2017). Can legislation prohibiting weight discrimination improve psychological well-being? A preliminary investigation. Analyses of Social Issue and Public Policy, 17(1), 84-104.

Pescosolido, B. A., \& Martin, J. K. (2015). The stigma complex. Annual Review of Sociology, 41, 87-116. 
Puhl, R. (2019, June 21). Perspective | Weight discrimination is rampant. Yet in most places it's still legal. Washington Post. Retrieved from https://www.washingtonpost.com/outlook/weight-discrimination-isrampant-yet-in-most-places-its-still-legal/2019/06/21/f958613e-9394-11e9-b72dd56510fa753e_story.html

Puhl, R. M., \& Brownell, K. D. (2001). Bias, discrimination, and obesity. Obesity Research, 9(12), 788-805. doi:10.1038/oby.2001.108

Puhl, R. M., \& Brownell, K. D. (2006). Confronting and coping with weight stigma: An investigation of overweight and obese adults. Obesity, 14(10), 1802-1815.

Puhl, R. M., \& Heuer, C. A. (2009). The stigma of obesity: A review and update. Obesity, 17(5), 941-964.

Puhl, R. M., \& Heuer, C. A. (2010). Obesity stigma: Important considerations for public health. American Journal of Public Health, 100, 1019-1028. doi:10.2105/AJPH.2009.159491

Puhl, R. M., \& King, K. M. (2013). Weight discrimination and bullying. Best Practice \& Research Clinical Endocrinology \& Metabolism, 27, 117-127.

Puhl, R. M., Andreyeva, T., \& Brownell, K. D. (2008). Perceptions of weight discrimination: Prevalence and comparison to race and gender discrimination in America. International Journal of Obesity, 32, 992-1000.

Puhl, R. M., Himmelstein, M. S. \& Quinn, D. M. (2018). Internalzing weight stigma: Prevalence and sociodemographic considerations in US adults. Obesity, 26(1), $167-75$.

Puhl, R. M., Luedicke, J., \& Heuer, C. A. (2013). The stigmatizing effect of visual media 
portrayals of obese persons on public attitudes: Does race or gender matter? Journal of Health Communication, 18, 805-826.

Puhl, R., Luedicke, J., \& Peterson, J. L. (2013). Public reactions to obesity-related health campaigns: A randomized controlled trial. American Journal of Preventive Medicine, 45(1), 36-48

Puhl, R. M., Moss-Racusin, C. A., Schwartz, M. B., \& Brownell, K. D. (2008). Weight stigmatization and bias reduction: perspectives of overweight and obese adults. Health Education Research, 23(2), 347-358.

Puhl, R., Peterson, J. L., \& Luedicke, J. (2013). Fighting obesity or obese persons? Public perceptions of obesity-related health messages. International Journal of Obesity, 37(6), 774-782.

Puhl, R. M., Suh, Y., \& Li, X. (2016). Legislating for weight-based equality: National trends in public support for laws to prohibit weight discrimination. International Journal of Obesity, 40(8), 1320-1324.

Pursey, K., Burrows, T. L, Stanwell, P., \& Collins, C. E. (2014). How accurate is webbased self-reported height, weight, and body mass index in young adults? Journal of Medical Internet Research, 16(1), 1-9. doi: 10.2196/jmir.2909

Ramos-Salas, X., Alberga, A. S., Cameron, E., Estey, L., Forhan, M., Kirk, S. F. L., Russell-Mayhew, S., \& Sharma, A. M. (2017). Addressing weight bias and discrimination: Moving beyond raising awarenss to creating change. Obesity Reviews, 18, 1323-1335.

Robison, J. (1997). Weight management: Shifting the paradigm. Journal of Health Education, 28(1), 28-34. 
Rote, A. E., Love, S. P., Lakatos, C. M., \& Hewitt, L. (2018). Development and assessment of a course to reduce weight bias in undergraduate health promotion students. Pedagogy in Health Promotion, 4(3), 200-210. doi:10.1177/2373379917742925

Ryan, F., Coughlan, M. \& Cronin, P. (2007). Step-by-step guide to critiquing research. Part 2: Qualitative research. British Journal of Nursing, 16(12), 738-744.

S. Res. 7, Missouri State University Faculty Senate. 7 (2017, Mar. 9). Retrieved from https://www.missouristate.edu/assets/facultysenate/FS_Minutes_March_2017_c omplete_session_-_approved.pdf

Stevens, C. (2018). Fat on campus: Fat college students and hyper(in)visible stigma. Sociological Focus, 51(2), 130-149. doi:10.1080/00380237.2017.1368839

Sutin, A. R., \& Terracciano, A. (2017). Sources of weight discrimination and health. Stigma and Health, 2(1), 23-27. doi:10.1037/sah0000037

Thorel, N., Thorel, E., Tuschen-Caffier, B. (2020). Differential stigmatization in the context of eating disorders: Less blame might come at the price of greater social rejection. Stigma and Health. Advance online publication. doi:10.1037/sah0000274.

Tracy, S. J. (2010). Qualitative quality: Eight "big-tent" criteria for excellent qualitative research. Qualitative Inquiry, 16(10), 837-851. doi: 10.1177/1077800410383121

Trainer, S., Brewis, A., Williams, D., \& Rosales-Chavez, J. (2015). Obese, fat, or “just big”? Young adult deployment of and reactions to weight terms. Human Organization, 74(3), 266-275.

Tylka, T. L., Annunziato, R. A., Burgard, D., Daníelsdóttir, S., Shuman, E., Davis, C., \& 
Calogero, R. M. (2014). The weight-inclusive versus weight-normative approach to health: Evaluating the evidence for prioritizing well-being over weight loss. Journal of Obesity, 2014, 1-18. doi:10.1155/2014/983495

Vartanian, L. R., Pinkus, R. T., \& Smyth, J. M. (2014). The phenomenology of weight stigma in everyday life. Journal of Contextual Behavioral Science, 3(3), 196-202. doi:10.1016/j.jcbs.2014.01.003

Webb, J. B., Fiery, M. F., \& Jafari, N. (2016). "You better not leave me shaming!": Conditional indirect effect analyses of anti-fat attitudes, body shame, and fat talk as a function of self-compassion in college women. Body Image, 18, 5-13.

Wellman, J. D., Araiza, A. M., Newell, E. E., \& McCoy, S. K. (2018). Weight stigma facilitates unhealthy eating and weight gain via fear of fat. Stigma and Health, 3(3), 186-194. doi:10.1037/sah0000088

Yin, R. K. (2014). Case study research: Design and methods (5th ed.). Los Angeles, CA: Sage.

Young, R., Subramanian, R., \& Hinnant, A. (2016). Stigmatizing images in obesity health campaign messages and healthy behavioral intentions. Health Education \& Behavior, 43(4), 412-419. 
Appendix A

Recruitment Post in Student Wellness Newsletter

VOLUNTEERS NEEDED FOR RESEARCH STUDY ON

WEIGHT STIGMA

\section{What is the research about?}

This research study is about exploring college student perspectives of messaging at Missouri State, and whether the messages use weight inclusive content, or weight stigmatizing content.

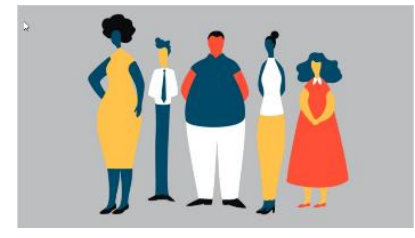

I hope to increase awareness of weight stigma and contribute knowledge on how higher education institutions can promote weight inclusive environments to aid in decreasing weight stigma for all students.

\section{What is involved?}

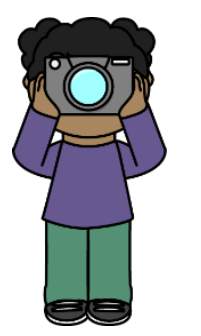

- Complete a 2-minute demographic survey

- Meet with researcher for a 30-minute virtual meeting via Zoom to discuss your role in taking photographs

- Using your own device, take five or more photos over a two-week period, capturing examples of weight inclusive and weight stigmatizing university messaging and email photos to the researcher

- Complete a brief online questionnaire about each photo

Total time requirement for a participant in this study is estimated to be $\mathbf{1} \mathbf{~ h r} \mathbf{2 2} \mathbf{~ m i n}$.

\section{Are you eligible to participate?}

Eligible participants need to self-identify as a person of larger size, and be 18 years of age or older

\section{Want to get involved and help decrease weight stigma?}

If you would like to participate in this study or have questions, please contact me at HRoberts@missouristate.edu. Or, if you prefer you may go ahead and access the consent form, to decide whether or not you would like to participate in the study. You can find it hyperlinked above or click here: https://missouristate.col.qualtrics.com/jfe/form/SV_5w1DAYqykfKxCCN

If you choose to participate, you will click the "I consent, begin the study" button on the form, and then a demographic survey will begin. If you choose not to participate, you will click the "I do not consent, I do not wish to participate" button and the session will end. 
Thank you for considering being a part of this study.

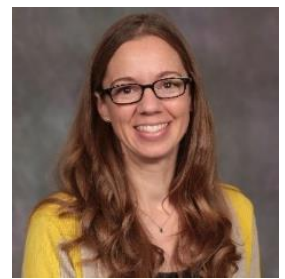

Hillary Roberts

Doctoral student in the Educational Leadership and Policy Analysis program with the University of Missouri-Columbia and Missouri State alumna 
Appendix B

Research Participation Recruitment Email and Flyer

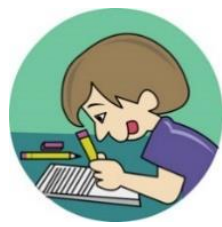

Hello Missouri State Student,

My name is Hillary Roberts, and I am a doctoral student in the Educational Leadership and Policy Analysis program with the University of MissouriColumbia. You can learn about this study by reading below or viewing this video.

\section{What is the research about?}

My research study is about exploring college student perspectives of messaging at Missouri State, and whether the messages use weight inclusive content, or weight stigmatizing content.

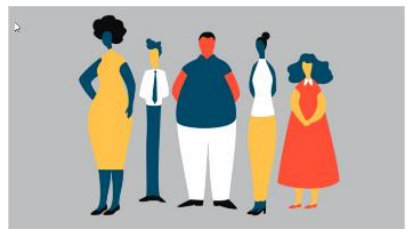

I hope to increase awareness of weight stigma and contribute knowledge on how higher education institutions can promote weight inclusive environments to aid in decreasing weight stigma for all students.

\section{What is involved?}

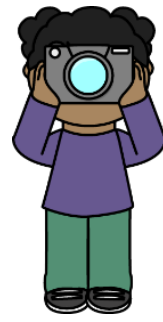

- Complete a 2-minute demographic survey

- Meet with researcher for a 30-minute virtual meeting via Zoom to discuss your role in taking photographs

- Using your own device, take five or more photographs over a two-week period, capturing examples of weight inclusive and weight stigmatizing university messaging and email photos to the researcher

- Complete a brief online questionnaire about each photo

The total time requirement for a participant in this study is estimated to be 1 hour 22 minutes. This includes the 30-minute virtual meeting with the researcher.

\section{Are you eligible to participate?}

- Eligible participants need to self-identify as a person who is of larger size, and be 18 years of age or older

\section{Want to get involved and help decrease weight stigma?}

If you would like to participate in this study or have questions, please contact me at HRoberts@missouristate.edu or 417-836-8884. Or, if you prefer you may go ahead and access the consent form, to decide whether or not you would like to participate in the study. You can find it hyperlinked above or click here: https://missouristate.col.qualtrics.com/jfelform/SV_5w1DAYqykfKxCCN

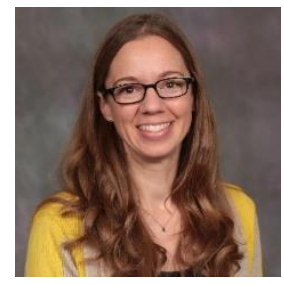

Thank you for considering being a part of this study. -Hillary 
Appendix C

Informed Consent Form

\section{Title of Project: A Case Study of College Students and Weight Inclusive Messages: Implications for University Non-Discrimination Policy Investigator: Hillary Roberts, MS, RDN}

Introduction: You have been asked to participate in a research study. Before you agree to participate in this study, it is important that you read and understand the following explanation of the study and the procedures involved. Please take as much time as you need to read this consent form.

If you have any questions about the study or your role in it at any time, be sure to call or email the investigator. You may contact the primary investigator at:

Hillary Roberts

hroberts@missouristate.edu

$417-836-8884$

You will need to consent to this study giving your permission to be involved in the study by clicking "I consent, begin the study" found at the bottom of this page. Taking part in this study is entirely your choice. If you decide to take part but later change your mind, you may stop at any time. If you decide to stop, you do not have to give a reason, and there will be no negative consequences for ending your participation.

Purpose of this Study: The purpose of this study is to explore college student perspectives of messaging at Missouri State University, and whether messages are perceived as weight inclusive or weight stigmatizing. To do this, you have been asked to participate in photo-elicitation where you will take photos of messaging you see at Missouri State. It is expected that 30 people will participate in this study.

Your Participation: If you agree to be part of this study, you will be asked take part in a four-step process:

1. Complete demographic survey. Estimated time to complete this survey is two (2) minutes.

2. Meet with researcher for a 30-minute virtual meeting via Zoom to discuss the participant's role in the study including the photo-elicitation instructions.

3. Using your own device, take five or more photos over a two-week period at Missouri State University. Then email each photo to the researcher.

4. Complete an online questionnaire for each photo taken. Taking a photo, emailing it to the researcher, and completing the questionnaire about each photo is estimated to take ten (10) minutes to complete for each photo.

Time Requirements: Photo-elicitation will occur for two weeks. Taking a photo, emailing it to the researcher, and completing the questionnaire about each photo is estimated to take ten (10) minutes to complete for each photo. 
If you take five photos, the minimum number of photos requested, estimated total time is 50 minutes for the photos plus 2 minutes to take the demographic survey and 30 minutes for the photo-elicitation instruction meeting, for a total time requirement of 1 hour 22 minutes.

Participation risks: There are no known risks to you as a result of participating in this study.

Participation benefits: You may not directly benefit from this study. However, information from this study will hopefully increase awareness of weight stigma and contribute knowledge on how the messaging and policies of higher education institutions can be used to promote weight inclusive environments to aid in decreasing weight stigma for all students.

Confidentiality: The results of this study will be confidential and only the investigator will have access to the information. All digital data for this research will be stored in the researcher's electronic/computer files. Computer files are protected with a password and the computer is in a locked office that only the researcher can open.

Privacy: At no time will your actual identity be revealed. Your name or personal identifying information will not be used in any published reports of this research.

We will do our best to make sure that your personal information from this study is kept private, but we cannot guarantee total privacy. We may give out your personal information if the law requires it. If we publish the results of this study or present them at scientific meetings, we will not use your name or other personal information.

We will keep the information we collect from you for this study to use in future research/to share with other investigators to use in future studies without asking for your consent again. Information that could identify you will be removed from your research information so no one will know that it belongs to you.

Additionally, you must give us permission to use the photographs you take during the study. Clicking the box, "I consent, begin the study," on this consent form gives the researchers permission to use the photographs you take.

Voluntary participation and withdrawal: Your participation in this study is voluntary, and you may decline to participate or withdrawal from the study at any time. Your decision to participate or not to participate will have no effect on your course grades. You may withdraw by informing the researcher that you no longer wish to participate.

The investigator will keep the information collected from you for this study to use in future research/to share with other investigators to use in future studies without asking for your consent again. Information that could identify you will be removed from your research information so no one will know that it belongs to you.

Compensation: You will not be paid or given extra credit for taking part in this study. 
Who to call with questions, concerns, or complaints: If you have more questions about this study at any time, you can contact Hillary Roberts at 417-836-8884, hlcrtz@mail.missouri.edu, or Dr. Cynthia MacGregor at 417-836-6046, macgregorcj@missouri.edu.

You may contact the University of Missouri Institutional Review Board (IRB) if you: Have any questions about your rights as a study participant; Want to report any problems or complaints; or Feel under any pressure to take part or stay in this study. The IRB is a group of people who review research studies to make sure the rights of participants are protected. Their phone number is 573-882-3181.

If you want to talk privately about your rights or any issues related to your participation in this study, you can contact University of Missouri Research Participant Advocacy by calling 888-280-5002 (a free call) or emailing MUResearchRPA@missouri.edu.

If you choose to participate in the study, the researcher will email you a copy of this consent form.

\section{Consent to Participate:}

By clicking the button below, you acknowledge:

- You have read and understand the information in this form.

- Your participation in the study is voluntary.

- You are 18 years of age.

- You are aware that you may choose to terminate your participation at any time for any reason.

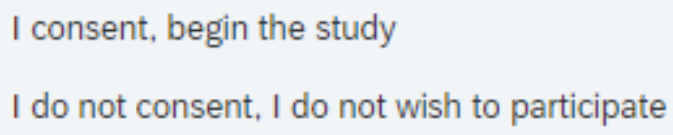




\section{Appendix D}

\section{Screening Email Script}

Hello (name of student),

Thank you for your interest in being a part of this study, A Case Study of College Students and Weight Inclusive Messages: Implications for University Non-Discrimination Policy.

The next step is reading the consent form and deciding whether or not you would like to participate in the study. You can find it hyperlinked above or click here: https://missouristate.col.qualtrics.com/jfe/form/SV_5w1DAYqykfKxCCN

If you choose to participate, you will click the "I consent, begin the study" button on the form, and then a demographic survey will begin. If you choose not to participate, you will click the "I do not consent, I do not wish to participate" button and the session will end.

The demographic survey is expected to take 2-minutes to complete. You will be asked to type what body size you identify as in addition to your age in years. This is because to be eligible to participate in this study you need to self-identify as a person who is overweight or has obesity and be 18 years of age or older. If you self-identify as a person who is overweight or has obesity, I will then email you to set-up a 30-minute Zoom virtual meeting to discuss the process for taking pictures of messages found on campus in addition to answering any questions you have about the consent form or the study in general.

The total time commitment for your participation is estimated to be around 1 hour 22 minutes.

It is my hope this study will increase awareness of weight stigma and contribute knowledge on how the messaging and policies of higher education institutions can be used to promote weight inclusive environments to aid in decreasing weight stigma for all students.

Thank you for your consideration in being a part of this research.

Hillary

Hillary Roberts

Doctoral Student

HRoberts@missouristate.edu

417-836-8884 
Appendix E

\section{Sociodemographic Survey}

Thank you for your interest in being a participant in the research study, A Case Study of College Students and Weight Inclusive Messages: Implications for University Non-

Discrimination Policy.

Please complete this survey.

Only the researcher can view your results.

If you meet the criterion to be a participant in this study, the researcher will contact you via your university email address within the next 7-days.

What is your gender?

- Female

- Male

- Other (specify)

What is your age in years?

Please select which body size or weight category with which you identify.

- Underweight (survey ends if participant selects this option)

- Normal weight (survey ends if participant selects this option)

- Healthy weight (survey ends if participant selects this option)

- Overweight

- Obese

- Other (specify):

If you are comfortable, please explain what leads you to identify as this body size or weight category?

Which academic college, if any, is your declared major within?

- Darr College of Agriculture (e.g., Agriculture, Animal Science, Pre-Veterinary)

- College of Arts and Letters (e.g., English, Communication, Music)

- College of Business (e.g., Information Technology and Cybersecurity, Management, Merchandising and Fashion Design)

- College of Education (e.g., Child and Family Development, Family and Consumer Science, Early Childhood Education)

- McQueary College of Health and Human Services (e.g., Cell and Molecular Biology, Speech Language Pathology, Exercise and Movement Science, Psychology)

- College of Humanities and Public Affairs (e.g., Criminology, Economics, Religious Studies) 
- College of Natural and Applied Sciences (e.g., Biology, Chemistry, Hospitality Leadership, Computer Science)

- None; undeclared major

Lastly, with which race do you identify?

- White

- Black or African American

- Hispanic

- Asian

- Latino/a

- American Indian or Alaska Native

- Other (specify)

Please type your university email address so the researcher can contact you within the next 7-days to discuss the next step in this study of taking photos on campus.

Please leave any additional information that you would like us to know if the space provided below. Thank you! 


\section{Appendix F}

\section{Photo-Elicitation Instructions}

Photo-elicitation is a student-centered approach to gain student insights on their experiences.

This research study will explore your perspectives of messaging at Missouri State University, and whether the messages use weight inclusive written and visual content, or weight stigmatizing written and visual content.

It is the researcher's hope that you are able to take photos that capture the essence of your experiences regarding weight inclusive and weight stigmatizing messages.

\section{What is involved?}

If you would like to participate in this study, you will be asked to:

- Take five or more photographs over a two-week period, capturing examples of weight inclusive and weight stigmatizing university messaging

- Email photos to the researcher using your Missouri State live accounts

- Complete a brief online questionnaire about each photo. This is estimated to take around four to six minutes per photo.

When: For a two-week period from today to Oct 31, 2020.

Where: Anywhere on Missouri State University campus (i.e., residence hall, classroom, recreation center, PSU, etc.). The dining centers on excluded due to the messaging there being from a contracted agency.

What: Any type of MSU messaging. You are asked to take five or more photographs that capture examples of weight inclusive and weight stigmatizing university messaging. Messaging includes visual images and written content (i.e., flyer, banner, brochure, student organization items).

\section{Use smartphone to take photo and then email photo to:}

- Hillary Roberts at HRoberts@ missouristate.edu

- In Subject line type: (a) picture, (b) photo, or (c) image

You will then receive a link via email to complete a brief survey on the photo. Survey questions are below.

1. What experiences (feelings or thoughts) does this photo elicit in you? Please explain your answer.

2. Do you find the message to be weight inclusive or weight stigmatizing? Please explain:

3. Why did you choose to take this photo?

4. Where is the message located that you photographed? (For example, residence hall bulletin board, Meyer Library, Foster Recreation Center)

5. Please type your initials so I know what photo you are talking about here. Thank you.

All emailed photographs will be transferred to a secure and confidential SharePoint site. 


\section{Appendix G}

\section{Photo-Elicitation Questionnaire}

1. What experiences (feelings or thoughts) does this photo elicit in you?

Please explain your answer.

2. Do you find the message to be weight inclusive or weight stigmatizing?

Please explain.

3. Why did you choose to take this photo?

4. Where is the message located that you photographed? (For example, banner outside of Carrington, residence hall bulletin board, recreation center) 


\section{Appendix $\mathrm{H}$}

\section{Photo-Elicitation Questionnaire Email}

Your photo has been received.

The next step is to complete the Photo-Elicitation Questionnaire.

Thank you for the photo and your responses on the questionnaire.

Your experiences of weight stigma and weight inclusiveness that you are sharing through pictures and the thoughtful responses on the questionnaire are much appreciated.

Thank you,

Hillary

\section{Hillary Roberts}

EdD Student

University of Missouri-Columbia 


\section{Appendix I}

\section{Document Policy Analysis Protocol}

\section{Weight Inclusive Language}

1. The policy recommends a behavior that would be encouraged to people of all sizes.

2. The policy uses person-first language (e.g., person with obesity instead of obese person).

3. The policy uses health-focused terms.

4. The policy promotes flexible, individualized eating based on internal cues of hunger, satiety, pleasure, appetite, and individual nutrition needs.

5. The policy does not assign moral values to food choices, instead using objective terms (e.g., healthy food, unhealthy food).

6. The policy promotes life-enhancing physical activity that allows people of all sizes, ability, and interests to engage in enjoyable movement, to the degree they choose.

7. The policy decouples health and weight, not using body mass index (BMI) as an indicator of health status.

\section{Weight Stigmatizing Language}

1. The policy recommends a behavior that would be encouraged only to people who are overweight or have obesity.

2. The policy does not use person-first language (e.g., obese person).

3. The policy uses weight management terms (e.g., weight loss, lose weight).

4. The policy promotes a diet for weight control.

5. The policy assigns moral values to food choices (e.g., good and bad food, clean eating).

6. The policy promotes physical activity associated with weight loss.

7. The policy uses body mass index (BMI) as an indicator of health status. 


\section{Appendix J}

\section{Messaging Analysis Coding Protocol}

\section{Weight Inclusive Message}

Text

1. The recommended behavior is one that would be encouraged to people of all sizes.

2. Person-first language is used (e.g., person with obesity instead of obese person).

3. Uses health-focused terms.

4. Promotes flexible, individualized eating based on internal cues of hunger, satiety, pleasure, appetite, and individual nutrition needs.

5. Does not assign moral values to food choices, instead using objective terms (e.g., healthy food, unhealthy food).

6. Promotes life-enhancing physical activity that allow people of all sizes, ability, and interests to engage in enjoyable movement, to the degree they choose.

7. Promotes decoupling health and weight, not using body mass index (BMI) as an indicator of health status.

Images of People

1. Portrays person who is overweight or obese wearing professional clothes.

2. Portrays person who is overweight or obese engaging in physical activity.

3. Portrays person who is overweight or obese as an expert or advocate.

4. Portrays person who is overweight or obese as a full person.

5. Portrays person who is overweight or obese engaging in eating or drinking healthy food or drink.

\section{Weight Stigmatizing Message}

Text

1. The recommended behavior is one that would be encouraged only to people who are overweight or have obesity.

2. Weight management terms are used (e.g., weight loss, lose weight).

3. Words are used to imply a person has to look a certain way (e.g., beach body).

4. Derogatory terms are used to describe a body part (e.g., muffin top, spare tire). 
5. Written content does not use person-first language (e.g., obese person).

6. Weight management terms are used (e.g., weight loss, lose weight).

7. Written content promotes a diet, drug, program, product, or surgery for the primary purpose of weight loss or weight control.

8. Written content assigns moral values to food choices (e.g., good and bad food, clean eating).

9. Written content promotes physical activity associated with weight loss.

10. Body mass index (BMI) is included as an indicator of health status.

Images of People

1. Disproportionately emphasizes the abdomen or lower body of a person who is overweight or obese.

2. Portrays the abdomen without clothes of a person who is overweight or obese.

3. Person who is overweight or obese is not shown as a full person (e.g., only shown from neck down).

4. Portrays person who is overweight or obese with inappropriately fitting clothes (e.g., a shirt that is distinctly too tight).

5. Portrays person who is overweight or obese eating or drinking unhealthy food or drink.

6. Portrays person who is overweight or obese engaging in a sedentary activity (e.g., watching television, playing video game).

7. Before and after weight loss pictures are used. 


\section{Appendix K}

\section{SELF Magazine Style Guide}

\section{Recommendations for Words and Editorial Content}

1. Don't assume that everyone wants to lose weight or change their bodies.

When reporting on weight loss or another form of body modification as a goal, try to couch with "if your goal is " [to lose weight, to have a six-pack, etc.]

2. When reporting on weight loss, diets, body modification, or fitness challenges, make clear that this information is not appropriate for everyone, and that it might be a good idea to consult with a healthcare provider if you have any questions.

As reporters, journalists, and editors, our role is to analyze the available research and interview qualified experts for their best advice, and then synthesize the information in a way that's easy for people to understand. Health isn't a one-size-fits-all thing, and while our reporting is sound and provides a good baseline of general information, it's likely not going to apply to every single person, such as for people with underlying medical conditions, like a history of disordered eating.

3. Only use the terms "overweight" and "obesity" when referring to specific medical conditions in the context of providing public health-related information.

People frequently use "obesity" and "overweight" in stigmatizing and offensive ways. There are unfortunately no other terms that can accurately describe these specific medical conditions or health markers, as defined by body mass index, and used commonly in the scientific literature. When it's possible to use other terms without sacrificing specificity or accuracy in our reporting, we will use those instead. And, though it's significantly less stigmatized, we will also only be using "underweight" in the same medical context.

4. If it's necessary to describe a person's body size or type, use their preferred descriptor if possible. e.g., fat, small fat, curvy, thick, plus size, bigger bodied

5. Avoid derogatory terms to describe body parts. e.g., saddlebags, cankles, bat wings, spare tire, bra bulge, muffin top 
6. Avoid implying that a person has to look a certain way or be a certain size to wear specific clothing or participate in specific activities. e.g., Bikini body, beach body, tank top arms

7. Be specific and accurate when discussing the connection between weight and health. We have an extensive primer on the research; link to it whenever it contextually makes sense.

8. When reporting on eating disorders or disordered eating behavior, adhere to recommendations from the National Eating Disorders Association (NEDA) for responsible media coverage.

9. When reporting on weight loss or other body fat composition goals, do your best to provide thorough context about what goes into losing weight, the cultural forces motivating a person to want to lose weight, and the fact that weight loss can be really hard and maintaining weight loss even harder.

Providing context is crucial: Explain that weight and weight loss are impacted by a variety of factors, including sleep, stress, hormones, medications, other health conditions, access to healthy foods, affordability, and other things that may be outside of someone's control. Provide cultural context when possible as well, especially when discussing a person's motivation to lose weight or change their body shape. Avoid promising or implying that a person can expect specific weight loss or body modification results in a set period of time, or that they can look like a celebrity (or get the body parts of a celebrity) simply by doing specific workout moves or eating specific foods. And make it clear that losing weight or focusing on weight loss isn't right for everyone and can potentially be harmful, particularly for anyone with underlying health conditions, including a history of disordered eating.

10. When reporting on celebrity health, nutrition, or fitness choices, behavior, or statements, consult with actual experts and evidence-based research to provide appropriate context and information.

Celebrities are not health experts. But they are often in the news for the things they say and do related to health, fitness, and nutrition. A lot of people find this stuff interesting and potentially helpful. When reporting on celebrity-related information, always speak with qualified experts and refer to any relevant research. 
Be cautious not to imply that it's possible to look like a celebrity by doing a specific thing that a celebrity does. It's unrealistic and very likely untrue. When reporting on celebrity fitness routines in particular, address this misconception directly and make it clear that's not what you're implying.

A quick note about debunking inaccurate celebrity health advice: We tend to shy away from doing debunkers every time a celebrity says something wrong. For starters, the original bad health information generally spreads significantly faster and farther than debunkers do. Furthermore, there's some concern that repeating the bad information, even in the context of providing facts that prove it wrong, might introduce people to the bad idea in the first place, and/or may actually reinforce belief in the factually incorrect statement. It's not a hard and fast rule, but we generally choose to do debunkers when the story is already really huge and we think that enough people are Googling it or familiar with it.

11. When reporting on weight loss, be clear that there is no "best" diet for weight loss. The general consensus about weight loss and diets shows that there is no one diet or weight loss method that is superior to any other in terms of efficacy. For people who successfully lose weight, the best "diet" is the one that you can actually stick to. For people who successfully are able to maintain weight loss, the key has been a total lifestyle overhaul that they can live with while still enjoying their lives. It's not sexy, it doesn't make good headlines, and it's important that we don't complicate it much more than that. See our primer, So, You Want to Lose Weight and Keep It Off for more information.

12. Avoid reporting on one-off nutrition studies as the basis for a story.

We generally avoid reporting on one-off studies. With nutrition and weight-loss science, that's even more critical—-there's already so much confusing noise out there, and it can result in people having absolutely no idea what healthy eating even means, or how to have a healthy relationship with food.

13. When reporting on “cleanses," "detoxes," weight loss teas, OTC weight loss supplements, and diuretics, be explicit about how those approaches can be harmful and that experts advise strongly against them. Discourage these approaches in unequivocal terms. 
14. Avoid assigning moral value to food choices or demonizing any types of food groups. Some examples: Food isn't good or bad, sugar is not toxic or addictive, GMOs aren't harmful to your health, most people can tolerate gluten just fine, and you aren't misbehaving or deserving of punishment when you eat something high in calories, fat, carbohydrates, sugar, and so on. Avoid the term "clean eating" because it perpetuates this idea that if some food is "clean," the opposite kind of food must be "dirty."

15. When reporting on any type of particular specialty diet, provide necessary context up front about potential risks and sustainability.

You may have noticed that people have very strong opinions about carbohydrates and fat, especially lately (hello, Keto).

We've discussed a lot internally about whether it's OK to report on specific diets, like paleo, intermittent fasting, Keto, low-carb, Whole30, and so on, or provide recipes that are compatible with these diets, given everything we know about weight loss, health, and how dieting is often unsustainable. We've come down on the side that we will continue doing so, for the same reasons that we will continue reporting on weight loss. But in any story referring to those specific diets we should include all relevant contextual information about sustainability and risks, as well as the other information we provide about weight loss as a goal in general. That means we should also include the information that nutrition experts generally discourage the wholesale elimination of entire nutrients or food groups from your diet without consulting with a healthcare professional first. Additionally, when reporting on specific diets, we should point to the evidence that multiple types of diets (for example, low-carb and low-fat) can potentially work for weight loss, and, again, if your goal is weight loss, the best diet is the one that works best for you.

16. When talking about exercise and fitness, avoid framing it in a way that makes it sound like weight loss is the best reason to work out, and avoid positioning exercise as a form of punishment or atonement for caloric consumption.

First, research points to the fact that exercise alone isn't a sufficient method of trying to lose weight. Exercise can contribute to weight loss in that some people who begin 
to exercise will also modify their food intake for a variety of reasons, which can then lead to weight loss. But for many people, exercising can actually cause a slight increase in caloric consumption, which mitigates any extra calories burned while working out. So talking about exercise as if it's going to help someone lose weight, without providing a huge amount of context, is misleading.

More importantly, there are a ton of really good health-related reasons to work out that have nothing to do with weight loss. It's one of the best things most people can do for their health, both mental and physical. We don't want to reinforce the idea that exercise is a chore or something that you have to do as penance or punishment for eating food.

17. When publishing or promoting fitness or nutrition challenges, be explicit about what a participant can and cannot expect to get out of $i$. Challenges should focus on forming healthy habits, or challenging yourself with something new and enjoyable. Fitness and nutrition challenges should not promote weight loss or promise weight loss as a benefit, and we should be clear that - especially with a fitness challenge that doesn't come with a nutrition component—weight loss likely won't happen. All fitness and nutrition challenges should come with clear language indicating that people with underlying medical conditions, particularly a history of disordered eating, should consult with a healthcare professional before participating. (Kylstra, 2018)

\section{Recommendations for Imagery and Packaging}

1. Images or image collections should be diverse and inclusive.

When a picture or editorial package has art with multiple people in it, make the best effort possible to show different types of people, especially with regard to race, body type, size, and ability. For individual stories with one picture, be mindful of the photo selections for stories published shortly before and shortly after, given how they'll appear on the site clustered close together.

2. Avoid editing photos to change the size or shape of a person's body, or to remove cellulite or stretch marks.

It's important to show people as they are, and not perpetuate the idea that a digitally altered body is either ideal or possible to achieve. We will continue to 
edit photos for lighting, styling, and light general clean-up purposes, but photo editing should not be done to change the way a person looks.

3. Avoid publishing before-and-after weight loss pictures.

It's not our place to tell people what pictures they should and should not post of themselves, or that they shouldn't feel proud of themselves for setting a goal and working hard to accomplish it. But as a health media brand, SELF should avoid posting these types of photos. It's important that we do what we can to avoid promoting the idea that there's an ideal body type, that you can tell how healthy someone is just by looking at them, or that the most important thing about a person or their health journey is the way their body looks. Many of the times, these photos can send the opposite message.

4. Avoid graphic pictures of the bodies of people in the midst of an eating disorder. This is one of several NEDA recommendations about how to report responsibly on disordered eating. The images can be extremely triggering to other people with or in recovery from eating disorders. It also sends the message that you can tell if someone has an eating disorder just by looking at them, which isn't true.

5. Avoid photos that graphically or intentionally dramatize thinness. e.g., protruding ribs or pelvic bones

Fetishizing or dramatizing thinness can have public health implications, particularly among people who have eating disorders or are at risk or or in recovery from eating disorders.

You can't tell how healthy or unhealthy a person is by how they look, and we're not policing what kind of bodies are OK to have. We want to portray a range of body types, which can and should include smaller and thinner bodies. This recommendation is meant to guide how to do so thoughtfully.

6. Use pictures of people from marginalized communities in all kinds of stories, not just those stories specifically about that group.

So, for example, don't exclusively use images of people with bigger bodies in stories about living in a bigger body - make imagery for all content as diverse as possible, no matter what the story is about. 
7. Avoid posting images of people from marginalized groups in exclusively negative or sad contexts.

We want to avoid perpetuating the stereotype that people from marginalized groups live difficult, miserable lives - doing so further reinforces stigma that living with this identity is a bad thing. People with bigger bodies, for instance, experience joy, love, pleasure, fun, excitement, success, and happiness, just like people with smaller bodies do, and our creative choices should reflect that reality.

8. With fitness stories, avoid closely cropped pictures of abs, butts, arms, legs, and backs to accompany a corresponding body part workout.

The implication, even if not explicitly stated, is that if you do this workout you'll get abs or a butt that looks like that. It's not accurate or realistic to set an expectation like that. It also unhelpfully conflates exercise with body size or type, which is super oversimplified. And then there's the issue that this type of disembodiment is typically associated with objectification, rather than experiencing someone as a complete human being. We're all more than a sum of our parts. This gets a bit more cerebral than you might expect, considering it's a rule about close-cropped booty pics. Our philosophy is one of bodily autonomy. You can do what you want to do with your body, and if your goal is to have a sixpack or a firm, round butt, that's your call. Our role in this dynamic is to give you evidence-based information about what exactly you would need to do to accomplish that goal, and how to do it in a way that isn't harmful to you, mentally or physically. That means that we should not imply in any way that it's easy to accomplish these goals. And, beyond that, we also need to make clear that for some people (many people, actually), the work required may in fact not be worth the end result, in the sense that it can make you totally miserable and could actually be harmful. And also for some people, no matter how much work you put in, sometimes these physical attributes are genuinely impossible to achieve.

9. Avoid promoting weight loss content adjacent to content about body positivity or disordered eating.

This sends confusing and contradictory messaging. Additionally, there are people who want to engage with our content-particularly the content around body 
positivity and disordered eating — who don't want to see any messaging at all related to weight or weight loss. From an editorial perspective, we should make an effort to avoid this type of overlap or adjacency wherever possible. But this is also a product challenge, in addition to an editorial and visual one-digital infrastructure and UX can impact messaging as well. To that end, we have been working with the product, engineering, and audience development teams to come up with solutions or approaches to addressing these challenges, and will continue to do so.

10. Avoid publishing information about how to lose weight on Snapchat Discover. We have a huge audience on Snapchat Discover. The audience is significantly younger than the audience on other platforms, which makes sense, given that Snapchat has a young user base. Teenagers and young adults are particularly susceptible to messaging about dieting and weight loss, and also at high risk for developing eating disorders. We should continue to avoid publishing any weight loss content to this platform. (Kylstra, 2018) 
VITA

Hillary Roberts was born and raised in Knob Noster, Missouri. She earned a Bachelor of Science in Dietetics degree from Missouri State University in May 2002. She then completed a Dietetic Internship Graduate Program at Iowa State University in December 2002 and became credentialed as a Registered Dietitian Nutritionist. Hillary continued her education, traveling to Sydney, Australia where she completed a Master of Science in Health Science degree from The University of Sydney.

Hillary began her professional career working as a dietitian at a behavioral health hospital for children and adolescents in Springfield, Missouri. She started her career at Missouri State University in August 2004 as an Instructor in the Biomedical Sciences Department, Dietetics Program. During her time at Missouri State, she spearheaded the development of two graduate programs, the Dietetic Internship Graduate Certificate Program and the Master of Science in Nutrition and Dietetics Program. She currently serves as program director for these graduate programs. Hillary began the University of Missouri's Statewide Cooperative Doctor of Education Program in 2017. This education gave her the qualifications to apply for and accept an Assistant Professor position at Missouri State University in August 2018. Hillary and her husband, Brandon, have a daughter Clara and look forward to welcoming baby Mara to the family in April 2021. 